University of Redlands

\title{
Optimizing Oracle Centrography
}

\author{
A Major Individual Project submitted in partial satisfaction of the requirements \\ for the degree of Master of Science in Geographic Information Systems \\ by \\ Travis Conrad Val \\ Douglas Flewelling, Ph.D., Committee Chair \\ Fang Ren, Ph.D.
}

January 2014 


\section{Optimizing Oracle Centrography}

Copyright (C) 2014

by

Travis Conrad Val 
The report of Travis Conrad Val is approved.

Fang Ren, Ph.D.

Douglas Flewelling, Ph.D., Committee Chair

January 2014 
The report of Travis Conrad Val is approved.
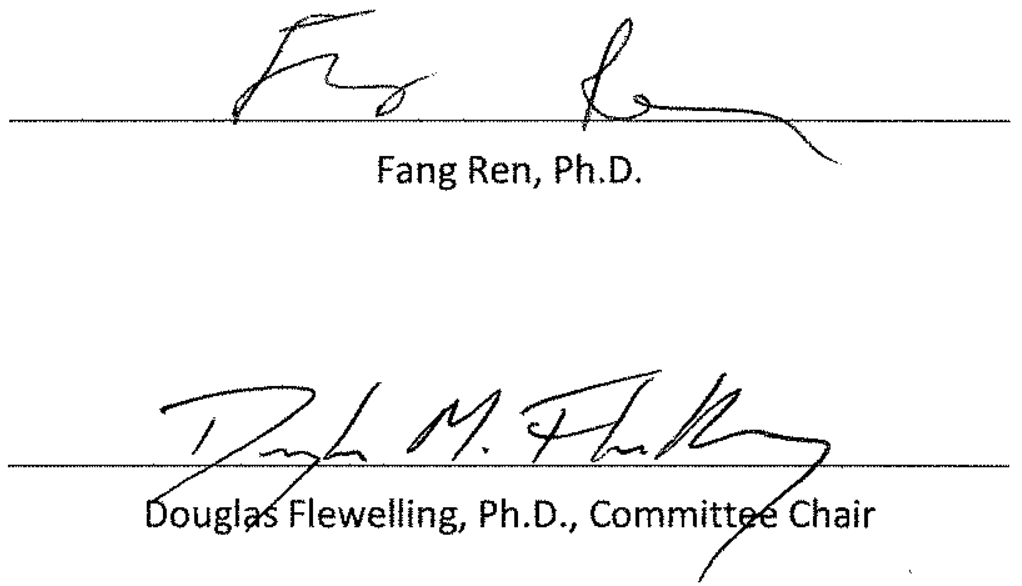

January 2014 



\section{Acknowledgements}

I would like to thank my wife Heather. Her understanding and patience during the last couple of years has been amazing. The support and understanding of my managers and coworkers at Esri has been steadfast. Without this, finishing this program would not have been possible. Much of my success is credited to the staff at the MS GIS program, through their help and guidance. Finally, I thank Professor Fred Wilhelm from the University of California Riverside, who helped me work through some of the more interesting mathematical problems I encountered, even though none of them wound up in the final product. 



\author{
Abstract \\ Optimizing Oracle Centrography \\ by \\ Travis Conrad Val
}

Large datasets pose unique problems for certain statistical operations. This is caused by the requirement for each row to be evaluated one or more times in order to generate the result. In the situation where the client application is on a separate system, or not utilizing metadata to its full potential, this requirement may lead to degradation in performance and efficiency. By generating the results of data intensive operations at the source and returning only the result, these performance problems can be eliminated. Creation of a statistical metadata index on these datasets further increases efficiency by caching partial results for multiple clients to access. This project implements an Oracle package that will employ a user defined domain index with associated descriptive operators and functions for point populations. Using these packages, statistical information can be generated more efficiently for large spatial point datasets stored in Oracle databases, or more quickly using estimates and confidence intervals. 



\section{Table of Contents}

Chapter 1 - Introduction ................................................................................... 1

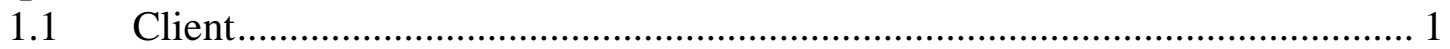

$1.2 \quad$ Problem Statement .......................................................................... 2

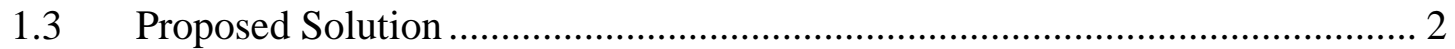

1.3.1 Goals and Objectives ............................................................................. 3

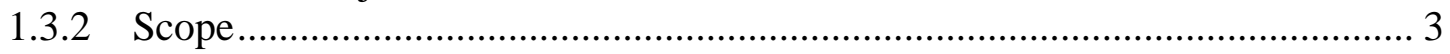

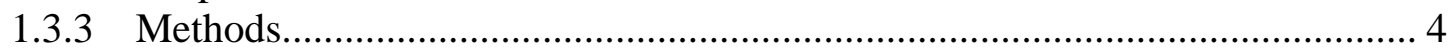

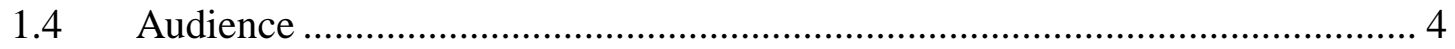

$1.5 \quad$ Overview of the Rest of This Report ........................................................... 4

Chapter 2 - Background and Literature Review ............................................................ 7

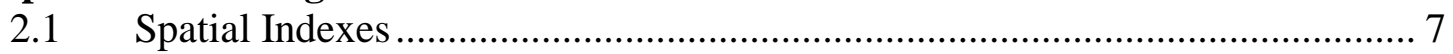

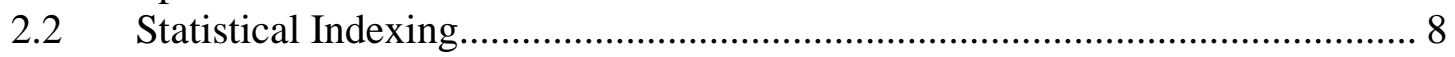

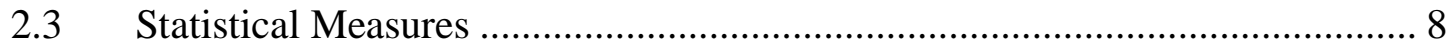

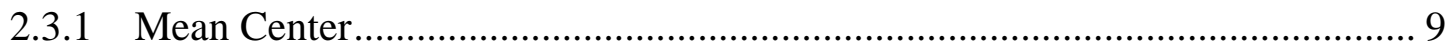

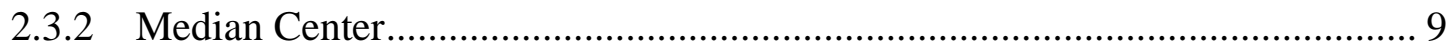

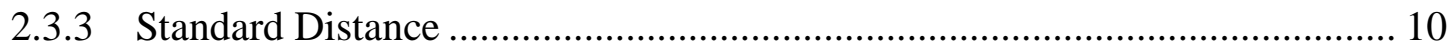

2.3.4 Standard Deviation Ellipse ...................................................................... 10

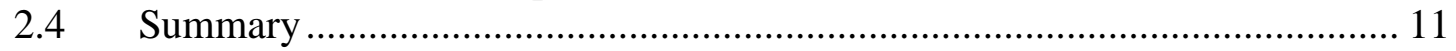

Chapter 3 - Systems Analysis and Design....................................................................... 13

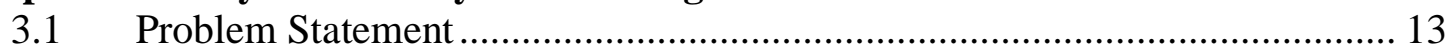

3.2 Requirements Analysis …………………….......................................... 13

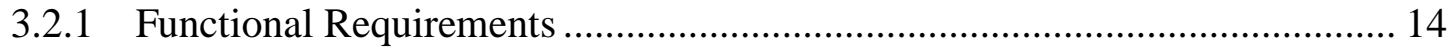

3.2.2 Technical Requirements ........................................................................ 14

3.2.3 Operational Requirements ......................................................................... 14

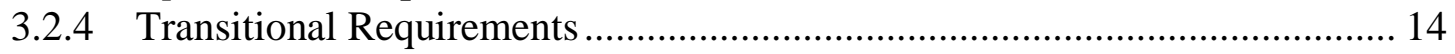

$3.3 \quad$ System Design .................................................................................... 15

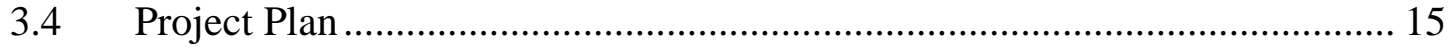

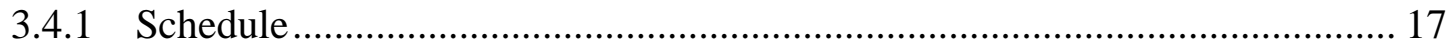

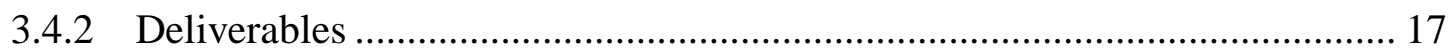

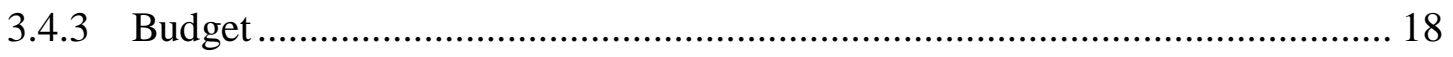

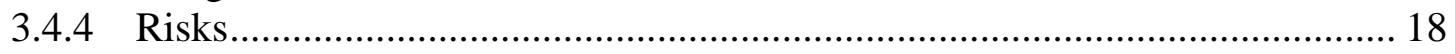

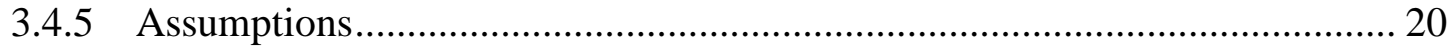

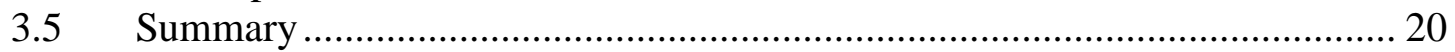

Chapter 4 - System Design ......................................................................................................... 21

4.1 Conceptual Data Model ...................................................................... 21

4.1.1 Exact Mode ........................................................................................ 21

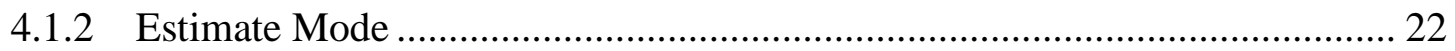

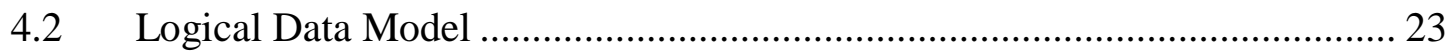

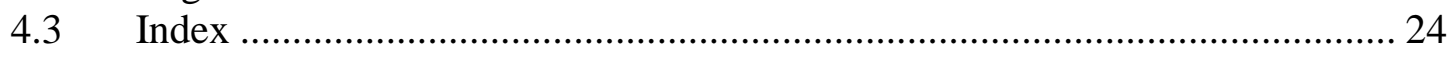

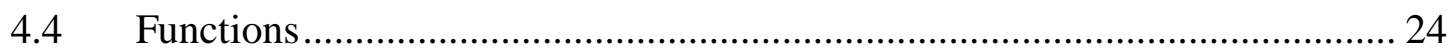

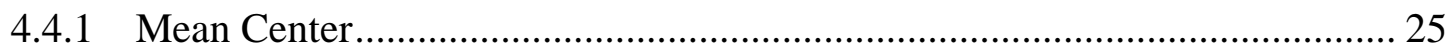

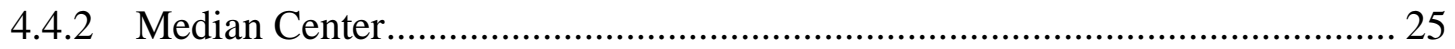




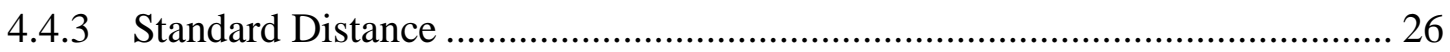

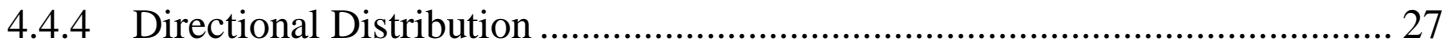

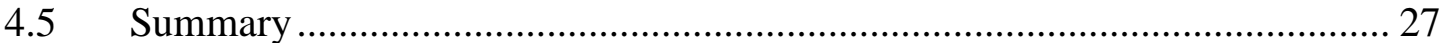

Chapter 5 - Implementation........................................................................................ 29

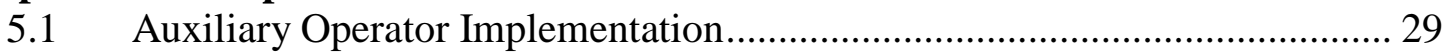

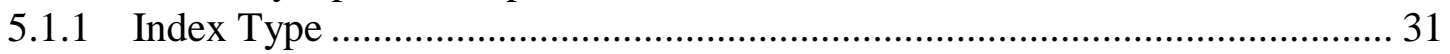

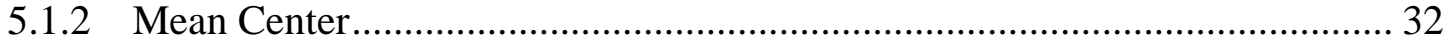

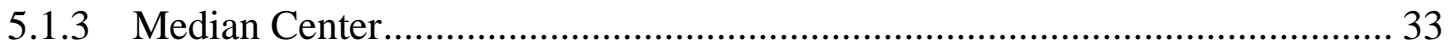

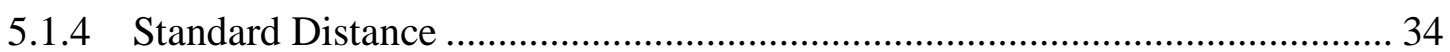

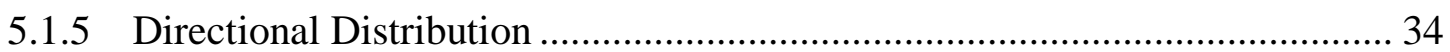

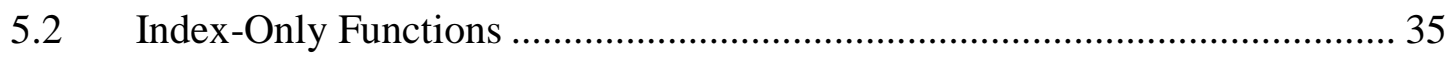

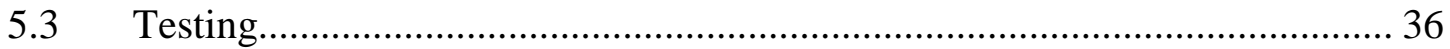

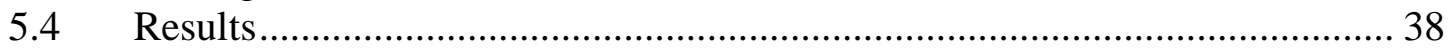

Chapter 6 - Results and Analysis..................................................................... 41

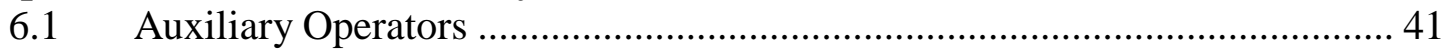

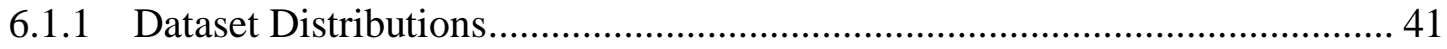

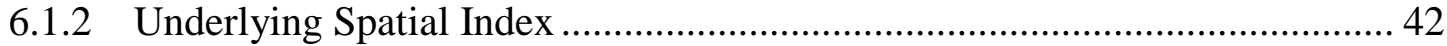

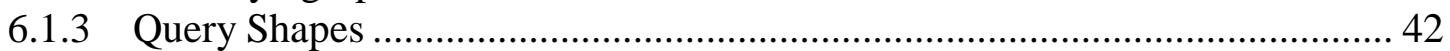

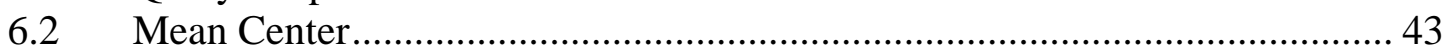

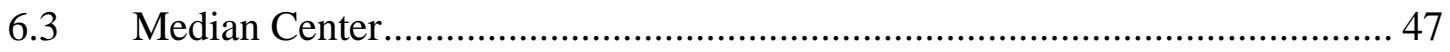

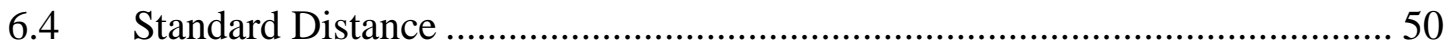

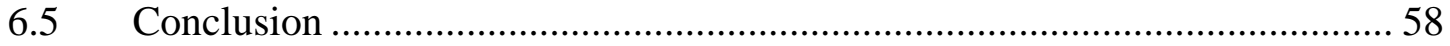

Chapter 7 - Conclusions and Future Work ..........................................................59

Works Cited 61

Appendix A. Files......................................................................................................6 63

Appendix B. Operators ...................................................................................................... 67

Appendix C. Performance Metrics.................................................................................. 72

Appendix D. Test Data .............................................................................................................. 74

Appendix E. Additional Figures................................................................................... 75

Appendix F. User Permissions .................................................................................... 83

Appendix G. Equation Manipulation........................................................... 84 


\section{Table of Figures}

Figure 2-1. Maximum number of grid cells that would have to be evaluated............ 7

Figure 3-1. Project workflow for creating an Oracle statistics package .................... 16

Figure 3-2. Project timeline for creating an Oracle statistics package .......................17

Figure 4-2. The size of the query shape in relation to the size of the grid cells ........22

Figure 4-1. A UML diagram of the systems logical model ...................................23

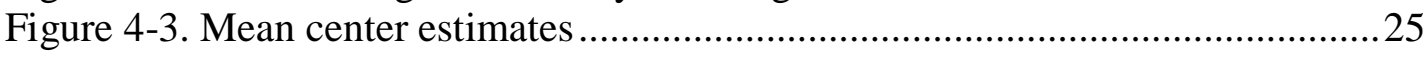

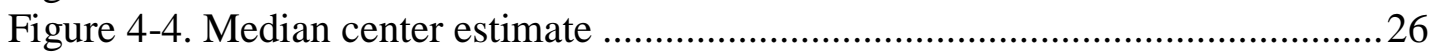

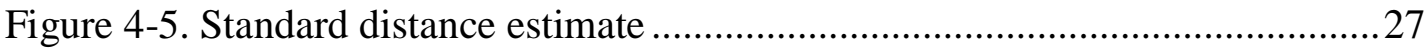

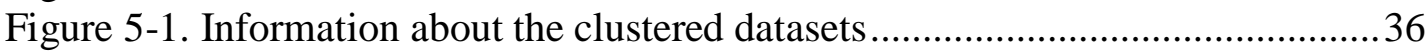

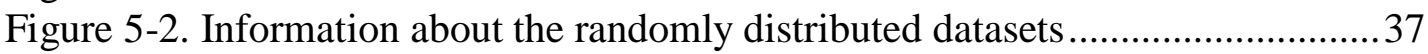

Figure 5-3. Information about the uniformly distributed datasets ..............................38

Figure 6-1. Performance data for mean center calculations on uniform data............43

Figure 6-2. Performance data for mean center calculations on clustered data ..........44

Figure 6-3. Performance data for mean center calculations on random data.............44

Figure 6-4. The variation between the estimated and actual mean center values......45

Figure 6-5. The ST_Mean value and error estimates..........................................46

Figure 6-6. Performance data for median center calculation on uniform data ..........47

Figure 6-7. Performance data for median center calculation on clustered data.........48

Figure 6-8. Performance data for median center calculation on random data...........48

Figure 6-9. The ST_Median value and error estimates..........................................49

Figure 6-10. Performance data for standard distance on uniform data.....................50

Figure 6-11. Performance data for standard distance on clustered data. ...................51

Figure 6-12. Performance data for standard distance on random data. .....................51

Figure 6-13. Error estimates of standard distance against a square query shape and a

uniform distribution ..................................................................................54

Figure 6-14. Error estimates of standard distance against a square query shape and

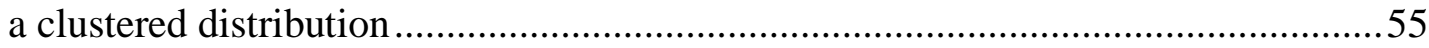

Figure 6-15. Error estimates of standard distance against the USA query shape and

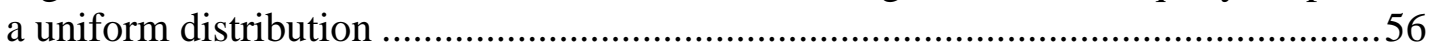

Figure 6-16. Error estimates of standard distance against the USA query shape and

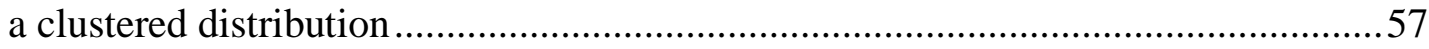





\section{List of Tables}

Table 3-1. Project Tasks and Hours of Effort ............................................................... 18

Table 3-2. Major Project Risks, Severity, Probability, and Exposure ............................ 19

Table 5-1. The public functions and their uses in the ST_STAT_INDEX type............... 31

Table 5-2. The auxiliary operators implemented using functions from the

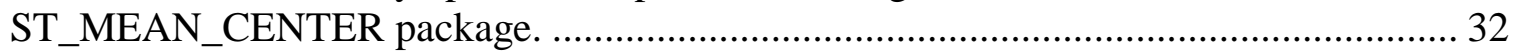

Table 5-3. The auxiliary operators implemented using functions from the ST_MEDIAN_CENTER package.

Table 5-4. The auxiliary operators implemented using functions from the ST_STD_DIST

package.

Table 5-1: The auxiliary operators implemented using functions from the ST_DIR_DIST

package.

Table 5-6. A listing of all operators and functions that had their performance data recorded.

Table 5-7. The listing of data metrics used when analyzing the performance of this solution.

Table 6-1. The cost of the recursive and non-recursive portions of Auxiliary and Aggregate mean center operations 42

Table 6-2. Number of interior and boundary grid cells, and the average execution time using the Estimate or Solve approaches.

Table 6-3. Value, estimate, and error bounds for standard distance operations. 



\section{Table of Code Listings}

Code Listing 4-1: Index create statement. .................................................................. 24

Code Listing 5-1: The difference between index and table results................................. 30 



\section{List of Acronyms and Definitions}

DBMS Database Management System

SQL $\quad$ Structured Query Language

PL/SQL Procedural Language/Structured Query Language 



\section{Chapter 1 - Introduction}

The amount of data being collected is increasing. Social media sources such as Twitter and Facebook generate staggering amounts of data, and the amount of spatial information in these data sources is growing. Adding a picture to Facebook will tag it with a nearby city. Checking in may broadcast the user's exact location. A traditional relational database can process these data, but these processes can require very long run times. Hardware solutions to this problem, such as Netezza, Terradata, and Oracle's Excetdata (Hammergren \& Simon, 2009), work by splitting these data across multiple hardware devices, allowing each device to process a subset of the data. This is very effective, but also very expensive. Three year cost estimates for these systems range from 2 to 13 million dollars (International Technology Group, 2011; Mohanty, 2012; Klopp, 2012). Without the advantage of expensive hardware, is it possible to leverage existing data structures to increase the efficiency of processing, specifically spatial data processing? This project explored whether it is possible to leverage a spatial index in order to decrease the amount of work that a database has to perform to calculate, or even estimate, the mean center of a set of points.

The majority of current software implementations require that statistical calculations be rerun from the start, reading every member of the population one at a time. For large populations, this could be very time consuming and result in a considerable lag between the final data store update and the generation of the statistical results. By tracking changes in the population, the changes can be applied to the model results. This could allow for faster result generation with little to no accuracy loss. This advantageous reuse of previously computed information - coupled with optimized data processing and transmission techniques - allows normally computationally intensive statistical information to be calculated and transmitted to client applications in a fraction of the time conventional implementations require.

This project achieved this efficient statistical package by creating server-side processing packages and database indexing that augment any Oracle system with the Esri SDE.ST_Geometry type installed. Once in place, this augmented system was able to produce accurate spatial statistical results more quickly than the conventional means. The packages calculate the following measures: mean center, median center, and standard distance. A fourth measure - directional distribution — was attempted but ultimately was incompatible with a single pass approach. The inclusion of the three successfully implemented functions increased the profile of the SDE.ST_Geometry type in the user community, as well as increasing the functionality available to users. This new functionality allows them to gain better and faster insights into their data.

\subsection{Client}

The client for this project was the ArcSDE team at Esri. This team manages the interaction of Esri software with enterprise-level databases such as Oracle, SQL Server, and Postgres SQL. Their main goals for this project were to demonstrate the flexibility and extensibility of spatial types, and to increase the standing of the SDE.ST_Geometry type in the user community. The point of contact for this project was Forrest Jones, the 
ArcSDE for Oracle testing lead. Kevin Watt, an ArcSDE developer, agreed to support this project in a strictly technical aspect. He had no administrative responsibilities, but made himself available for technical support during the implementation of this project.

\subsection{Problem Statement}

Currently, to automatically perform spatial statistical analysis requires a software package with statistical functionality, such as ArcGIS (ESRI, 2011aa) or the use of Oracle aggregate operators (Oracle, 2010b). Software packages that participate in a client/server type of architecture have two different system designs: one where the processing is done on the server and one where the processing is done by the client application. Typically, client applications must be installed on the client machine for the application to function. This client software is often developed in such a way that it can work with a variety of data storage formats, types, and schemas, leading to greater flexibility in the consumption of data. The client application's structure and flexibility in accessing these different data formats leads to limits in its ability to perform spatial analysis in two ways. First, the client software must access the data. This usually involves reading the data from the data server and transferring them across some sort of network. Second, because the client must interact with a variety of data formats, specifics about each format must be ignored. Information like spatial indexes cannot be exploited directly because that information may not be consistent across all the different supported data formats. As statistical operations are very data intensive, performance of these tasks may be decreased by failing to use metadata such as spatial indexes, database level functionality, and server-side hardware capabilities that are designed to operate on very large datasets. Implementing these statistical functions at the database level allows the database functionality and structures designed for this type of data to be exploited for increased performance. It also does not require the transmission of the data to the client machine.

The functions that this project implements were designed in a similar fashion to Oracle's current statistical functionality, requiring minimal changes to applications that currently consume them. The project concentrated on functions designed to measure geographic distributions of point datasets, although there were a number of different problem types that could have been addressed, including nearest neighbor calculations, spatial pattern analysis, and grid-based system simulations.

\subsection{Proposed Solution}

The solution to this project had two main parts, including the components that users would employ to generate statistical information. These included the functions, operators, and indexes that exist in the Oracle instance and return the statistical information. These include mean center, standard distance, directional distribution, and

median center. Section 4.4 details the execution of these operators. The second part is a method that evaluated the functions, operators, and indexes for accuracy and efficiency. Using this testing component to quantify any changes in performance or accuracy is one of the measures by which to gauge the success of this project. 


\subsubsection{Goals and Objectives}

In a very general sense, the client had two goals for this project: to increase the reputation of the SDE.ST_Geometry spatial type on Oracle in the user community, and to improve the user experience. To this end, this project aimed to increase the spatial type's functionality by adding a statistical framework capable of operating on very large datasets. This was implemented through the creation of user-defined objects in an Oracle database that use Oracle's Data Cartridge interface.

More specifically, the first objective was to include a statistical package at the database level that offered statistical functionality to any user connected to the system. The second objective of the project was to include an automatic method of storing information that had already been computed by one user for any other user to access. This would greatly increase the system's efficiency. The proposed solution for this project addressed these objectives, implemented as a set of packages and a testing program to evaluate the accuracy and efficiency of any of the newly created functions.

To be considered a success, the functions that generate the statistical information had to outperform both client and server-side (aggregate) functions that perform similar operations. It also had to satisfy the client's goals of increasing the status of the geometry type and improving the user experience.

\subsubsection{Scope}

The implementation this project involved significant amounts of PL/SQL programming. This included the implementation of the index structures, the contains operator, and the mean center, median center, standard distance, and directional distribution functions. These functions had to perform Cartesian calculations on projected data. Earth centered XYZ calculations on geodetic (non-projected) data were valuable components to this, but were ultimately beyond the scope of this project. All statistical calculations operated in three modes: a full scan mode that returns the exact result; an estimate mode that returns a fast estimate along with upper and lower values that bound the true result; and an automatic method that switches between the modes when certain conditions are satisfied. The project also included the method to evaluate the results of the functions in order to quantify any change in performance or accuracy.

The data that fell within the scope of this project was limited to 12 datasets; one for each of the combinations of four different sizes - approximately 6000, 60,000, 600,000 and 6,000,000 points - and three different distributions: random, uniform, and clustered.

This project did not address any optimizer statistics. Furthermore, the resultant packages, operators, functions and any other deliverable were provided to the client at final delivery. Once the client took final delivery of the material, all responsibility for maintenance, version changes, bug fixes or other problems became their responsibility.

The client responsibilities were restricted to technical assistance in the design and implementation phases of the project. This was limited to advice and support in the design and implementation of the first component. During the design phase, this included guidelines for what were acceptable differences - precision, accuracy, 
efficiency — in results and runtime between the ArcGIS geoprocessing tools and the implemented packages.

\subsubsection{Methods}

To achieve this project's goals, a method of pre-aggregating values from the underlying population into regularly spaced bins was implemented. These bins coincided with the grid cells of the spatial index. Each of these bins stored information about the underlying population that can be combined in order to satisfy the requesting function. For example, in the case of mean center, the relevant bins would be located and the values stored in these bins would be aggregated in a way that produces the sum of $\mathrm{X}$, sum of $\mathrm{Y}$, and a count of all points. This is all the information needed to generate the mean center of that point population. The key to this method is that data contained by the bins are all that is needed for a continuous area. These bins are stored in an optimized table, an Index Organized Table. This means that instead of having to read a large number of rows from the base table, a smaller number of rows can be read from the index table more quickly.

This logic was built into the packages and types that implement the Oracle Data Cartridge. This is the structure that Oracle provides in order to facilitate user-defined functions, operators, and indexes, also known as domain indices. The index is used to ensure that the bin information is kept current, while the operator filters the incoming data. The functions recombine the filtered information in a variety of ways to return the correct result.

The effectiveness of these functions was measured by testing a number of different geometric shapes against different sizes of datasets. In each case, the results were compared to the current method of accessing the result, and changes in accuracy, time and, disk I/O were used to gauge the successfulness of the results.

\subsection{Audience}

There are two main target audiences for this report. The first is any party who may not have exposure to database concepts and spatial statistical measures. This could include managers, geographers, or programmers who would like to utilize the functions created by this project. These groups are the target audience for this chapter, as well as chapters 2, 3, and 7. Chapters 4, 5, and 6 are targeted at an audience that is better versed in database theory and spatial statistical measures. Examples of this group include database developers, spatial developers, and spatial systems architects. Chapter Four is targeted more towards the database audience, while Chapter Five is targeted at the spatial statistics audience.

\subsection{Overview of the Rest of This Report}

The remainder of the report outlines this project in detail. Chapter 2 contains the literature review regarding the issues related to this project as well as a discussion of the differences between definitions of median center, approaches to indexing, and other spatial indexing methods that were found. Chapter 3 includes the bulk of the analysis and planning of the project, such as information regarding projected timelines, risks and 
deliverables. Chapter 4 outlines the database and package design. This includes a discussion on the Oracle Data Cartridge and its limitations, the design of the implemented packages, the methods used to generate the test datasets, and the methods and the design of the testing harness. Chapter 5 outlines the implementation and the problems that were faced and an in depth discussion on the overall function operation including the methods used to generate the true result bounds. Chapter 6 discusses the results of the functions on each of the different datasets, using different query shapes. This chapter is an attempt to estimate the behavior of the functions given different types of data. Finally, Chapter 7 outlines this project's conclusions and the future work that may be worthwhile. 



\section{Chapter 2 - Background and Literature Review}

The majority of relational database management systems depend on some sort of indexing to speed the access to data. In the Oracle Database case, not only can indexes be used to speed access to individual rows, they also are used to satisfy the results of a query. For example, querying the minimum or maximum value from a column with an index can result in Oracle's query optimizer accessing the index's first or last block. This means that the database does not actually read the results from the table but from the index. This is a subtle difference that results in more efficient and faster access to data. Similarly, there have been a number of approaches to access spatial statistical information in the same way. Section 2.1 summarizes the spatial indexing approach. Subsequent sections (2.2 and 2.3) summarize statistical indexes and the statistical measures respectively.

\subsection{Spatial Indexes}

Oracle Database documentation describes an index as being an optional structure associated with a table that can sometimes speed data access (2010aa). Indexes do this by allowing random access to records that would normally take a full table scan to access. There is no guarantee that the presence of an index will aid in complex analysis of data. Operations performed on the data must execute in a way that incorporates the strengths and avoids the weaknesses of the index to achieve maximum performance. The Esri SDE.ST_Geometry spatial type employs a fixed grid spatial index (Esri, 2011b). This means that if the search radius is limited to half the grid size as a rectangular range, any search will have to evaluate at most $2^{\mathrm{d}}$ grid cells, where $\mathrm{d}$ is the number of dimensions in the index (Samet, 2006) (Figure 2-1).

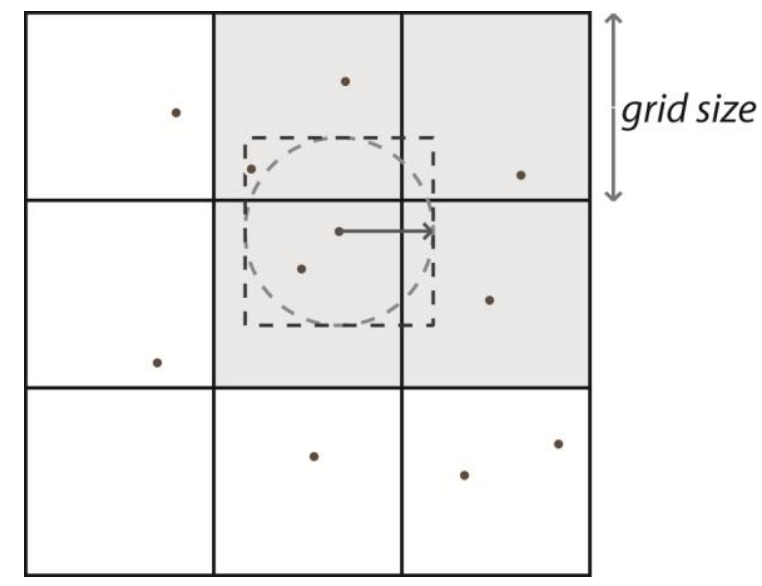

Figure 2-1. The maximum number of grid cells that would have to be evaluated with a search radius of one-half the grid size. This is a representation of a two-dimensional (d) surface, resulting in $2^{\mathrm{d}}$, or $\mathbf{4}$ grid cells. 
Since SDE.ST_Geometry indexes only support $\mathrm{x}$ and $\mathrm{y}$ dimensions, this will be at most four grid cells. Ignoring factors like these may lead to performing a greater amount of work for a relatively small amount of additional data. Conversely, if the majority of a table needs to be read, accessing it through the index would be inefficient. Reading the whole table from start to finish, also known as a full scan, would certainly satisfy the query without having to read the index structure.

\subsection{Statistical Indexing}

Traditionally, a full scan of the data is required to determine spatial patterns. Generally, the calculation of statistical measures, such as mean center, considers all points as required by the equations and processes defining these metrics. Meaning that these equations and processes requires the system to evaluate all locations at least once, limiting the efficiency that these operations can achieve. Wang et al. (1997) proposed a different approach where the generation of statistical information for a point distribution is stored in a grid structure and used later to answer statistical questions about that dataset by evaluating that question on a cell by cell basis. The authors referred to this approach as a statistical information grid-based method, or STING. This method determines what areas are likely to include certain types of values, based on statistical distributions. For example, given a dataset of all household incomes, the STING method can generate results for queries based on household density, income range, location, and total area based upon a confidence level.

\subsection{Statistical Measures}

Statistical information has long been applied to simplify and communicate geographic information. Whether this is done effectively and honestly is up to the geographer doing the work. Well-composed maps and graphics can communicate information very well, but misleading maps and graphics may lead to infamy (Huff, 1954). In a particular incident, a bank produced a map that was colored in such a way to mislead the public on government spending. Although this is a single example, it is still important to be aware that this behavior is possible. Without a proper understanding of the methods used to generate and display datasets, cartographers and graphic designers may accidently create misleading information, and worse, have others make decisions based on statistical misrepresentation.

This is true for both centrography and cartography. In both cases, large amounts of data are distilled into much smaller sets of information. This leads to the loss of data for the sake of comprehension. Creating a national choropleth map displaying state populations loses information about county, census block or track populations, but it is easier to interpret. Similarly, calculating the state's population's mean center loses data on the distributions of people in the state, but it is easier to interpret than displaying the population of every census block or track in the country. The remainder of this chapter will concentrate on the processes by which this statistical aggregation is performed. 


\subsubsection{Mean Center}

The mean center (Equation 2.1) is similar to the mean of a set of numbers (Rogerson, 2006). That is, the sum of the different dimensions is calculated and then divided by the number of members of the population.

$$
\bar{x}=\frac{\sum_{i=1}^{n} x_{i}}{n} ; \quad \bar{y}=\frac{\sum_{i=1}^{n} y_{i}}{n} ; \quad \bar{z}=\frac{\sum_{i=1}^{n} z_{i}}{n}
$$

In essence, the mean center can be thought of as the point where the distance squared between that point and every other point is at a minimum. Equations 2.2, 2.3, and 2.4 convert polar coordinates - north pole equals zero degrees latitude $(\theta)$ with longitude $(\varphi)$ - to a three dimensional space. These three equations substituted into Equation 2.1 illustrate properties of the mean center statistic on the surface of a sphere (Fisher, Lewis, \& Embleton, 1987).

$$
\begin{gathered}
x=\sin \theta * \cos \varphi \\
y=\sin \theta * \sin \varphi \\
z=\cos \theta
\end{gathered}
$$

In this case, except for very small areas, the mean center will be positioned somewhere below the surface of the sphere (Fisher, Lewis, \& Embleton, 1987, p. 32). These equations vary depending on how the axis and angles are defined. For geographers, a more appropriate set of equations are listed by Kumler and Goodchild (1992) as equations 2.5, 2.6, and 2.7. These are the dimensions that are based on equatorial angles (equator is 0 degrees latitude), not polar angles.

$$
\begin{gathered}
x=\cos (\text { lat }) * \cos (\text { lon }) \\
y=\sin (\text { lat }) \\
z=\cos (\text { lat }) * \sin (\text { lon })
\end{gathered}
$$

As with the mean of a population, this measure does not provide any context about the distribution of the point.

\subsubsection{Median Center}

The median center is a point that is located where the sum of the distances from that point to all other points is minimized (O'Sullivan \& Unwin, 2010). This differs from the mean center, where the sum of the squared distances is minimized. Solutions for more advanced forms of this problem are described by Brimberg, Hansen, Mladenović, \& Taillard (2000). These solutions show best results when heuristic approaches are used that correspond to Voronoi partitions in the dataset. Simpler versions of the problem 
have iterative solutions but can incur significant costs when applied to large datasets (Coope, 1963).

A second definition of median center is one used by Kumler (1992). The definition is one that mirrors the median of a non-spatial population; a point that bisects the population into two halves in each of the population's dimensions. If this is performed on a sphere, there may be many different points and orientations that satisfy this solution. Specifying that the point's orientation follows the lines of latitude and longitude reduces the solution to at most two points, one for each side of the sphere. Placing an additional constraint that this is calculated using a spatial index means that the sphere is projected onto a plane. This reduces the possible outcome to one point.

\subsubsection{Standard Distance}

The standard distance of a population (Equation 2.8) is a measure of the variance of a population. It is defined as the square root of the average squared distance (Rogerson, 2006). This is similar to the definition of standard deviation in a population of numbers.

$$
S_{d}=\sqrt{\frac{\sum_{i=1}^{n}\left(d_{i c}\right)^{2}}{n}}
$$

Where $d_{i c}$ is the distance from the current point to the mean center. This dependency on the mean center requires two full scans of the dataset, one to calculate the mean center and one to calculate the standard distance. Rearranging this equation into another form (Equation 2.9) allows for a single pass of the data.

$$
s_{d}=\sqrt{\frac{\sum_{i=1}^{n} x_{i}{ }^{2}+\sum_{i=1}^{n} y_{i}^{2}}{n}-\bar{x}^{2}-\bar{y}^{2}}
$$

Combining the mean center and standard distance, it is possible to quantify the center and distribution of a population. This roughly illustrates the amount of distribution of a dataset, but gives no indication on the distributions of the different dimensions.

\subsubsection{Standard Deviation Ellipse}

Separating the axes and allowing them to rotate to the angles of highest and lowest variation yields the standard deviation ellipse, or directional distribution. This method works because the standard deviation along any axis is actually a function of the standard deviation along any other axis (Lefever, 1926). This allows the minimum and maximum values to be calculated, and as they are always perpendicular to each other, they are suitable for the definition of an ellipse. The equations put forth by Lefever (1926) are based upon the standard distance equation (Equation 2.8) and later refined by Yuill (1971) (Equations 2.10 and 2.11).

$$
s_{d x}=\sqrt{\frac{\sum_{i=1}^{n}\left(x_{i}-\bar{x}\right)^{2}}{n}}
$$




$$
s_{d y}=\sqrt{\frac{\sum_{i=1}^{n}\left(y_{i}-\bar{y}\right)^{2}}{n}}
$$

If the rotation to the angle of lowest and highest variation is included, the distance between points must be corrected to the new axis. Equations 10 and 11 become Equations 12 and 13, where $\theta$ (the angle of greatest variation) is defined as Equation 14 through 17.

$$
\begin{gathered}
s_{d x}=\sqrt{\frac{\sum_{i=1}^{n}\left(\left(x_{i}-\bar{x}\right) \cos \theta-\left(y_{i}-\bar{y}\right) \sin \theta\right)^{2}}{n}}, \\
s_{d y}=\sqrt{\frac{\sum_{i=1}^{n}\left(\left(x_{i}-\bar{x}\right) \sin \theta-\left(y_{i}-\bar{y}\right) \cos \theta\right)^{2}}{n}} \\
\tan \theta=\frac{A+B}{C} \\
A=\sum_{i=1}^{n}(x-\bar{x})^{2}-\sum_{i=1}^{n}(y-\bar{y})^{2} \\
B=\sqrt{(A)^{2}+4 \sum_{i=1}^{n}((x-\bar{x})(y-\bar{y}))^{2}} \\
C=2 \sum_{i=1}^{n}((x-\bar{x})(y-\bar{y}))
\end{gathered}
$$

Although the standard deviation ellipse does display more information than any of the previously mentioned statistics, it does not always represent the actual data distribution. Furfey (1927) points out that in certain situations, the standard deviational ellipse proposed by Lefever (1926) can be misleading, such as if the population is scattered along a line or non-symmetrically about the mean. To display all populations accurately another method, such as a raster grid or iso-lines, must be employed.

\subsection{Summary}

As Furfey pointed out with the standard distance ellipse, all of these statistical measures carry with them some sort of simplification or aggregation of data. Geographers have a responsibility to employ them correctly to communicate the underlying dataset accurately. The goal of this project was not to propose new measures, but to increase the efficiency of the current measures, thereby increasing the understanding of the spatial phenomena. 



\section{Chapter 3 - Systems Analysis and Design}

Large datasets pose specific problems for data intensive operations. This project proposed and implemented a method of increasing the scalability of certain statistical measures by using pre-computed data stored in a database index. This indeximplemented with the Esri SDE.ST_Geometry spatial type_-facilitates four statistical measures: mean center, median center, standard distance, and directional distribution. This project was started in January 2010, with a projected completion in December 2011, but was actually completed in December of 2012. Variations from this original timeline and deliverables are outlined in Chapter 4.

\subsection{Problem Statement}

Statistical measures require the consideration of every point in the population. If client applications process the data, all the data must be transmitted to the client. A more efficient model would process the data at the source, returning only the result to the client. This optimization, although significant, may not be enough to satisfy the client.

Two common techniques to choose from for server side processing of large datasets are either simultaneous evaluation of many partitions of the dataset, or to have some portion of the result cached to decrease the amount computation required to fulfill a request. The former method involves increasing the amount of hardware available; the latter involves creating structures that can access a subset of the data more quickly. This project implemented the latter. Specifically, this project implemented a method to compute information about point membership in a grid index, and the associated functions that have the ability to consume that cached data.

\subsection{Requirements Analysis}

The implemented statistical functions must outperform current client-server based implementations. The proposed system achieves this by implementing functions that operate on point populations and indexes that calculate and cache results. As a result, the functions use pre-computed values and do not need data for the entire population.

As different databases and database types have different index structures, and the close relationship of the implemented functions to the underlying index, the statistical package is dependent on the SDE.ST_Geometry Type. The index that this system creates does not degrade over time, but due to the previously mentioned dependency, it must reflect any changes made to the underlying index. Any additional load that this will cause the servers would normally be spent at non-peak times, such as overnight, allowing normal operations to continue uninterrupted. Transitioning from a currently implemented client based statistical system can be achieved without interruption, as the systems are not mutually exclusive to a client-based system that employs SDE.ST_Geometry as a storage type. 


\subsubsection{Functional Requirements}

The implemented functions must produce accurate answers for the statistical metrics previously listed. These metrics are the basis for much more complex measures, but are the most data intensive. Therefore, increasing the efficiency of these base matrices will increase the efficiency overall. The complexity of the SQL interface is not a concern, as hand written SQL statements are not the intended method of interaction with this or any other SQL interface. Typically, a client application with spatial or statistical abilities will access these SQL functions. The client applications act as the end user interface, so complexity in this project's information products is acceptable.

\subsubsection{Technical Requirements}

This project does not implement spatial indexing and spatial relationships, and provided functionality is inconsistent between databases. Therefore, the statistical functions implemented by this project depend on an Oracle database that has the SDE.ST_Geometry spatial type installed and enabled. Any SDE.ST_Geometry column should also have a valid SDE.ST_Spatial_Index built on it to facilitate optimal performance. Due to the number of spatial relationship tests that must be performed in order to maintain and resolve the statistical questions that are required, any increase in performance that can be achieved through tightly coupling the metadata index and spatial indexes are extremely beneficial. Unfortunately, this also results in a dependency on the SDE.ST_Geometry spatial index and utility functions.

\subsubsection{Operational Requirements}

Over time, the metadata index will not degrade. There is no need to rebuild the index regularly, like other tree-based indexes. However, the metadata index is heavily dependent on the spatial index and that index's structure. For this reason, if the underlying spatial index is rebuilt, so must the metadata index. System operations will actually improve due to a decrease in long-term load on the database server provided by the new statistical operators. The operations that increase server load, such as rebuilding indexes, can be scheduled at times of low server usage.

\subsubsection{Transitional Requirements}

Implementing a new system that utilizes these functions will require no transition. Transitioning a currently implemented system from a current statistical system to the system implemented by this project can be performed in a manner that allows the use of both the current and new systems. This is possible because the systems are not mutually exclusive as long as both systems utilize the SDE.ST_Geometry spatial type. If the current system does not utilize the spatial type, then the migration of that system to SDE.ST_Geometry type would have to be accomplished before the statistical system migration can occur. 


\subsection{System Design}

The system was implemented in two main parts, the index, and the operators. The index maintains the consistency of the aggregated (cached) data and uses those data to provide the operators with the necessary information to calculate the required metric. These operators calculate the mean center, median center, standard distance, or directional distribution.

More specifically, the index maintains a separate table containing aggregated data that is combined to generate each of the statistical metrics, without the need to read the underlying base table. This leads to performance gains as access speeds and data density on this index table are much higher than the base table. When a query is issued, the initialization routines of the statistical index call methods on the underlying spatial index, generating information about what data are relevant to the query shape. It then reads this data, again aggregating them into different population values. These values - such as the sum of all $x$ values, the sum of all $y$ values, and the number of points - are the values that the operators use to generate the actual statistical metrics. In this case, dividing the sum of all $x$ values by the count of values will yield the $x$ portion of the mean center. The operators are responsible for generating the actual measures and any error estimates that are requested. A technical description of all index and operator functionality is available in the next chapter of this report, along with discussion on the conceptual and logical models of this system and specific details about the implementation of the index and operators.

\subsection{Project Plan}

The solution was implemented using the main tasks outlined in Figure 3-1, separated into design, implementation, verification, and completion phases. Of these four phases, the design phase was the most significant. The key component of this phase was to finalize all the design decisions for the proposed packages and index. For instance, at the end of this phase what functions should be implemented, what median center calculation to use, and questions about the statistical index structure had been finalized. The implementation phase was straight forward after making all the major decisions during the design phase. The verification phase was the most difficult and the one in which the amount of time was underestimated by roughly a year. In this phase, a number of expected and unexpected problems were encountered and remedied, including limitations in the Oracle Data Cartridge, PL/SQL and the initial design of the project. 


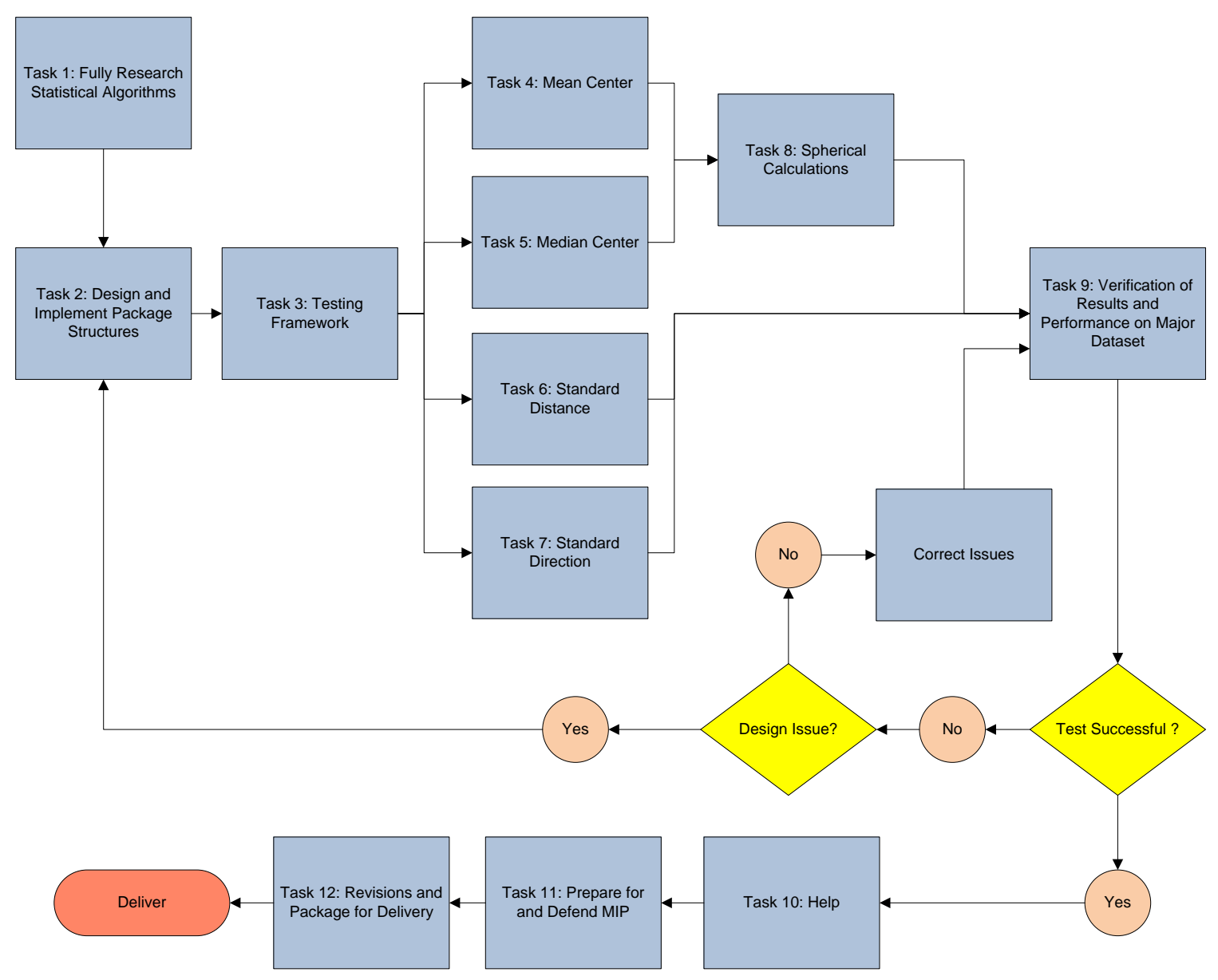

Figure 3-1. Project workflow for creating an Oracle statistics package

During the design phase of the project (Tasks 1-2), the goal was to research and design the Oracle packages. At the end of this phase, the design was set and accounted for all expected functions, operators, relationships, and tables. Ensuring that all of these components compiled and were valid before continuing was critical, as it is easier to fix design issues earlier in the development process. Encountering a design issue later in the project would require a redesign and possibly a reimplementation of the entire project.

The implementation phase started with the creation of the testing framework (Task 3 ), followed by the implementation of each of the functions (Tasks 4-8). The objective was to implement a function, then test that function using small 6000 row datasets. When the testing was unsuccessful, the problem was corrected and the function was retested. This was repeated until testing was successful, at which point that function was considered complete and the process started over with the next function.

The next phase of the project was the verification phase. This was where more rigorous testing was performed on each of the functions. Testing the functions using a larger $(600,000-6,000,000$ row) dataset (Task 9) was performed. This was the final phase of testing before delivery, so it had to be exhaustive. As the dataset size was much bigger, this required a large amount of unsupervised processing time. Based on original 
estimates, this task was allotted one week. This proved to be an insufficient amount of time. In total, this phase was allotted 60 hours but required more than 250 hours. One of the errors, described in detail in section 5.1.5 of this report, required major changes to the design of the index.

After the implementation was rigorously tested, help documentation was assembled from the design document, Oracle documentation, and the comments within the PL/SQL packages themselves (Task 10). This resulted in multiple documents that include topics on the index, each of the packages, and the functions. These topics outline the methods used in generating results, and best practices associated with each of the topics.

Finally, preparation for the defense, the defense itself (Task 11 and 12) and delivery to the client occurred.

\subsubsection{Schedule}

The schedule for this project is shown below (Figure 3-2). Additionally, there were two deliverables with this project. These deliverables were provided when certain functionality was completed and available for client review. The first phase began April 18 signifying the commencement of the design phase of the project. The next phase, implementation, started on September 5. The verification phase began Oct 31, when the majority of the implementation was complete. The length of this phase was two weeks, which was a gross under estimation. The final phase began November 14, with completion of the project December 19.

\begin{tabular}{|c|c|c|c|c|c|c|c|c|c|c|c|c|}
\hline Task Name & Jan '11 & Feb'11 & Mar'11 & Apr'11 & May' 11 & Jun '11 & Jul'11 & Aug '11 & Sep'11 & Oct '11 & Nov'11 & Dec'11 \\
\hline \multicolumn{13}{|l|}{ Proposal - GIS691 } \\
\hline \multicolumn{13}{|l|}{ Prgiect - GIS692 } \\
\hline \multicolumn{13}{|l|}{ Planning - GIS693, GIS694 } \\
\hline \multicolumn{13}{|l|}{ Phase 1: Design } \\
\hline \multicolumn{13}{|l|}{ Task 1: Research } \\
\hline \multicolumn{13}{|c|}{ Task 2: Design and Implement Structure } \\
\hline \multicolumn{13}{|c|}{ Task 3: Testing Framework } \\
\hline \multicolumn{13}{|l|}{ Phase 2: Implementation } \\
\hline \multicolumn{13}{|l|}{ Task 4: Mean Center } \\
\hline \multicolumn{13}{|l|}{ Task 5: Median Certer } \\
\hline \multicolumn{13}{|l|}{ Task 8: Spherical Calculations } \\
\hline \multicolumn{13}{|c|}{ Task 6: Implement Standard Distance } \\
\hline \multicolumn{13}{|c|}{ Task 7: Implement Directional Distribution } \\
\hline \multicolumn{13}{|c|}{ Phase 3: Verification } \\
\hline \multicolumn{13}{|l|}{ Task 9: Verification of Results } \\
\hline \multicolumn{13}{|l|}{ Correction } \\
\hline \multicolumn{13}{|l|}{ Phase 4: Completion } \\
\hline \multicolumn{13}{|l|}{ Task 10: Help } \\
\hline \multicolumn{13}{|l|}{ Task 11: Prepare and Defend } \\
\hline \multicolumn{13}{|l|}{ Task 12: Package for Delivery } \\
\hline Delivery & & & & & & & & & & & & \\
\hline
\end{tabular}

Figure 3-2. Project timeline for creating an Oracle statistics package

\subsubsection{Deliverables}

The final deliverables for this project were a set of SQL scripts to install the statistical packages, the help documentation, a testing program with installation instructions, and a 
report. In addition to the final deliverables, there were two additional deliverables to the client. These include the project proposal (February 25) and a planning document (August 17). The planning document included all the function signatures and a description of their functionality.

\subsubsection{Budget}

Table 3-1 outlines the budget for this project. The listed hours are estimates of the amount of time required to complete each of the tasks outlined earlier in this chapter.

Table 3-1. Project Tasks and Hours of Effort

\begin{tabular}{l|ll}
\hline Task & Description & Hours \\
\hline 1 & Fully Research Statistical Algorithms & 60 \\
2 & Design and Implement Package Structures & 60 \\
3 & Testing Framework & 20 \\
4 & Mean Center & 40 \\
5 & Median Center & 40 \\
6 & Standard Distance & 20 \\
7 & Directional Distance & 40 \\
8 & Spherical Calculations & 60 \\
9 & Verification of Results and Performance on & 60 \\
10 & Major Dataset & 15 \\
11 & Help & 40 \\
12 & Prepare for and Defend MIP & 20 \\
& Revisions and Package for Delivery & 475 \\
\hline
\end{tabular}

\subsubsection{Risks}

Some significant obstacles had to be resolved before this project could be completed, the main one being unfamiliarity with the Oracle domain index and Data Cartridge structures. Other minor obstacles included the implementation of a large number of complex components and the need to demonstrate added value to the SDE.ST_Geometry type.

Technically, the only major obstacle was that the design of this project had to be based on the Oracle Data Cartridge framework. This framework needed to be studied in order to create and implement a successful design. Other possible obstacles, including statistical and spherical algorithm research, were minor because they are well documented and only have a direct effect on certain components of the project. Failure to resolve any of these may have been unfortunate and lead to a change in the project scope, but would not prevent the completion of the project.

Economically, this project's feasibility was based on time management. Due to the number of different components in this project, each component had to be completed 
within the allotted time. If the component was not completed on time, and additional time was spent trying to accomplish that task, other components were put at risk.

Organizationally, the client had to accept that this project adds value to the SDE.ST_Geometry type. As the client was involved at a technical level during all phases of this project, they had many opportunities to raise concerns regarding this risk and propose solutions to it.

Table 3-2. Major Project Risks, Severity, Probability, and Exposure

\begin{tabular}{|c|c|c|c|c|}
\hline Risk & Severity & Probability & Mitigation & Exposure \\
\hline $\begin{array}{l}\text { Time } \\
\text { management }\end{array}$ & 3 & 4 & $\begin{array}{l}\text { Strictly follow timeline } \\
\text { and milestone goals }\end{array}$ & 12 \\
\hline Design & 5 & 1 & $\begin{array}{ll}\text { - } & \text { Rigorous design } \\
\text { - } & \text { Research index and } \\
\text { optimizer structure } \\
\text { - } & \text { Implement prototyped } \\
\text { system to ensure } \\
\text { functionality is } \\
\text { possible }\end{array}$ & 5 \\
\hline Accuracy & 1 & 5 & $\begin{array}{l}\text { Regular testing of the } \\
\text { results against a } \\
\text { baseline standard } \\
\text { - } \quad \text { Correct error when } \\
\text { they are found } \\
\text { - } \quad \text { Accurate bug tracking }\end{array}$ & 5 \\
\hline Efficiency & 2 & 2 & $\begin{array}{l}\text { - Regular testing of the } \\
\text { performance of the } \\
\text { packages } \\
\text { - Design for } \\
\text { performance from the } \\
\text { start }\end{array}$ & 4 \\
\hline
\end{tabular}

Overall, the two main risks for this project were time management and design. Other risks included accuracy and efficiency. Time management was critical in this project to minimize the risk of spending too much time on a single component of the project. The risk of errors during the design of the project held a high amount of exposure, although the risk was low. If there was an error in the design, the only way to correct that error may have been to redesign the entire system. A rigorous design document can minimize the risk of this problem developing, but if it does develop, the redesign of the project would be a major task. The risk of accuracy problems was very high, but these can be detected and easily corrected using a testing framework. Efficiency issues can also occur, but proper programming techniques and attention to efficient processes during the creation of the packages minimized these. 


\subsubsection{Assumptions}

This proposal made one major assumption: that it is possible to create an index that behaves in the required manner. Traditionally, index behavior is to return a row from the underlying table. In this case, the index needs to return pre-aggregated information, not the rows themselves.

\subsection{Summary}

Although this project was far behind schedule, the majority of the time estimates were reasonably accurate. The verification phase was vastly underestimated due to unknown limitations in the PL/SQL programming language. This is discussed more extensively in section 5.1.1. Additionally, the only major assumption that this project made was incorrect. While it is possible to replicate some functionality that standard Oracle functions provide, it is not possible to reproduce it all. More specifically, it is not possible to implement an index aware aggregate function. Functions, auxiliary operators and aggregate functions can be implemented, but they only provide a portion of the assumed behavior. Sections 5.1 and 5.2 discuss this in more depth. 


\section{Chapter 4 - System Design}

Information about the design of the system is contained within this chapter. This includes the conceptual and logical models followed by the index and function designs. The actual implementation of the index and functions had to be done using the structures that Oracle provides. The method that Oracle provides for extending indexing and defining these objects is the Oracle Data Cartridge. By defining types with specific member functions and defining those types as index types, operators, or functions, Oracle will use them when and as they are needed. For example, once implemented, defined, and created, indexes defined in this way are fully supported with optimizer statistics, such as costs and cardinality used to generate execution plans. Implementing systems in this framework allows not only for leveraging features that Oracle has provided, but also for prospective users to easily understand the access structure of the system. As the design of the index and functions must be implemented within the Oracle Data Cartridge, this will affects the conceptual and logical models.

\subsection{Conceptual Data Model}

The index and the four operators - mean center, median center, standard distance, and directional distribution - comprise the majority of the solution. The index maintains the consistency of the metadata and the generation of the aggregate values, while the operators recombine those aggregate values to produce the required results.

The index is more aptly described as a metadata index. It maintains information about each grid cell in the underlying spatial index. The grids are defined by the underlying spatial index. The statistical index accesses this information to generate lists of candidate cells that are then used by the operators to generate the aggregated results from the data stored in the metadata index. When an edit occurs, the statistical index either adds or subtracts the appropriate values from each of the values in the index table to maintain consistency with the underlying dataset.

The operators combine the information from the statistical index to produce results. Each operator does this differently, but each operates in one of two modes: exact or estimate mode.

\subsubsection{Exact Mode}

Exact mode produces an exact result. This involves finding all grid cells that are within the geometry and aggregating their values. The boundary cells are iterated, and any point that lies within one of these cells is tested for intersection with the query geometry. The points that intersect the query geometry are then added to the interior cell aggregates. Although this returns an exact result and there are no error estimates, it does involve iterating the boundary cells and testing each individual point for intersection with the query shape. This is time consuming and may require large amounts of data to be stored in memory to produce a solution. 


\subsubsection{Estimate Mode}

In the estimate mode, the underlying points are never considered directly; each interior cell is counted and a result is generated. This ignores the boundary cells, assuming that the addition of the boundary cells have an insignificant effect on the result. This is due to the increasing ratio of the size of a contiguous shape in comparison to the size of an index gird. As a result, the number of interior cells increases at a rate that is greater than the rate at which the number of boundary cells increases. The rate of increase is dependent on the geometry of the shape, but in general, the internal area of a shape will increase exponentially, while the perimeter of the shape will grow linearly. When this ratio is small, the addition of the relatively few boundary cells has little to no effect on the result.

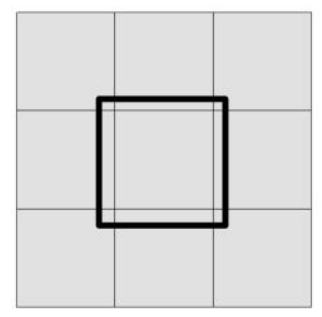

Interior Grid Cells: 1

Boundary Grid Cells: 8

Total Grid Cells: 9

Interior / Boundary Ratio: 1/8 $=0.125$

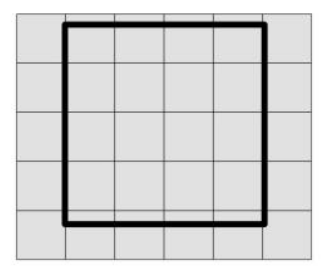

Interior Grid Cells: 12

Boundary Grid Cells: 18

Total Grid Cells: 30

Interior / Boundary Ratio: 12/18 0.667

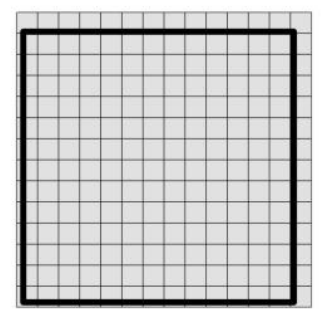

Interior Grid Cells: 144

Boundary Grid Cells: 52

Total Grid Cells: 196

Interior / Boundary Ratio: 144 / $52=2.769$

\section{Figure 4-2. As the size of the query shape grow in relation to the size of the grid cells, the ratio of interior to boundary cells increase.}

As accurate as this method may be for situations where this interior-to-boundary ratio is very small, the results will always have some error present. It is possible to estimate the upper limit of the error by recalculating the measure for each of the different extreme positions by including a subset of the boundary cells and recalculating the measure. Since accessing the information about which points intersect the query geometry is expensive, the entire cell is counted and the error is considered the maximum error that is possible. Any boundary cell with a value greater than the estimate will serve to pull the estimate in the positive direction. Similarly, any cell with a value less than the estimate will serve to pull the value in the negative direction. For mean and median center, the boundary cells are used to calculate the error values in each of the four 
directions: positive, negative, $\mathrm{X}$, and $\mathrm{Y}$. Any boundary cell that is in the same row or column as the estimate is ignored. This does omit some data, but again, the effect of this relatively small amount of data should be negligible. For standard distance, the boundary cells that are closer than the standard distance to the mean center count towards the negative error, while cells that are greater than the standard distance are counted toward the positive error. Any cell that intersects the standard distance is ignored. This produces three concentric circles, with the middle one being the estimate. There are no error calculations made for the standard direction.

\subsection{Logical Data Model}

The statistical index and operators are dependent on the presence of the SDE.ST_SPATIALINDEX. This index defines the grid cells that must be used by the statistical index in the aggregation of statistical information. Each of these cells is known as a STAT_CELL and has a number of values associated with it. They are:

- Sum of $x$ and sum of $y$ for mean center

- Sum of $x$ squared and sum of y squared for standard distance

- Sum of $x$ squared times $y$, sum of y squared times $x$, and sum of $x$ squared times y squared for directional distribution

These same values are also reflected in the index type, and represent the values that will be used by the operators to calculate their respective statistical measures. While the statistical index is initializing, these values are aggregated to generate overall totals for interior and boundary values. The interior and boundary cell membership and point counts are also aggregated for use in the median center calculations.

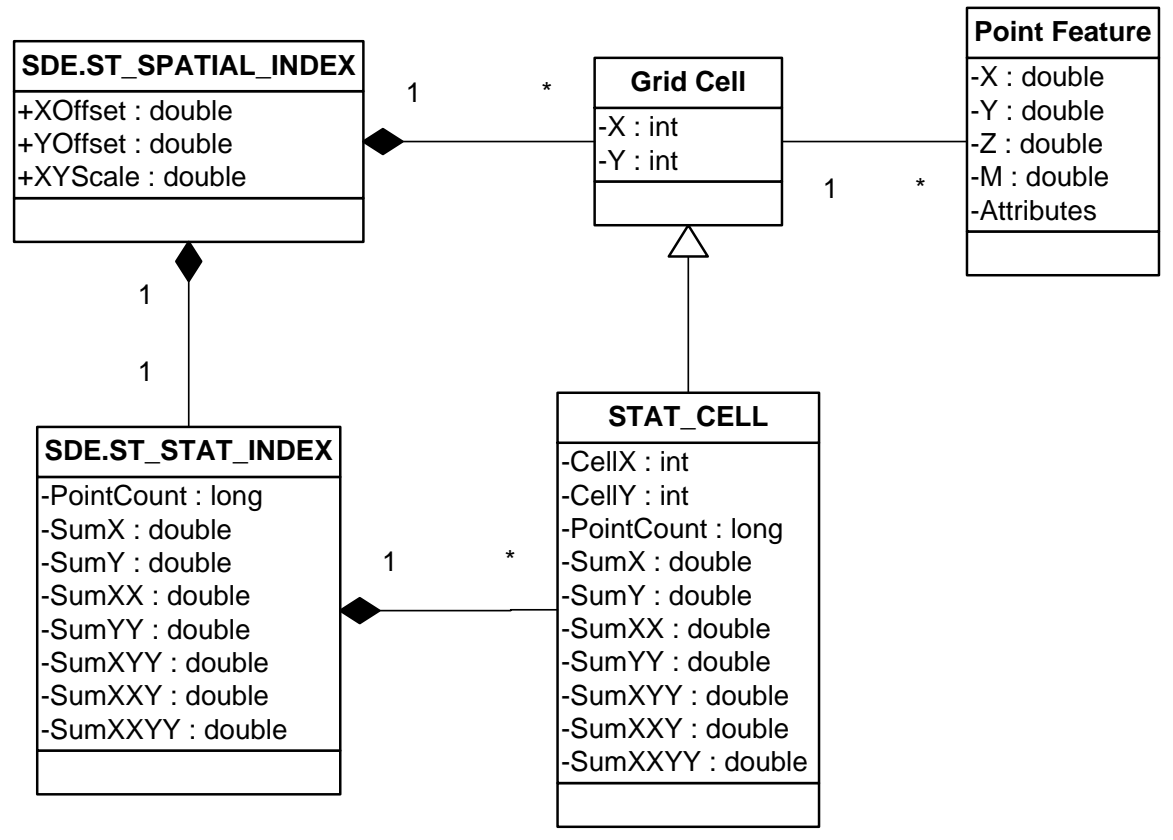

Figure 4-1. A UML diagram of the systems logical model. Each of the elements is dependent on the existence of a spatial index. 
To estimate the error associated with a particular estimate, the data in the statistical index need to be regrouped. For mean center, this includes the sum of x, sum of y, and point count for boundary cell, grouped by row and column. Median center requires no additional information to generate estimates, but does require lists of interior points that are contained within boundary cells. Standard distance error calculations require the sum of $x$ squared and sum of y-squared values to be recorded for each boundary cell. All this information is either read or generated during index initialization.

\subsection{Index}

The index itself is a domain index defined using the Oracle Data Cartridge. It is created using the statement found in code listing 4-1.

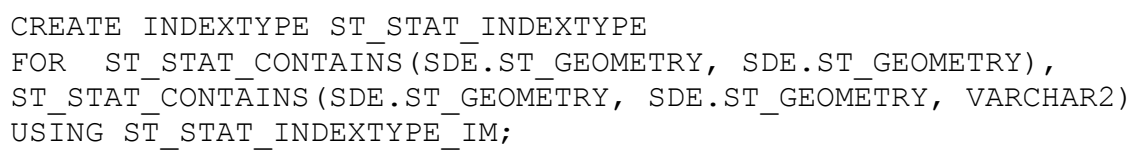

\section{Code Listing 4-1: The index create statement with two versions of the contains operator.}

There are two operators that will invoke this index when used in a where clause. ST STAT CONTAINS (SDE.ST_GEOMETRY, SDE.ST_GEOMETRY) and ST_STAT_CONTAINS (SDE.ST_GEOMETRY, SDE.ST_GEOMETRY, VARCHAR2). In both these cases, the SDE.ST CONTAINS operator is invoked on the underlying spatial index to determine the spatial relationship between each of the points and the query shape. In this case, as with all Oracle operators, the indexed (point) column must be placed first, with the query shape (polygon) placed second. This statement uses the ST_STAT_INDEXTYPE_IM type to define the behavior of the index. This is the Oracle type that is passed to the statistical operators, more correctly named auxiliary operators, and contains all the statistical index context information. The overloaded ST_STAT_CONTAINS operator is used to define the type of operation that will take place, using either EXACT or ESTIMATE inputs. Not specifying a type of operation will result in the index choosing to estimate the value if the interior to boundary cell ratio is greater than a configurable value name IBRATIO; otherwise, an exact result is generated. More research is needed to determine a suitable value for IBRATIO, but in this project's testing a value of 100 was used to cause the $6 \mathrm{~K}$ and $60 \mathrm{~K}$ datasets to use EXACT and the $600 \mathrm{~K}$ and $6000 \mathrm{~K}$ datasets to use ESTIMATE.

\subsection{Functions}

The four functions that were implemented by this project are mean center, median center, standard distance, and standard direction. Each of these functions is dependent on the statistical index. The operators are correctly called auxiliary operators, as they have the 
index context passed to them during their execution. In this way, any information that the index calculates is available to the functions.

\subsubsection{Mean Center}

As previously discussed in chapter 2, the mean center is calculated using Equation 2.1 in section 2.3.1 of this report. This is done by entering the values from the spatial index and solving the equation for each of the different dimensions. In the case of an exact solution this results in an exact value, with the error values set to zero. In the case of the estimate solutions, only the interior cells are evaluated. To estimate the maximum possible mean center values, the boundary cells are iterated and each value of the sum of $\mathrm{x}$ and sum of $\mathrm{y}$ are added to the interior values, either increasing or decreasing along the given axis. This will lead to two pairs of values:

- The sum of interior $x$ values, plus the sum of boundary $x$ values from cells with a column number greater than the column that contains the estimated value

- The sum of interior $x$ values, plus the sum of boundary $x$ values from cells with a columns number less than the column that contains the estimated value

Similar values are calculated for $y$-axes. These values are then divided by their respective point counts to yield the estimated maximum mean center values, as illustrated in Figure 4-3.

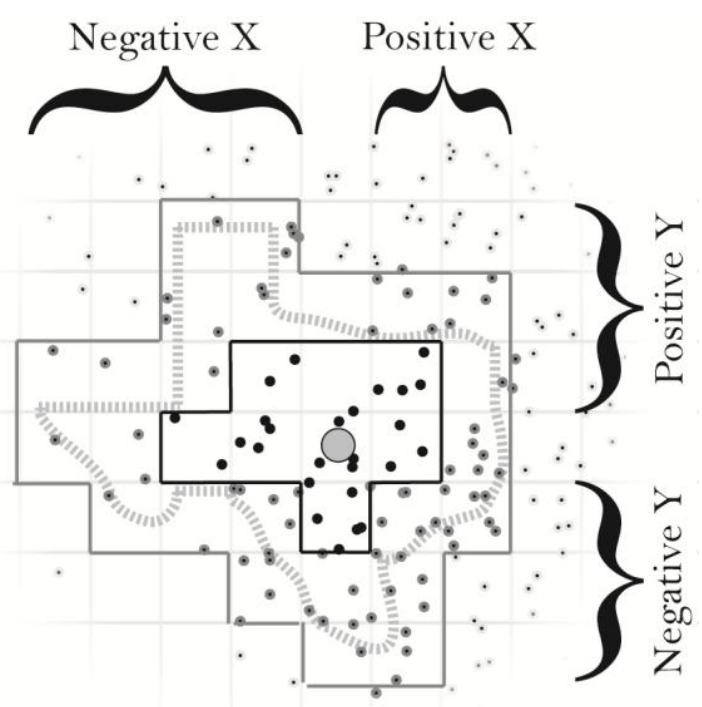

Figure 4-3. Mean Center. The mean center estimate of a population is produced by estimating the result using the sum of $x$, sum of $y$ and count values of the interior cells (outlined in black). Using the boundary cells (outlined in grey) an estimate for each of the four directions is produced.

\subsubsection{Median Center}

Median center is the middle point of the population or, in a population with an even number of points, the average of the middle two. In order to generate this metric, the 
index counts the number of points in each row and column. The median value in each direction can be found by calculating the ordinal position of the median point, determining which column or row contains that point and then sorting the points in that particular row or column . By first finding the column or row that contains the required point, the number of points that need to be sorted is reduced, increasing the process's efficiency.

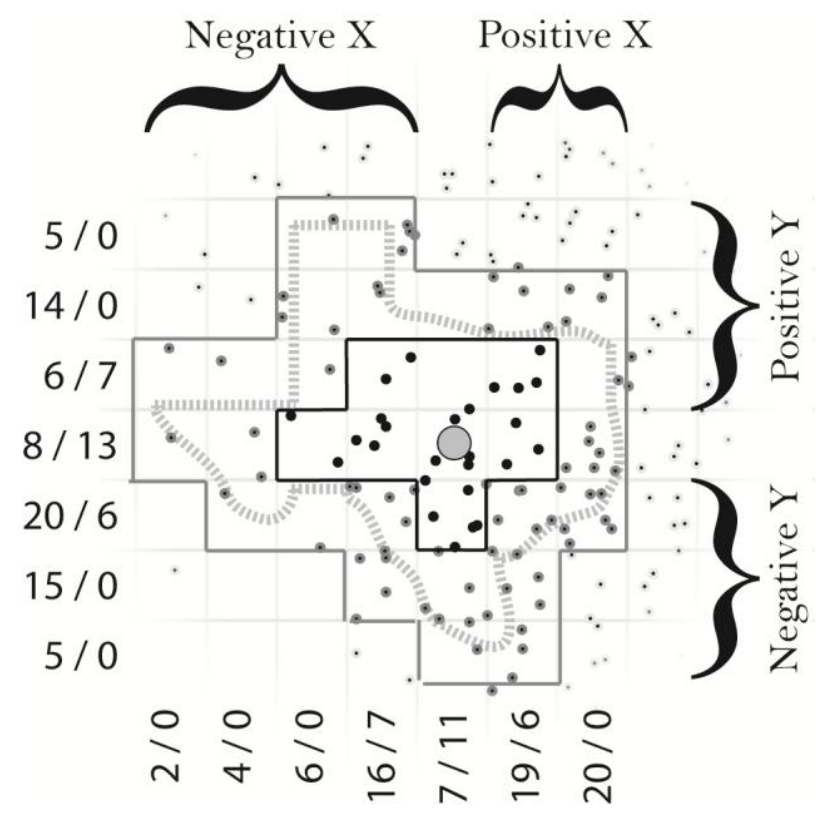

Figure 4-4. The median center estimate of a population is produced by estimating the result using point counts in the interior cells (outlined in black, second number), then using the cells boundary cells (outlined in grey, first number) to produce the error estimates.

In this case, the spatial index table is used to access the result rather than the base table, as the spatial index table is more efficient than the underlying base table, and the statistical index table does not store this information.

Similarly to the methods used in the calculation of mean center, for the exact solution all points are considered, but for the estimated solution only the points contained within interior cells are counted. The maximum median center values are generated by including those boundary cells that are included in columns or rows greater than or less than the column and rows the estimated value is contained by. These new point counts are then used to find the median center's ordinal position in a specific column and row.

\subsubsection{Standard Distance}

The standard distance is calculated according to Equation 2.9 in section 2.3.4 of this report. In the case of an estimated solution, the error values produced create three concentric circles. As in the mean and median center error generation, the estimate of standard distance includes only the interior cells. To calculate the minimum and maximum error, each boundary cells is iterated. Any cell that falls wholly within a circle 
centered at the estimated mean center with a radius of the standard distance is counted towards the negative error. Any cell that falls wholly outside this circle contributes to the positive error. Any cell that intersects the line is not counted.

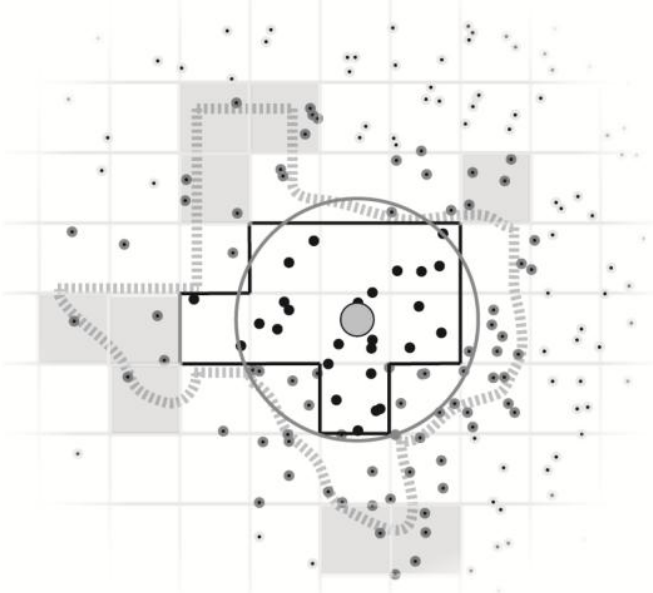

Figure 4-5. The standard distance, represented here by the circular grey line, is calculated by the cells outlined in black. The error is calculated by the cells shaded grey. These cells contribute to the positive error of the estimate; no cells in this case contribute to the negative error.

\subsubsection{Directional Distribution}

Directional distribution is outlined in Section 2.3.4 of this report. No suitable method was found in order to quantify the amount of error in the calculation of $\theta$ (the angle of maximum variation), so the estimated solution will return the directional distribution of all the interior cells. No estimate values are available. This feature accounts for about half the width of the statistical index table.

\subsection{Summary}

The two different solution modes are key concepts to this solution. In each case, the statistical index and operators attempt to balance accuracy with performance in order to yield the fastest, most accurate estimates possible. This is done through the use of the spatial and statistical index tables, as well as pre-computing information when possible. This solution also attempts to return results without estimation and loss of accuracy, but as the ratio between shape size and grid cell size increases, the additional cost of an exact solution begins to outweigh accuracy gain. 



\section{Chapter 5 - Implementation}

This project was implemented using a text editor for PL/SQL programming and Visual Studio 2010 to create and management the testing framework. The original design of the operator and functions failed because it was not possible to achieve the specified functionality with the operator types provided by Oracle. To work around this issue, auxiliary operators were used to implement the proposed system. Section 5.1 of this report details this work, along with several issues that were encountered. A different solution to this limitation would be to create a function that consumes the spatial index directly. Section 5.2 details how this structure would work, and why this option was not used. The remainder of this chapter will detail the implementation of the testing framework that was created, followed by a description of the results. Results discussion can be found in Chapter 6.

\subsection{Auxiliary Operator Implementation}

The original idea for implementing these functions was one similar to the current Oracle provided aggregate functions, such as MIN, MAX or COUNT. These aggregate functions are index aware, although the exact method used to implement these aggregates has not been published. The aggregate functions, if used correctly, have the ability to access indexes built on the column they are querying and return the correct result from the index rather than the table. Figure 5-1 shows the different Oracle Explain Plans (the method by which the DBMS resolved the query) generated by a query that uses the MIN aggregate function, with and without the use of an index. The built-in Oracle aggregate functions are extremely versatile, as they will aggregate in the absence of an index or when the where clause prevents the index from being used. When implementing userdefined aggregate functions, this type of behavior is not possible because the index context is not provided to the aggregate functions. The auxiliary operator method was used, as it is closest to this behavior. In this case, the index context is passed to the function when the function executes. This is very powerful, as information calculated by the index is available to the operators. In the case of this project, all the information that is needed by the operators is calculated by the index and provided to the operators. The operator combines these data to return the value requested by the SQL statement.

These operators are more effective than simple aggregate functions, but they have some major limitations which stem from the Oracle Data Cartridge, the framework that all user-defined indices and operators must use. The index must return a row from the underlying table and the framework does not provide the SQL statement where clause of the user's query. The index must return rows from the underlying table. This is what indexes typically do. However, during the execution of an aggregate function, the resultant value may not be a value that is present in the underlying table. For aggregate functions such as MIN and MAX, they are present, but for functions COUNT and SUM, they are not. This is the reason that this project uses auxiliary operations. 


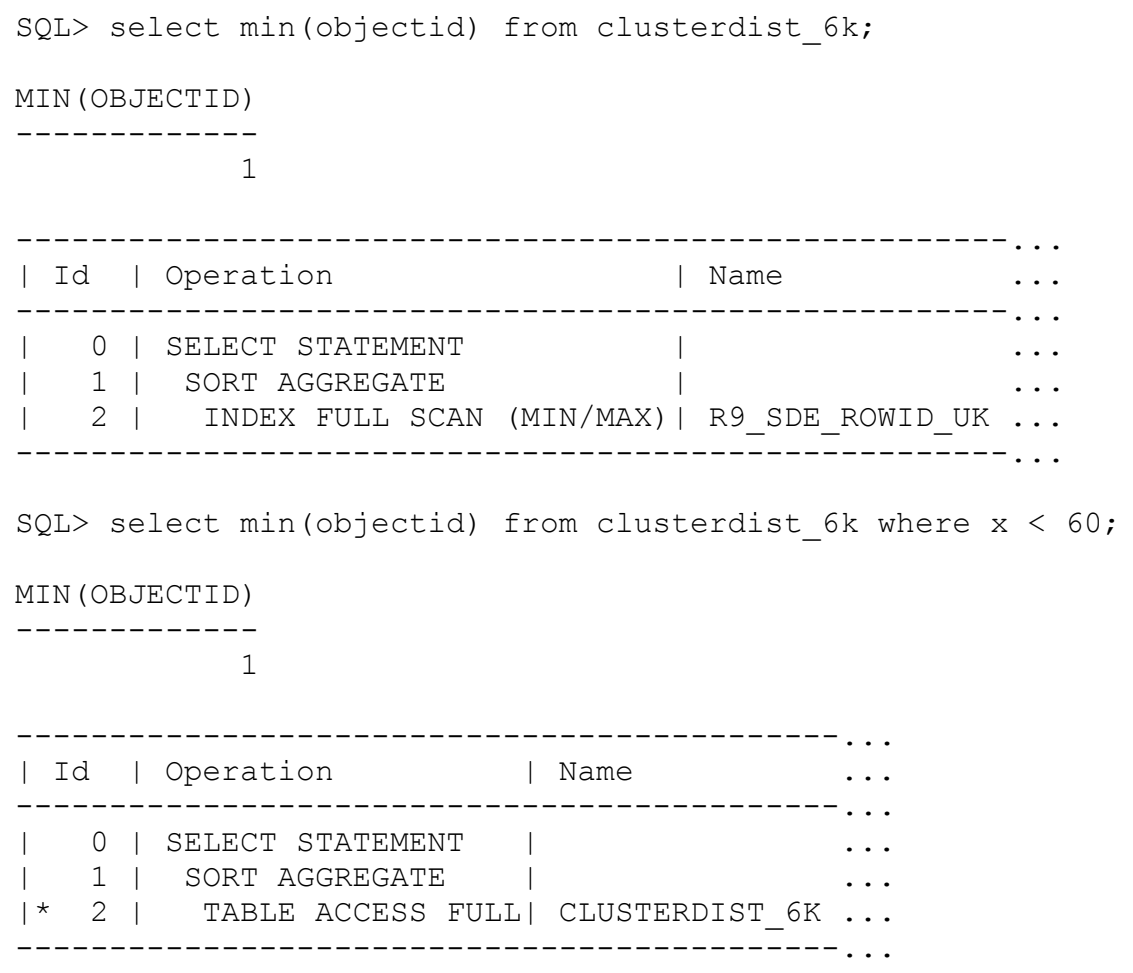

\section{Code Listing 5-1: These two statements illustrate the difference between getting the result from the index (first statement) and evaluating the table (second statement).}

The other limitation is much more serious. As complete filter information is not provided to the index, the index cannot omit any rows. For example, if a SQL statement were to query a company's PURCHASE_ORDER table for the maximum ORDER_VALUE where the STATE_NAME = 'California', an index on the ORDER_VALUE column may be used to find the maximum value. That value is then evaluated by the STATE_NAME = 'California' filter. If it happens to be in true, the correct answer is returned. If instead the STATE_NAME = 'New York', then nothing (NULL) is returned. It is for this reason rows cannot be omitted from the result set. With the built-in Oracle aggregate functions, this is not an issue as INDEX FULL SCANs are only performed when there are no other filter predicates. Similarly, user-defined aggregate operators cannot encounter this issue as they are never index aware, always being evaluated last. Auxiliary operators can access indexed metadata, improving their performance, but that index metadata has not had additional filters applied to it. If the full WHERE clause of the SQL statement were to be provided to the operator, it could choose not to include that index metadata. Unfortunately, as that filter information is not provided, the operator can either not take advantage of the index metadata, or risk returning incorrect or null results. For the operators implemented by this project, this limitation does not cause null information to be returned, but rather incorrect information. The values returned are always for the entire population, no matter what where clause is provided to the SQL statement. 


\subsubsection{Index Type}

The index type is defined by the ST_STAT_INDEX type and created by the ST_STAT_INDEX_TYPE.sql (type specification) and ST_STAT_INDEX.sql (type body) files. The Oracle Data Cartridge defines the member functions for this type. The major functions are listed in Table 5-1 and full descriptions of signatures and arguments are listed in Appendix A.

Table 5-1. The public functions and their uses in the ST_STAT_INDEX type.

\begin{tabular}{l|l}
\hline Name & Function \\
\hline ODCIIndexCreate & Creates the index \\
ODCIIndexStart & Called to start the index \\
ODCIIndexFetch & Called to fetch row id from the index \\
ODCIIndexClose & Called to close the index when finished \\
ODCIIndexInsert & Modifies the index after an insert \\
ODCIIndexUpdate & Modifies the index after a update \\
ODCIIndexDelete, & Modifies the index after a delete \\
ODCIIndexDrop & Drop the index \\
\hline
\end{tabular}

Each of these methods is fully described in the code comments, Appendix A and the Oracle Data Cartridge documentation. The majority of the work is done in the ODCIIndexCreate, ODCIIndexStart, and ODCIIndexFetch methods.

In the ODCIIndexCreate method, the type of index is identified, and the index table is created. The creation is done with a single Create Table As Select (CTAS) statement that aggregates the point data set according to the grid size of the spatial index. Without a spatial index, this will fail.

The ODCIIndexStart method invokes the spatial index ODCIIndexStart method and accesses the data structures it creates, specifically the SPX_UTIL.GRID_CELL_DEM_T table object that contains the relational information about the query shape and grid cells. The method then iterates this object once to find the interior of the boundary ratio if needed, and again to build the needed data structures that are required by the auxiliary operators.

There are limitations on the type of data that can be stored within a TYPE object in Oracle. Therefore, the majority of the aggregation work is done in the ST_STAT_UTIL package. This package contains a number of variables that hold information between the time when ODCIIndexStart is called and the first row is fetched. During that time, these session variables must remain intact. If this system were to support multiple concurrent SQL statements, this structure would have to change.

The ODCIIndexFetch method will perform one or two actions depending on the operating mode. If the system is estimating the results, this method simply returns the results from the spatial index ODCIIndexFetch. If the system is solving the result, then the function iterates all results from the spatial index ODCIIndexFetch, testing each against a list of boundary ROWIDs from the ODCIIndexStart method. For those that 
match, the row values are added to the aggregated totals that are provided to the auxiliary operators.

The index type is what took the majority of the development time. The reason for this is Oracle lacks a pass by reference. Every function or procedure call is a pass by value. This may not seem significant, but in the original implementation, a private function was used to update the interior or boundary counts. This in turn copied the entire data structure for every index cell that was evaluated, resulting in a worst-case run time that would scale exponentially. There is very little documentation on the lack of pass by reference. In fact, to diagnose this issue, components were removed from the solution until the issue was narrowed to the internal function call. The problem was then reproduced with a very simple Oracle package. To work around this problem all grid cell iteration is done in a single procedure that is contained in the same Oracle package that contains the aggregate grid memory structures.

\subsubsection{Mean Center}

The mean center operator, implemented by the package MEAN_CENTER, combines the sum of $x$, sum of $y$ and count variables from the index context to return the mean value in each direction. The ST_MEAN_CENTER_TYPE.sql (package header) and ST_MEAN_CENTER (package body) files generate the package.

Table 5-2. The auxiliary operators implemented using functions from the ST_MEAN_CENTER package.

\begin{tabular}{|c|c|}
\hline Name & Function \\
\hline ST_MEAN_CENTER & Returns the mean center as a geometry \\
\hline ST_MEAN_CENTER_X & Returns the $\mathrm{X}$ portion of the mean center \\
\hline ST_MEAN_CENTER_Y & Returns the Y portion of the mean center \\
\hline ST_MEAN_CENTER_PLUS_X_ERROR & $\begin{array}{l}\text { Returns the positive } X \text { portion of the } \\
\text { estimated mean center error }\end{array}$ \\
\hline ST_MEAN_CENTER_PLUS_Y_ERROR & $\begin{array}{l}\text { Returns the positive Y portion of the } \\
\text { estimated mean center error }\end{array}$ \\
\hline ST_MEAN_CENTER_MINUS_X_ERROR & $\begin{array}{l}\text { Returns the negative } X \text { portion of the } \\
\text { estimated mean center error }\end{array}$ \\
\hline ST_MEAN_CENTER_MINUS_Y_ERROR & $\begin{array}{l}\text { Returns the negative Y portion of the } \\
\text { estimated mean center error }\end{array}$ \\
\hline
\end{tabular}

Each of the operators listed in Table 5-2 are created using methods in the ST_MEAN_CENTER package. These call a central method named GET_OBJECT which calculates the index context variables. This way the result is calculated on the first call to the function, and the same value is returned on each subsequent call. The GET_OBJECT method has two sections controlled by different Boolean flags. The first calculates the main result for the operator, whether it is an exact or estimated value. It is controlled by the isSet variable. The second part calculates the error associated with the 
main result. This is controlled by the isEstimateSet set value, and will only be calculated if one of the estimate functions are called.

Finally, there is the cleanup method that is called from the index cleanup method. This method resets all the flags and values when the execution ends.

\subsubsection{Median Center}

The median center auxiliary operator is created using the ST_MEDIAN_CENTER_TYPE.sql (package header) and ST_MEDIAN_CENTER.sql (package body) files. Similar to the mean center implementation, these files create a package named MEDIAN_CENTER.

Table 5-3. The auxiliary operators implemented using functions from the ST_MEDIAN_CENTER package.

\begin{tabular}{|c|c|}
\hline Name & Function \\
\hline ST_MEDIAN_CENTER & $\begin{array}{l}\text { Returns the median center as a } \\
\text { geometry }\end{array}$ \\
\hline ST_MEDIAN_CENTER_X & $\begin{array}{l}\text { Returns the } \mathrm{X} \text { portion of the median } \\
\text { center }\end{array}$ \\
\hline ST_MEDIAN_CENTER_Y & $\begin{array}{l}\text { Returns the Y portion of the median } \\
\text { center }\end{array}$ \\
\hline ST_MEDIAN_CENTER_PLUS_X_ERROR & $\begin{array}{l}\text { Returns the positive } \mathrm{X} \text { portion of the } \\
\text { estimated median center error }\end{array}$ \\
\hline ST_MEDIAN_CENTER_PLUS_Y_ERROR & $\begin{array}{l}\text { Returns the positive } \mathrm{Y} \text { portion of the } \\
\text { estimated median center error }\end{array}$ \\
\hline ST_MEDIAN_CENTER_MINUS_X_ERROR & $\begin{array}{l}\text { Returns the negative X portion of the } \\
\text { estimated median center error }\end{array}$ \\
\hline ST_MEDIAN_CENTER_MINUS_Y_ERROR & $\begin{array}{l}\text { Returns the negative Y portion of the } \\
\text { estimated median center error }\end{array}$ \\
\hline
\end{tabular}

Again, similar to the mean center implementation, each of the operators listed in Table 53 are created using functions in the ST_MEADIAN_CENTER package, which calls a GET_OBJECT method. This is where the value is generated and stored in the index context. The GET_OBJECT method in this case uses the point count, ST_STAT_UTIL.COLUMN_COUNTS and ST_STAT_UTIL.ROW_COUNTS table objects to determine where the median point is. This is done by iterating the count tables to find the column or row that contains the needed point, and then ordering the spatial index table rows in the given row or column to access the median point. The error value is generated by the same procedure, except the ST_STAT_UTIL.B_COLUMN_COUNTS and ST_STAT_UTIL.B_ROW_COUNTS are also used.

During the implementation of this method, two programming errors required significant amount of effort to diagnose. The first was a typo in the SQL statement to find the $y$ value of the median point. Due to a copy/paste error, this SQL statement was 
actually finding the correct point, but returning the incorrect dimension. The second was a logical problem, where the implementation was accounting for the boundary counts when finding the median point count, but not when attempting to find the row that contained it. This works to return the exact result, as there are no boundary cases. When estimating, this error would ignore the boundary counts leading to results that were slightly off.

\subsubsection{Standard Distance}

Standard distance is implemented in two files: ST_STD_DIST_TYPE.sql (package header) and ST_STD_DIST.sql (package body). The same pattern is used, where functions call a GET_OBJECT function that actually calculates all the required information.

Table 5-4. The auxiliary operators implemented using functions from the ST_STD_DIST package.

\begin{tabular}{l|l}
\hline Name & Function \\
\hline ST_STD_DIST & $\begin{array}{l}\text { Returns the standard distance of the population } \\
\text { Returns a geometry that is a circle with radius equal to } \\
\text { the standard distance, centered at the mean center of the } \\
\text { population } \\
\text { The positive error of the standard distance estimate } \\
\text { The negative error of the standard distance estimate }\end{array}$ \\
ERROR_PLUS &
\end{tabular}

This package calls the mean center package to determine the mean center of the population, the sum of $x$-squared value and the sum of $y$-squared value. To generate the error value of the result, each boundary cell is evaluated for proximity to the mean center. If the closest corner of the cell is further than the standard distance, the cell is counted towards the positive error. If the furthest corner of the cell is closer than the standard distance, it is counted towards the negative error.

\subsubsection{Directional Distribution}

The implementation of directional distribution was unsuccessful. It is technically possible to implement this solution, but the limitations of computer systems-specifically floating-point numbers - cause the current approach to be impossible. This is caused by the loss of precision in the float values that are used to store the aggregated totals. For mean center and standard distance, any possible error is only visible at the $10^{\text {th }}$ or $15^{\text {th }}$ decimal place. This error is far less than the majority of data collection devices, and as such, can be safely ignored. In the case of standard direction, the equation requires that the difference between two terms have a square root generated from it. These terms are the result of the sum of $x$ squared by $y$ squared summation values (i.e., $\operatorname{Sum}\left(x^{*} x^{*} y^{*} y\right)$ ) and can grow to values that exceed the floating points ability to accurately store them. The sensitivity of this equation results in this function being unstable on all but the 
smallest datasets. There may be a method to calculate the needed values without loss of precision, but ultimately this was found to be outside this project's scope.

\section{Table 5-1: The auxiliary operators implemented using functions from the} ST_DIR_DIST package.

\begin{tabular}{l|l}
\hline Name & Function \\
\hline ST_DIR_DIST_GEOMETRY & $\begin{array}{l}\text { Returns the geometry that represents the } \\
\text { directional distribution } \\
\text { The length of the directional distributions major } \\
\text { axis } \\
\text { The length of the directional distributions minor } \\
\text { axis }\end{array}$ \\
ST_DIR_DISTRIBUTION_MAJOR_DISTRIBUTION_MINOR \\
The angle of rotation of the major axis
\end{tabular}

With the complexity of the equations needed to generate the results for directional distribution, the major component of this implementation was simply correctly entering the mathematical equation. This took significantly more time than was expected. This, in addition to the diagnosis of the underlying cause of the floating-point errors outlined above, resulted in this operator being the most time consuming to implement.

\subsection{Index-Only Functions}

While the auxiliary operator implementation performs reasonably well, it is still constrained by the Oracle Data Cartridge implementation. It still needs to return all the expected rows, and as such a large amount of work that is done by the underlying spatial index. This is unlike the original Oracle behavior that this project is attempting to replicate. If an aggregate function, such as MIN or MAX, is used against a column with an index, the result can wholly be returned by the index and the base table does not need to be accessed. Unfortunately, this behavior is unavailable in the current Oracle Data Cartridge. This behavior can be partially simulated by a function that accesses the index directly. This function would perform all the actions needed to determine the spatial relationship of the grid cells and the query shape, but not actually build the list of resulting rows. This is a significant optimization, but has a number of major limitations.

A function that is designed to return only the statistical result is technically simpler than the auxiliary operators are, but this is both its strength and weakness. The lack of restrictions allows the function to access the index and return the value types that are required. It no longer has to return a subset of specific rows (like the index) or accept specific arguments (like the operators). However, this lack of restrictions also prevents different function classes from sharing information, meaning each function call must create its own aggregated information from the spatial index. It also means that this function is called by selecting the result of a function, rather than a select statement from a table. 


\subsection{Testing}

The data used to test these operators were generated using scripts. Each script was designed to produce a $6,000,60,000,600,000$, and $6,000,000$ point datasets in each of a random, uniform, and clustered distribution. This yields twelve distinct datasets. The extent of all the datasets range from 140 to 40 degrees west and 10 to 70 degrees north. These scripts are listed in Appendix A. A description of the datasets including counts, database blocks and row length is included in the Appendix $\mathrm{C}$.

The uniform script generates a square grid of cells at a given interval. The interval is varied to generate close to the required number of points. The random script generates the exact number of points, using an Oracle random function to generate the $\mathrm{X}$ and $\mathrm{Y}$ coordinates. The cluster script iterates the Countries, U.S.A. states, and Canadian provinces that intersect the dataset extent. The source is an administrative boundaries feature class. The script then randomly inserts points in the extent of the given polygon feature. The number of insertions per feature is controlled by the population of that feature, and normalized to result in the needed number of records. This results in a feature class that roughly approximates the population distribution of North America.

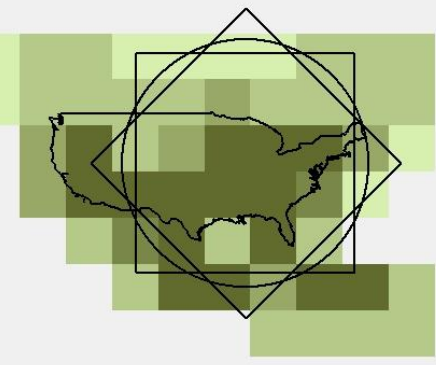

Row count per cell $(\min / \max ): 1 / 759$ Total (cells / rows): 54 / 6,018

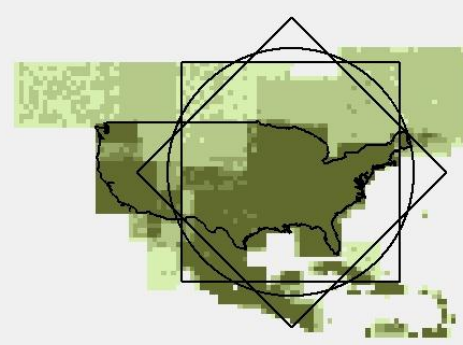

Row count per cell $(\min / \max ): 1 / 13714$

Total (cells / rows): 3,473 / 601,921

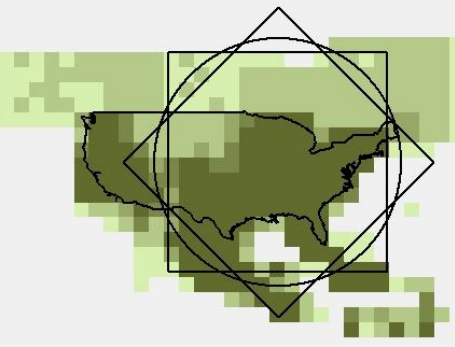

Row count per cell (min / $\max ): 1 / 3163$ Total (cells / rows): 394 / 60,191

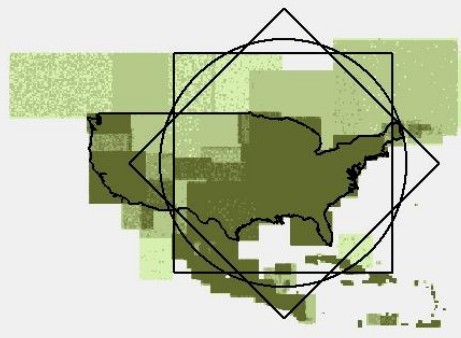

Row count per cell (min / max): 1 / 63,073 Total (cells / rows): 33,267 / 6,019,198

Figure 5-1. Detailed information about the clustered datasets, with the query shapes overlaid 
There are four filter shapes used to test the data. They are a square, circle, diamond and the continental U.S.A. These four shapes exist in a separate feature class and are used as query shapes whenever testing is done. Figures 5.1 through 5.3 illustrate the distribution of the different dataset and their relationship with the query shapes.

The test is performed in three steps. The first executes the Mean Center or Standard Distance Geoprocessing Tool through a command line program. This program uses shared methods with the unit-testing framework to evaluate the implemented operators. The execution of this geoprocessing tool on one of the point datasets, using a filter based on one of the query shapes, is logged and the results recorded. When a geoprocessing tool is not present or fails to yield results, the value is created using a SQL statement that employs Oracle aggregate operators. In the case of median center, this is the only method that is possible.

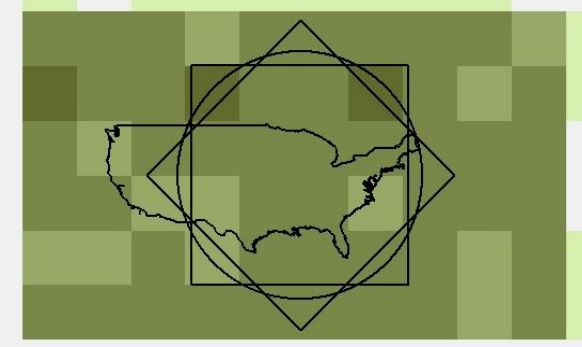

Row count per cell $(\min / \max ): 1 / 125$

Total (cells / rows): 72/ 6,000

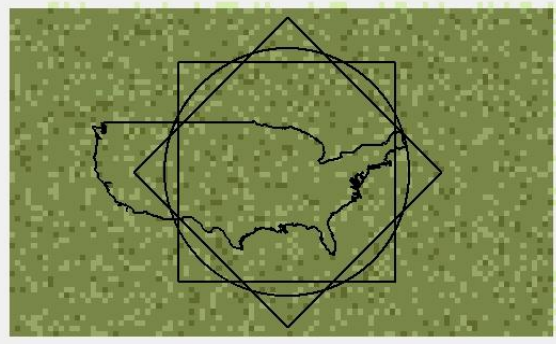

Row count per cell (min / $\max ): 1 / 134$

Total (cells / rows): 6,045/600,000

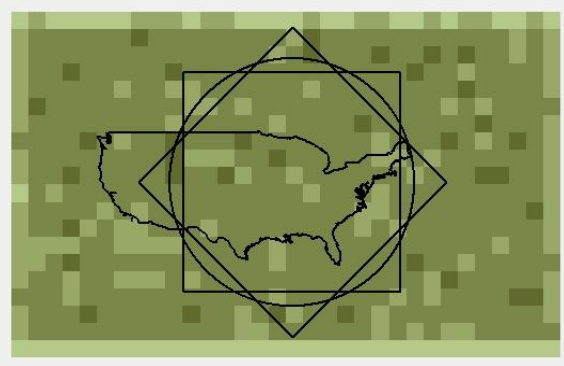

Row count per cell ( $\min / \max ): 24$ / 640 Total (cells / rows): 394 / 60,000

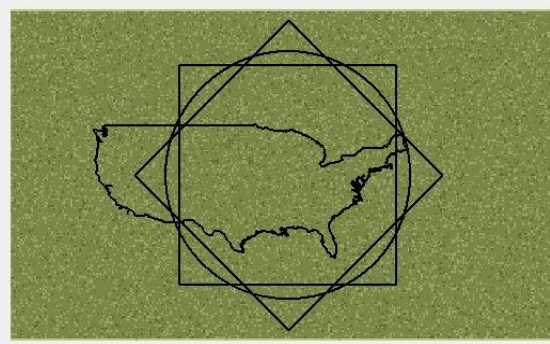

Row count per cell ( $\min / \max ): 11 / 145$ Total (cells / rows): 60,547/ 6,000,000

Figure 5-2. Detailed information about the randomly distributed datasets, with the query shapes overlaid 


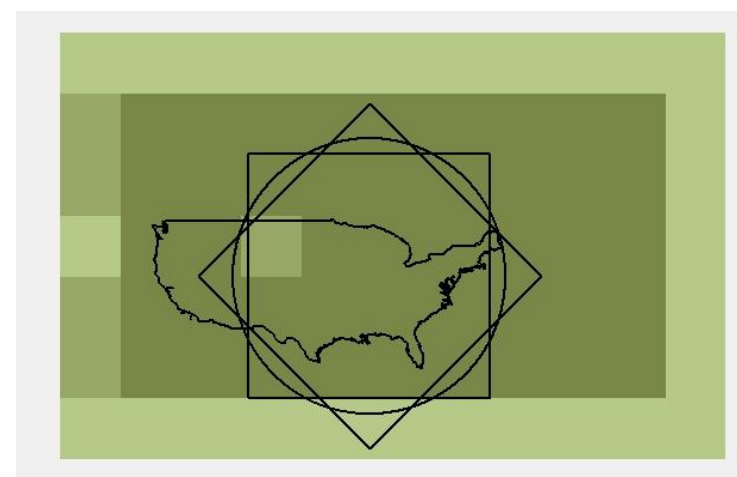

Row count per cell (min / max): 20 / 100

Total (cells / rows): 77 / 6,000

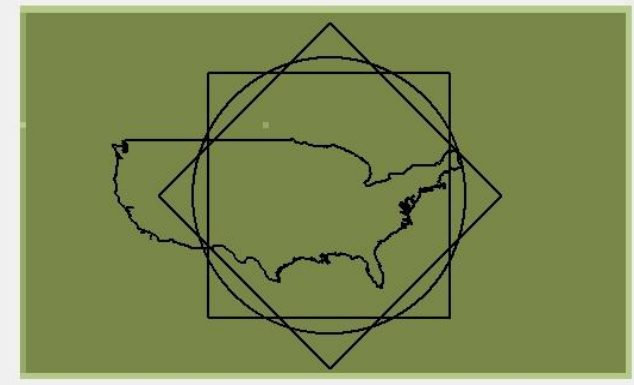

Row count per cell (min / max): 20 / 100

Total (cells / rows): 6,161 / 600,000

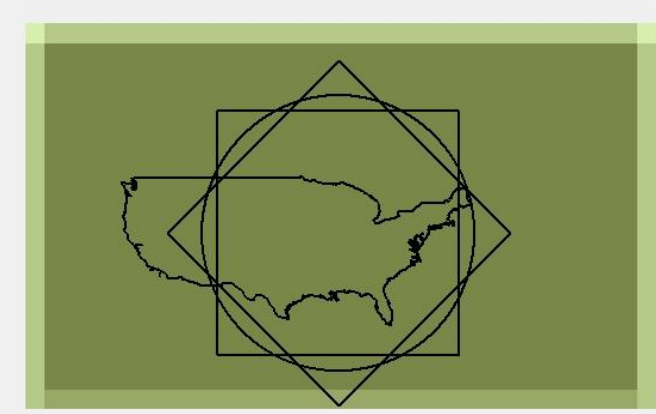

Row count per cell $(\min / \mathrm{max}): 6 / 100$

Total (cells / rows): 660 / 60,230

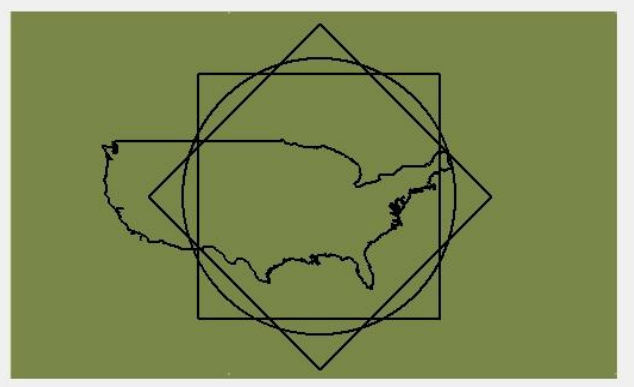

Row count per cell ( $\min / \max ): 45 / 100$

Total (cells / rows): 60,230 / 6,003,374

Figure 5-3. Detailed information about the uniformly distributed datasets, with the query shapes overlaid

Using either the geoprocessing or SQL results as a baseline, the operator is executed and timed. The results are compared to the baseline value and the test either passes, when the value returned is within the tolerance of the test, or fails, when the value is not returned within 0.0005 units.

\subsection{Results}

Results were collected using a C\# unit-testing application that ran different combinations of operators, solve methods, distributions, and population sizes. This application first established a connection to the Oracle database, cleared the database shared pool and buffer caches, and then executed the same SQL statement twice. There were three different sets of functions run against three different distributions of four different population sizes. These combinations for all but the variation in dataset size are outlined in Table 5-6. 
Table 5-6. A listing of all operators and functions that had their performance data recorded

\begin{tabular}{llll}
\hline Function Name & Implementation & Results & Is Estimate? \\
\hline Mean & & & \\
Oracle (avg) & Oracle & X and Y values & $\mathrm{N}$ \\
User defined & This project & ST_Geometry & $\mathrm{N}$ \\
Auxiliary - Solve & This project & ST_Geometry & $\mathrm{N}$ \\
Auxiliary - Estimate & This project & ST_Geometry & $\mathrm{Y}$ \\
Auxiliary - Error & This project & ST_Geometry and error & $\mathrm{Y}$ \\
Median & & values & \\
Oracle (median) & Oracle & ST_Geometry & $\mathrm{N}$ \\
Auxiliary - Solve & This project & ST_Geometry & $\mathrm{N}$ \\
Auxiliary - Estimate & This project & ST_Geometry & $\mathrm{Y}$ \\
Auxiliary - Error & This project & ST_Geometry and error & $\mathrm{Y}$ \\
Standard Distance & & values & \\
Oracle (sum and avg) & Oracle & & $\mathrm{N}$ \\
Auxiliary - Solve & This project & Number & $\mathrm{N}$ \\
Auxiliary - Estimate & This project & Number & $\mathrm{Y}$ \\
Auxiliary - Error & This project & Number and error values & $\mathrm{Y}$ \\
\hline
\end{tabular}

For each of these operators or functions, the test application executes a SQL statement twice to exercise the function or operator in a cold (no data cached) or hot (with data cached) state. Before the execution, the Oracle package DBMS_MONITOR is activated to record the system statistics for the current session. This enables the test application to capture 26 different performance indicators. The test application then adds an additional 10 values specific to accuracy of the operators. Table 5-7 lists the indicators that are used to evaluate this solution. A detailed listing of all indicators is available in Appendix C.

The detailed analyses of these results are contained in the following chapter. At a high level, the implemented auxiliary operators outperformed Oracle aggregate operators when run against a cold dataset with optimal spatial index grid sizes. Once the data was contained within the database cache, operators performed marginally better. Using an index-only function significantly outperformed both the auxiliary operators and Oracle aggregate functions. Unfortunately, the limitations of the index-only functions do not make them a viable solution. 
Table 5-7. The listing of data metrics used when analyzing the performance of this solution

\begin{tabular}{|c|c|c|}
\hline Indicator Name & Units & Description \\
\hline \multicolumn{3}{|l|}{ Oracle Recorded Values } \\
\hline Session logical reads & Disk blocks & $\begin{array}{l}\text { The number of disk blocks Oracle has to } \\
\text { fetch from disk or memory }\end{array}$ \\
\hline Physical reads & Disk blocks & $\begin{array}{l}\text { The number of disk blocks Oracle has to } \\
\text { fetch from the physical disk, including both } \\
\text { data and temporary tablespaces }\end{array}$ \\
\hline \multicolumn{3}{|c|}{ Test Application Recorded Values } \\
\hline X Value & Geometry Units & The $X$ value of the result \\
\hline Y Value & Geometry Units & The Y value of the result \\
\hline $\mathrm{X}$ Variation & Geometry Units & $\begin{array}{l}\text { The difference between the } X \text { value and the } \\
\text { expected } X \text { value }\end{array}$ \\
\hline Y Variation & Geometry Units & $\begin{array}{l}\text { The difference between the } \mathrm{Y} \text { value and the } \\
\text { expected Y value }\end{array}$ \\
\hline Net stopwatch & Seconds & The time elapsed in the test application \\
\hline SQL Error & Boolean & $\begin{array}{l}1 \text { if there was an error in the test } \\
\text { application or SQL statement; otherwise } 0\end{array}$ \\
\hline Pos X Expected Error & Geometry Units & $\begin{array}{l}\text { The amount of error in the positive } \mathrm{X} \\
\text { direction }\end{array}$ \\
\hline Pos Y Expected Error & Geometry Units & $\begin{array}{l}\text { The amount of error in the positive } \mathrm{Y} \\
\text { direction }\end{array}$ \\
\hline Neg X Expected Error & Geometry Units & $\begin{array}{l}\text { The amount of error in the negative } \mathrm{X} \\
\text { direction }\end{array}$ \\
\hline Neg Y Expected Error & Geometry Units & $\begin{array}{l}\text { The amount of error in the negative } \mathrm{Y} \\
\text { direction }\end{array}$ \\
\hline
\end{tabular}




\section{Chapter 6 - Results and Analysis}

This chapter discusses the results and draws conclusions based on the performance experienced while testing the previously described system. This system demonstrates shortcomings in the Oracle Data Cartridge, but the methods it proposes are sound; the amount of data processing is reduced and the reuse of spatially cached data increases efficiency. This in essence, is how a spatial index works. This project extended that spatial indexing structure, facilitating the storage information that can be combined for multiple purposes. Testing demonstrates a $50 \%$ to $90 \%$ decrease in processing time caused by an up to a $98 \%$ reduction in disk activity.

\subsection{Auxiliary Operators}

The auxiliary operators all behaved in similar and expected ways. This is characterized by performance gains over conventional aggregate functions for the first query of datasets containing roughly 600,000 records or more, with the amount of gain being proportional to the size of the dataset. Subsequent queries performed roughly the same as the Oracle aggregate functions. The accuracy of the auxiliary estimate operators' results were within the maximum estimated values. The amount of variation between the estimate and the actual result was dependent on the grid size of the underlying index, which is dependent on the density of the point features and the distribution of the point dataset.

\subsubsection{Dataset Distributions}

One of the goals of this project was to decrease the amount of data read from disk when calculating certain statistical metrics. In an Oracle database, all rows are stored within database blocks. A single block may contain zero or many database rows. The block is the smallest unit that the Oracle database processes will read from disk and cache into memory. When the database requires a row (or set of rows) to satisfy a query, the block is requested using a consistent get. If that block has already been read and is still in the database buffer cache, the block is retrieved from the cache, and no disk read is performed. If that block is not present in the database buffer cache, a physical read is performed, and the block is cached. Blocks are aged out of the buffer cache using a least used algorithm. This means that the goal of the project was not only to reduce the number of rows needed, but also the number of blocks needed from disk. This is an important point, as the organization of rows and blocks can have a dramatic effect on the performance of a query.

The organization of rows and blocks was the reason why the randomly distributed data performs worse than clustered and uniform datasets in the following discussion. The uniform dataset creation scripts create the data in rows and columns, with records created consecutively across each row. This effectively sorted the data on disk. The random dataset creation script performed random inserts across the same geographic extent. This created a random distribution on disk. The different load orders affect performance by changing the number and order of blocks that the query has to retrieve. With sorted data, 
rows that exist in the same grid cell are more likely to exist in the same database block. This means that one disk read will likely contain a set of rows that will be needed in the near future. With random data, these rows are more likely to be in different blocks, increasing the number of reads from disk. The clustered distribution falls somewhere between the uniform and random distribution. When created, the script will insert points randomly in a variety of geographic extents. This creates a distribution of rows that are partially sorted with rows randomly distributed in clustered groups. For more a detailed explanation of the data used, please refer to Section 5.3.

\subsubsection{Underlying Spatial Index}

In each test against a dataset containing 6 million points, there are 60-70 thousand block reads performed by the underlying spatial index. These statements are counted as recursive SQL statements. Table 6-1 lists the components of the overall cost for the recursive and non-recursive statements for a mean center query, using the ST_Mean auxiliary function and the AVG Oracle aggregate function, run against 6 million randomly distributed points.

Table 6-1. The cost of the recursive and non-recursive portions of Auxiliary and Aggregate mean center operations

\begin{tabular}{lccccc}
\hline & \multicolumn{2}{c}{ Auxiliary } & & \multicolumn{2}{c}{ Aggregate } \\
\cline { 2 - 3 } \cline { 5 - 6 } & Non-Recursive & Recursive & & Non-Recursive & Recursive \\
\hline Elapsed & 7.53 & 75.62 & & 903.26 & 79.92 \\
Disk Blocks & 240 & 66834 & & 129206 & 64054 \\
Cache Blocks & 13021 & 56166 & & 1606338 & 64982 \\
File Read & $0.08(212)$ & $68.68(40563)$ & & $882.90(129021)$ & $72.40(40645)$ \\
Exproc & $1.03(3)$ & $0(0)$ & & $1.04(3)$ & $0(0)$ \\
\hline
\end{tabular}

Note. Elapsed time is in seconds. File Read and Exproc values represent the number of seconds waited for that resource and the number of times that resource was waited on.

Table 6-1 illustrates the performance gains through the use of the auxiliary operators. Specifically, the number of disk reads performed by the auxiliary operators is less than $0.2 \%$ that of the aggregate functions, resulting in the time waiting for a file read dropping from almost 15 minutes to less than a tenth of a second.

\subsubsection{Query Shapes}

The Query shape appears to have less effect on the performance and the estimate accuracy than the consistency of the shape's boundary. With a regular shape (square, diamond or circle) the number of boundary cells is minimized due to the straight, or consistent, edges. With irregular shapes (USA), the number of boundary cells can increase because the edge meanders, intersecting additional cells. 
Table 6-2. Number of interior and boundary grid cells for 6 million point datasets, and the average execution time using Estimate or Solve approaches

\begin{tabular}{|c|c|c|c|c|c|}
\hline \multirow[t]{2}{*}{ Shape } & \multicolumn{2}{|c|}{ Average Execution Time } & \multirow[t]{2}{*}{ Boundary } & \multirow[t]{2}{*}{ Interior } & \multirow[t]{2}{*}{ Total } \\
\hline & Estimate & Solve & & & \\
\hline Square & 15.7 & 17.3 & 604 & 15625 & 16129 \\
\hline Diamond & 26.3 & 28.2 & 713 & 15665 & 16378 \\
\hline Circle & 22.3 & 24.4 & 568 & 15699 & 16267 \\
\hline USA & 67.0 & 73.7 & 1869 & 6861 & 8730 \\
\hline
\end{tabular}

The complexity of the USA query shape's edge results in queries that take longer to complete than the other queries. Both in the operators and functions implemented by this project and those it depends on in the underlying spatial type.

\subsection{Mean Center}

The mean center operator performs well across all the different dataset distributions. The largest gains are shown with the random distribution (Figure 6-3) and the smallest in the uniform distribution (Figure 6-1). The estimates produced by the auxiliary operators were consistent with the actual results provided by both auxiliary operators and aggregate functions. Expectedly, the accuracy of the estimates increased when the spatial index grid size decreased. The following three figures display the number of disk and cache blocks for each of the different solution methods and each of the different data distributions.

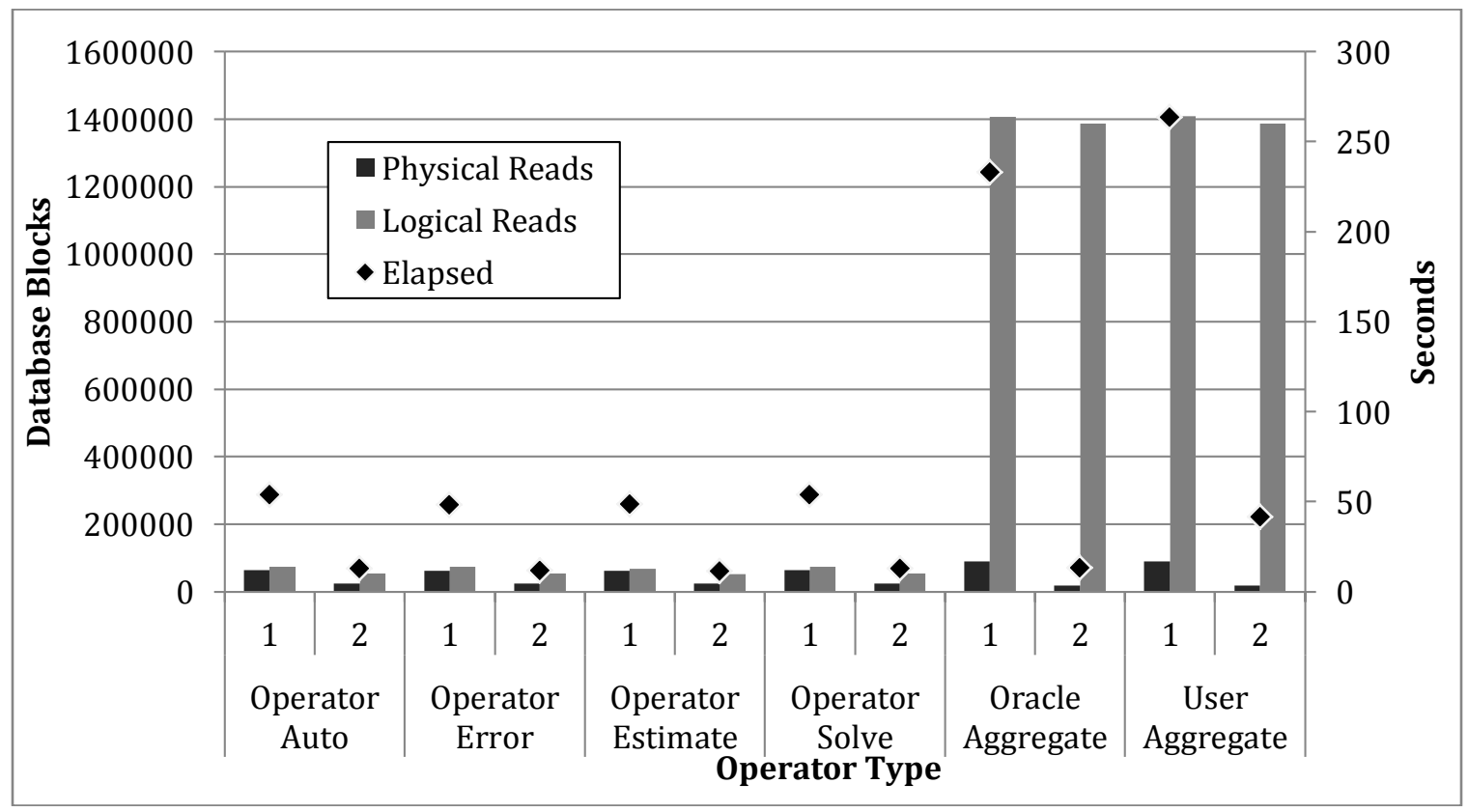

Figure 6-1. Performance data for the mean center calculation on a 6 million point uniformly distributed dataset using different evaluation methods. 


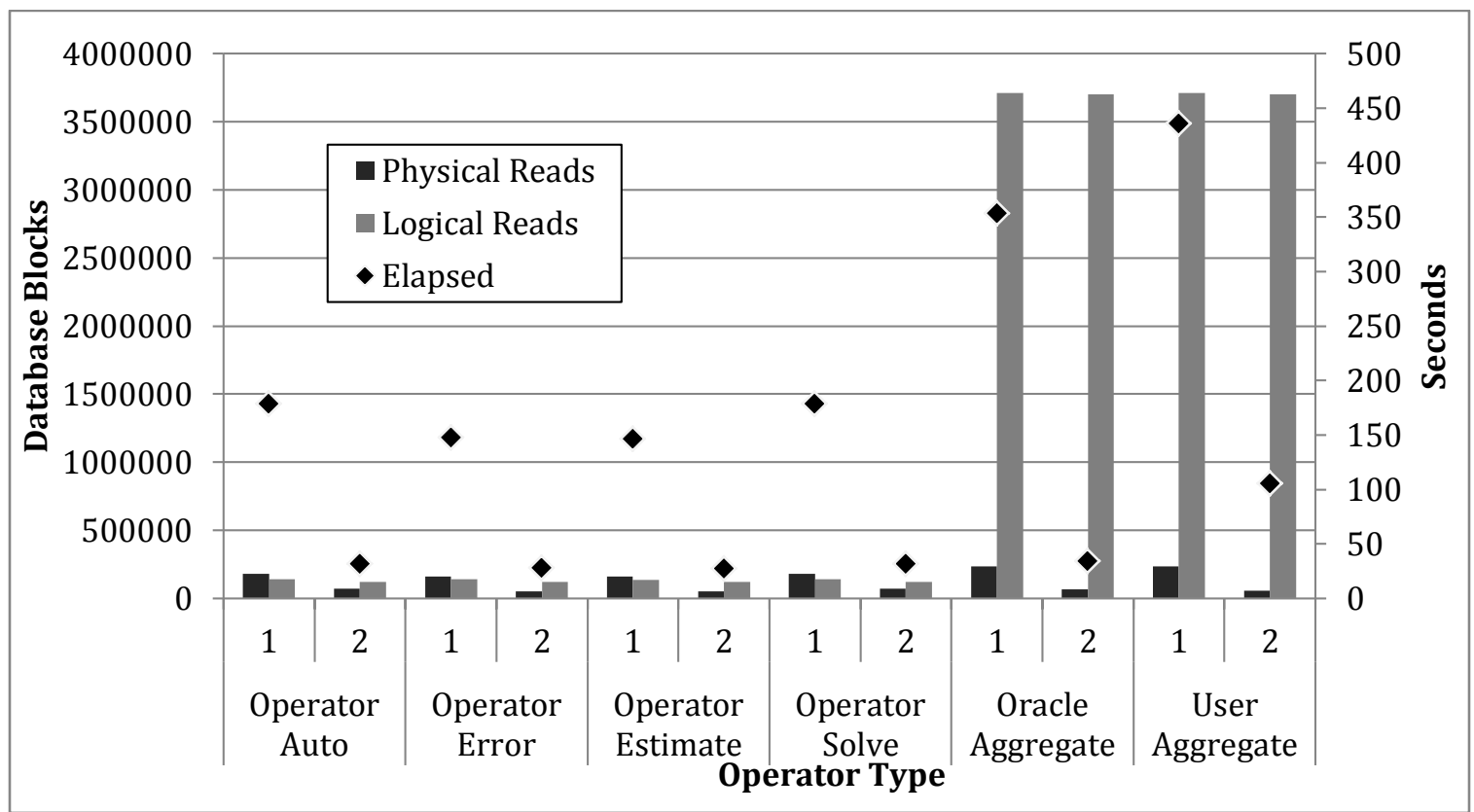

Figure 6-2. Performance data for the mean center calculation on a 6 million point clustered dataset using different evaluation methods.

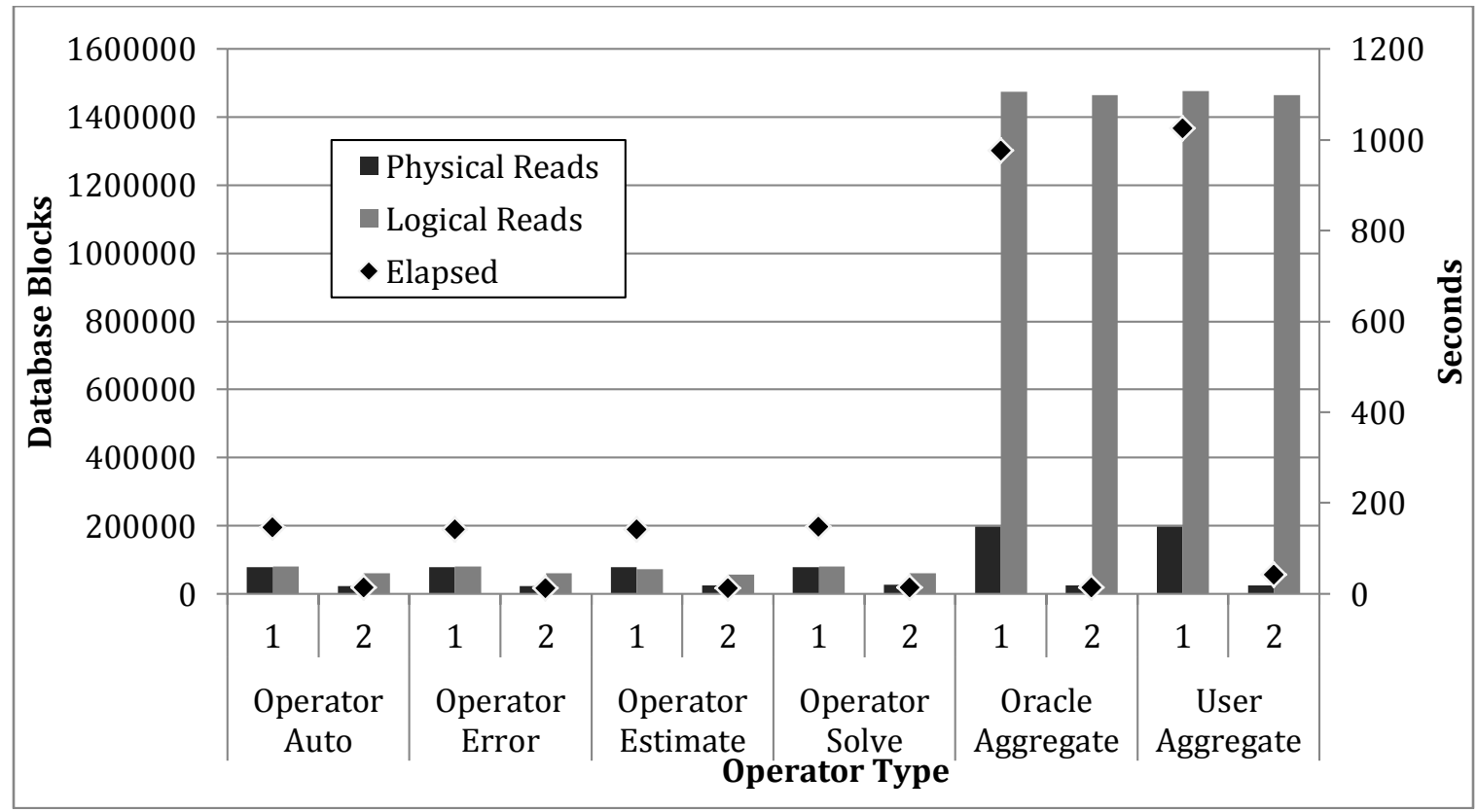

Figure 6-3. Performance data for the mean center calculation on a 6 million point randomly distributed dataset using different evaluation methods. 
The amount of variation between the actual result and the estimated result decreased as the index grid size decreased. The grid cell size is a function of the point population density. More points in a specific region results in a smaller grid sizes. As the estimate and error is based on the exclusion and inclusion of boundary grid cells, the smaller the grid cell, the smaller the effect on the overall estimate. The estimated error bounds and the variation from the actual result are presented in Figure 6-4.

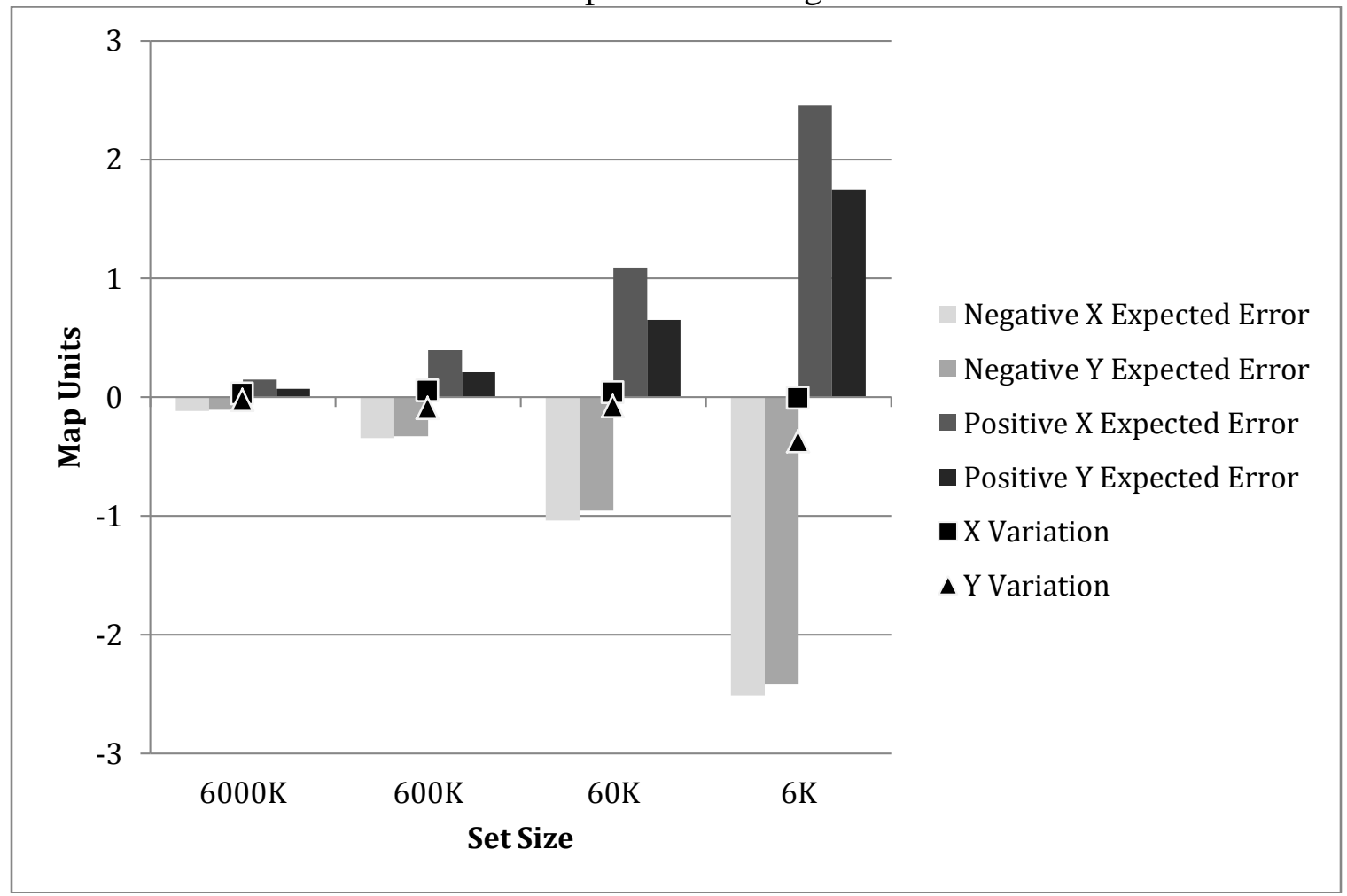

Figure 6-4. The variation between the estimated and actual mean center value and maxium error estimated by the operator, averaged across the different distirbutions and query shapes

Estimation functionality was originally included in this project due to expectations that finding the full solution to these metrics would be significantly more expensive. Through testing, it appears that this is not the case. The generation of a full solution does not appear to be significantly more expensive than allowing the index to determine the correct approach.

The behavior of the error result over different data distributions and shapes also behaves as expected. With uniform or random distributions, the estimate is close to the actual result. With the cluster distribution or irregular shapes, the estimates may vary significantly more from the result. This is caused by an uneven number of points contained by the boundary cells in a clustered distribution, or an irregular shape causing an increased number of boundary cells on either side of the query shape. Figure 6-5 illustrates this behavior. 

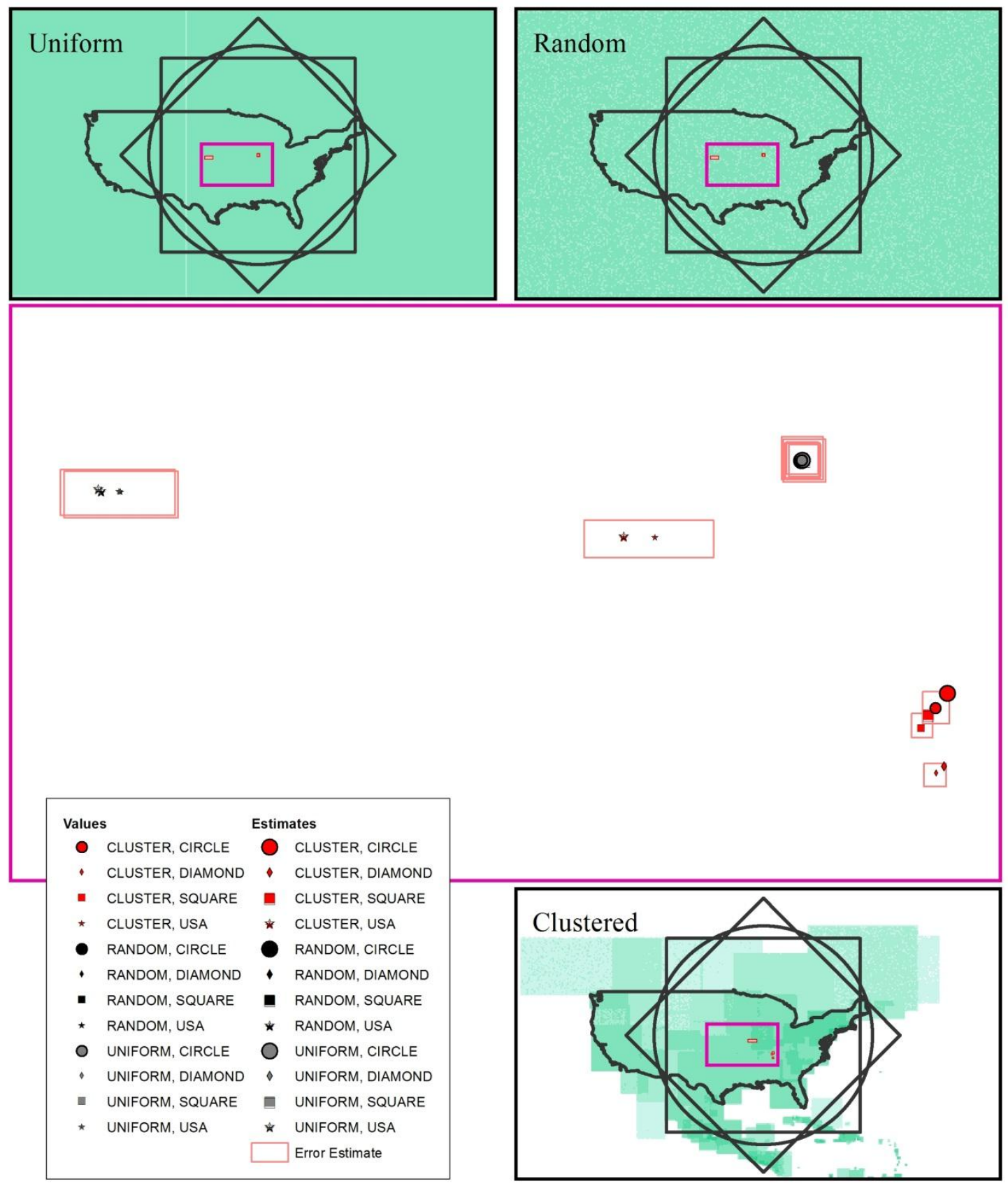

Figure 6-5. The ST_Mean value and error estimates for a 6 million point dataset for different query shapes and distribution combinations 


\subsection{Median Center}

Although the mean center operator aggregates values together and the median center operator sorts values, their performance is similar. Like the mean center operator, the median center operator outperforms the Oracle function on the first request, with the largest gains on random distribution (Figure 6-8), followed by the uniform (Figure 6-6) and cluster distributions (Figure 6-7). The estimates produced fall within the estimates provided, with the only major variation between the actual and estimated results generated against a clustered population.

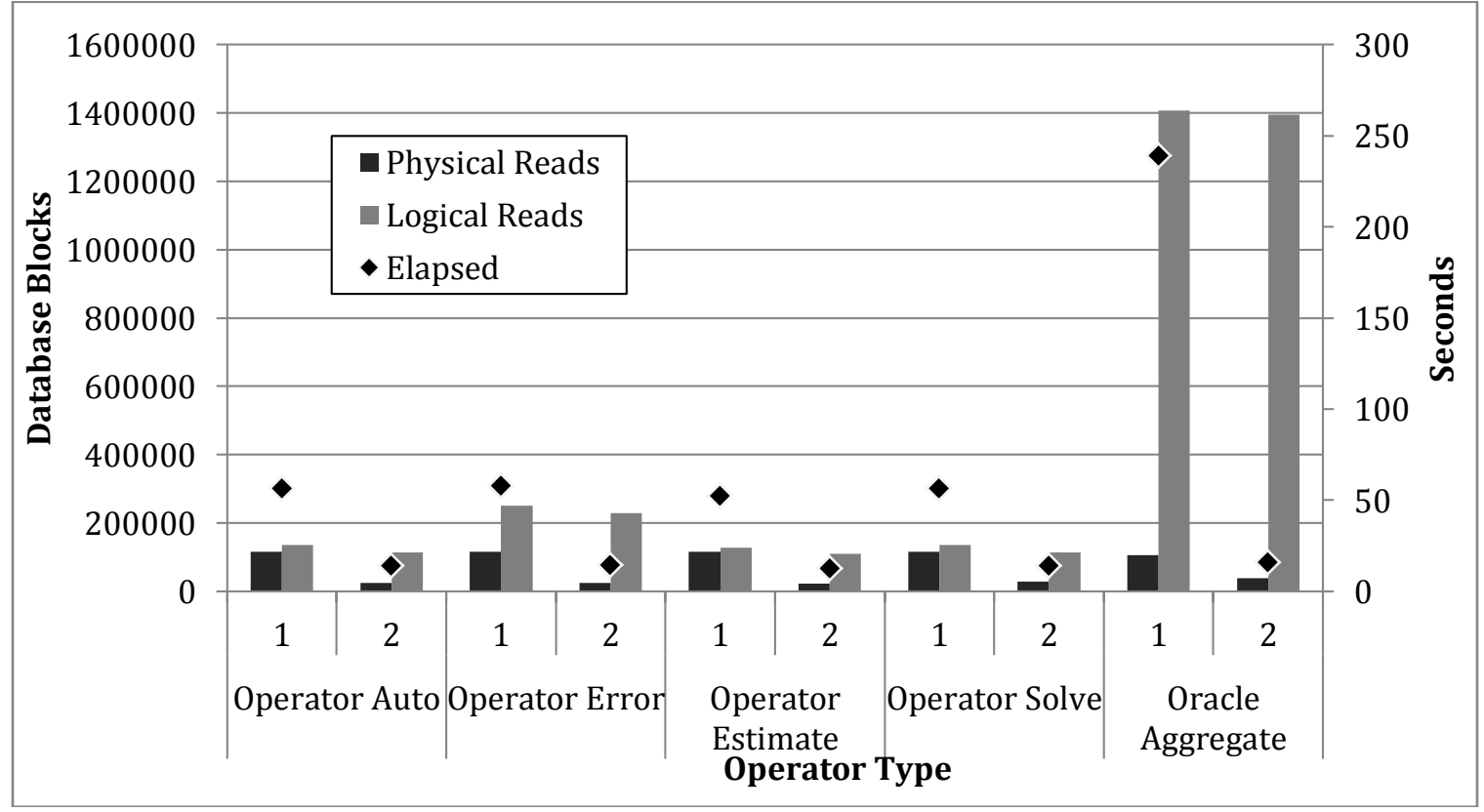

Figure 6-6. Performance data for the median center calculation on a 6 million point uniformly distributed dataset using different evaluation methods. 


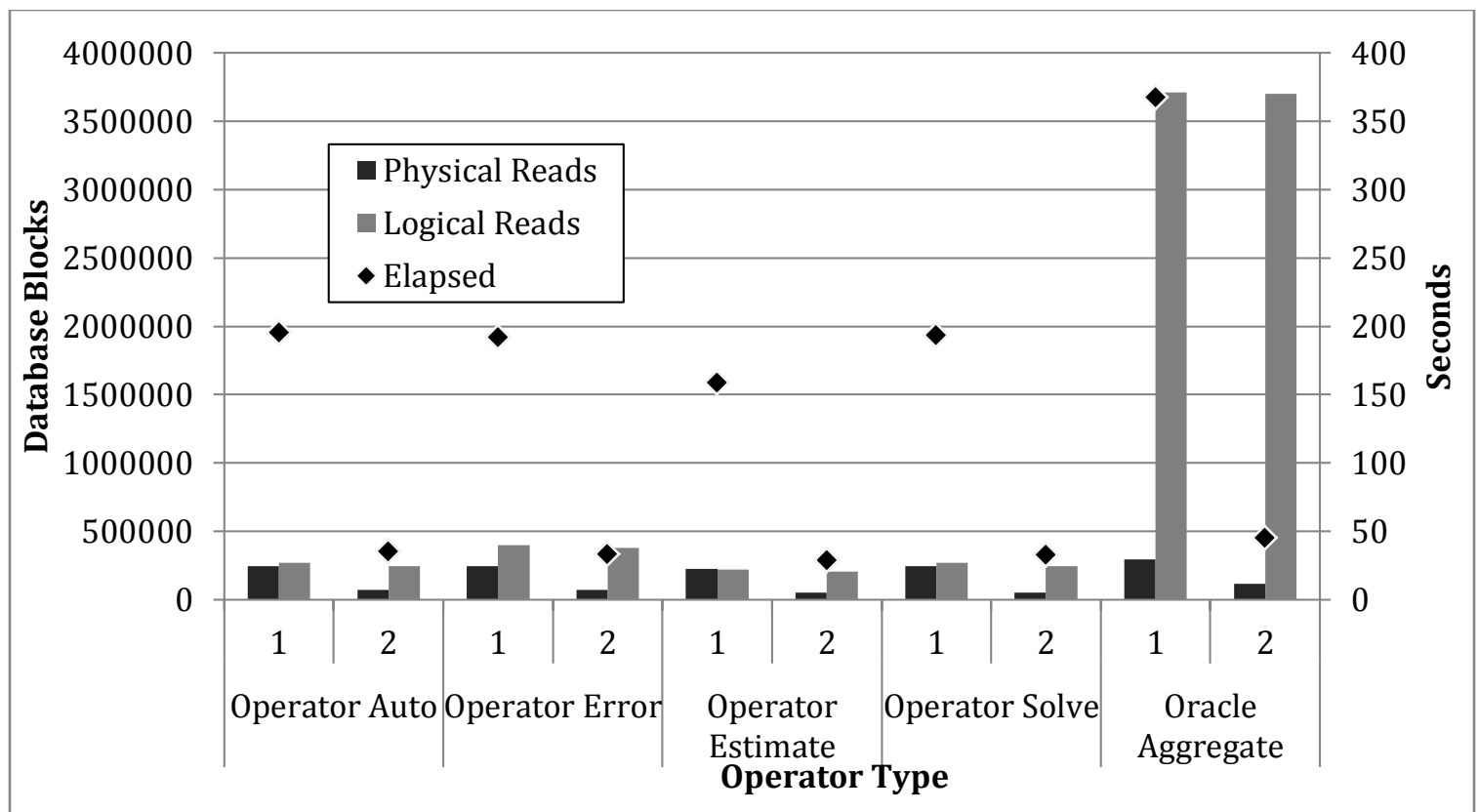

Figure 6-7. Performance data for the median center calculation on a 6 million clustered dataset using different evaluation methods.

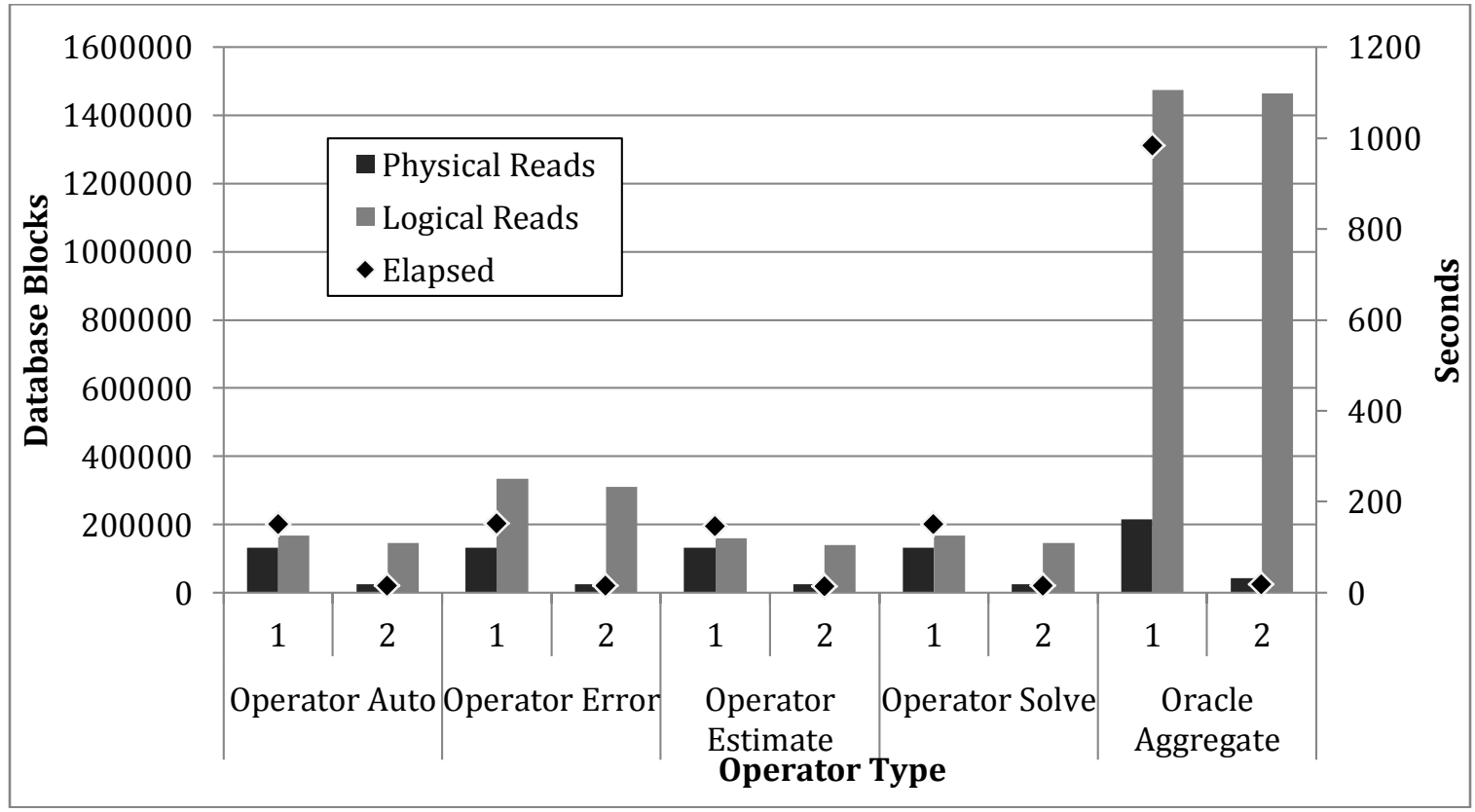

Figure 6-8. Performance data for the median center calculation on a 6 million point randomly distributed dataset using different evaluation methods. 


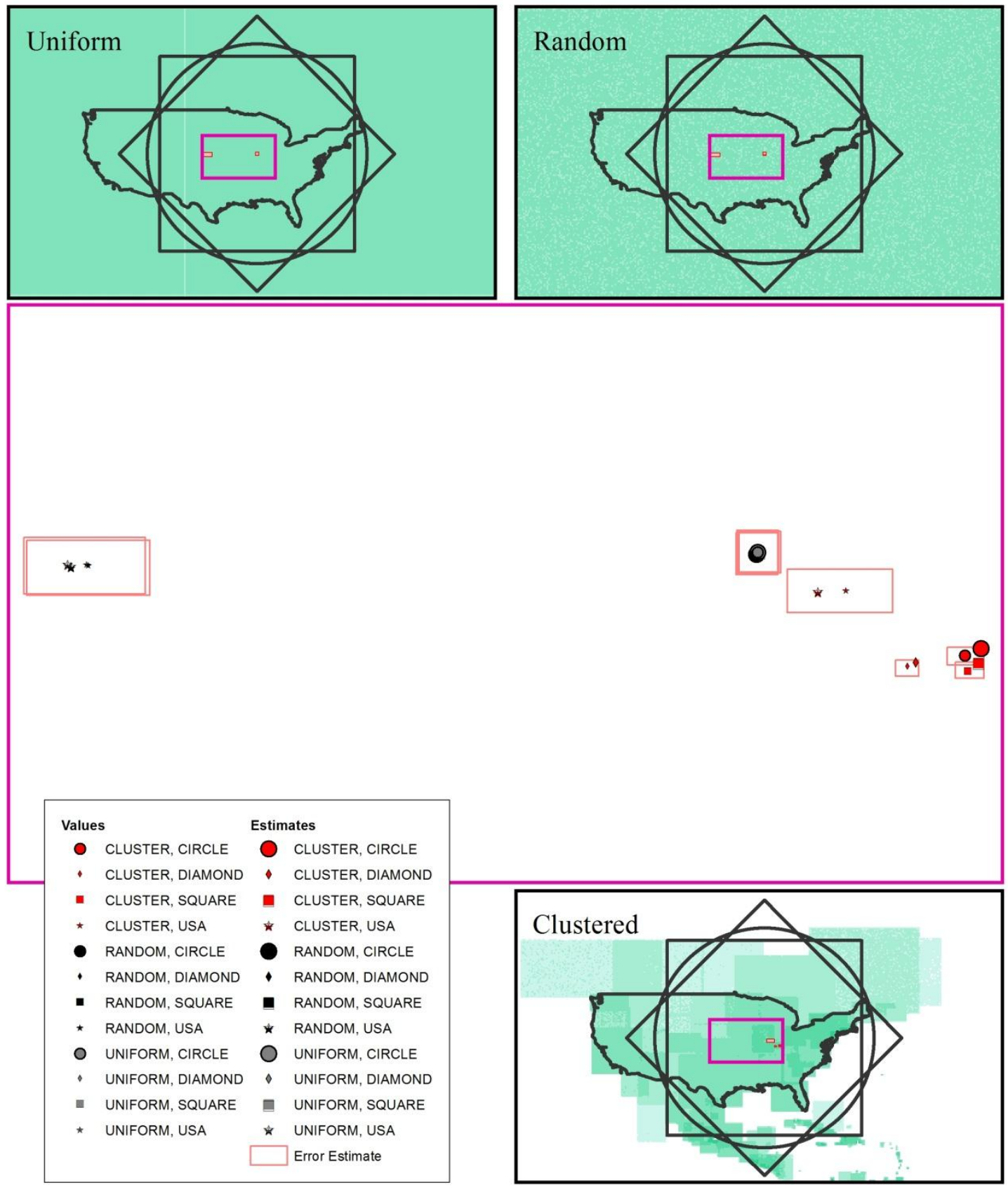

Figure 6-9. The ST_Median value and error estimates of a 6 million point dataset for different query shapes and distribution combinations. 


\subsection{Standard Distance}

Standard distance operates in an almost identical manner as mean center. As expected, the results from testing are almost identical. The auxiliary operators have significant performance gains over the Oracle function during the first execution. The effect of data distribution is the same as the mean center operator with the greatest performance gains observed on randomly distributed data (Figure -12), followed by uniform (Figure 6-10) and cluster (Figure 6-11) distributions.

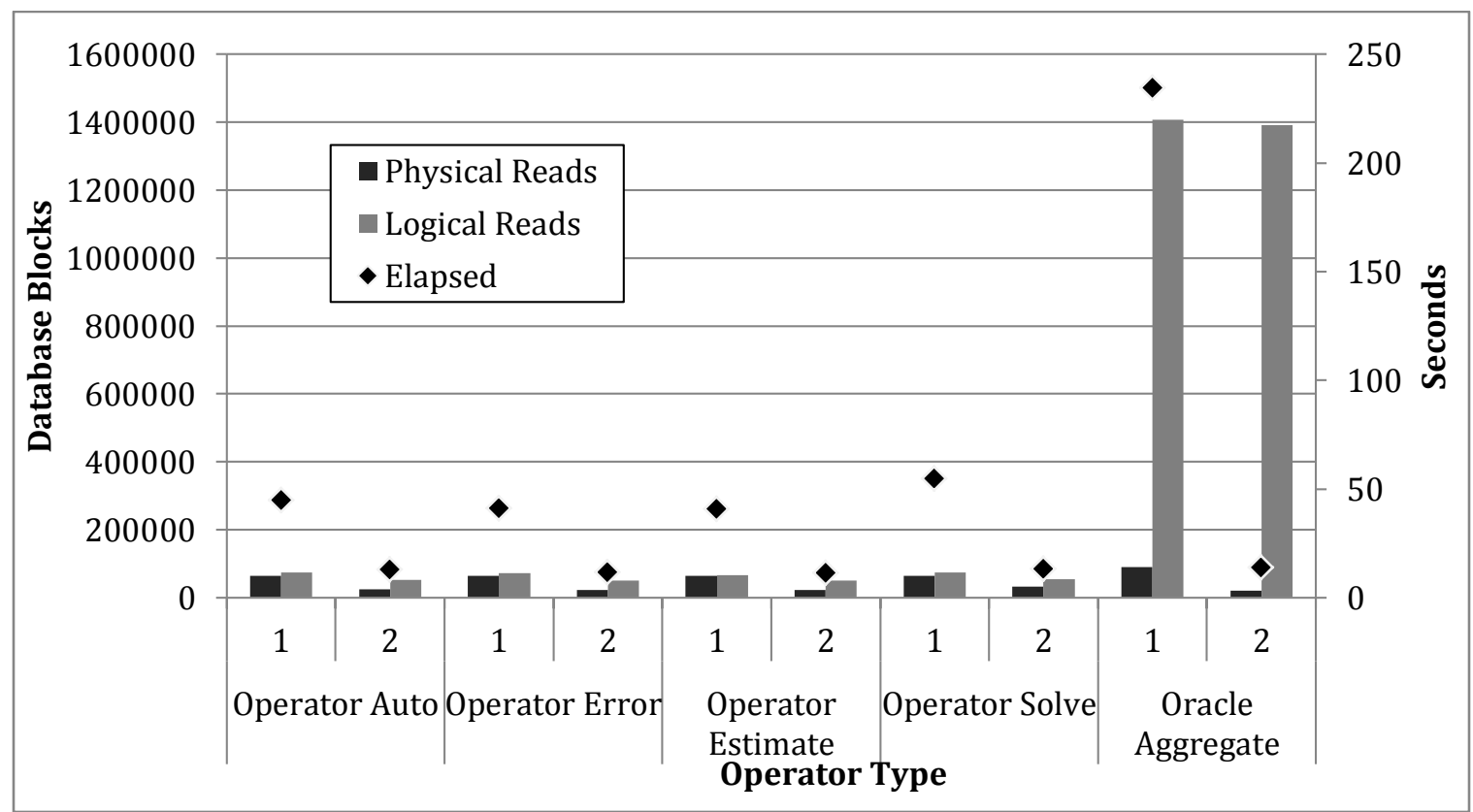

Figure 6-10. Performance data for the standard distance calculation on a 6 million point uniformly distributed dataset using different evaluation methods. 


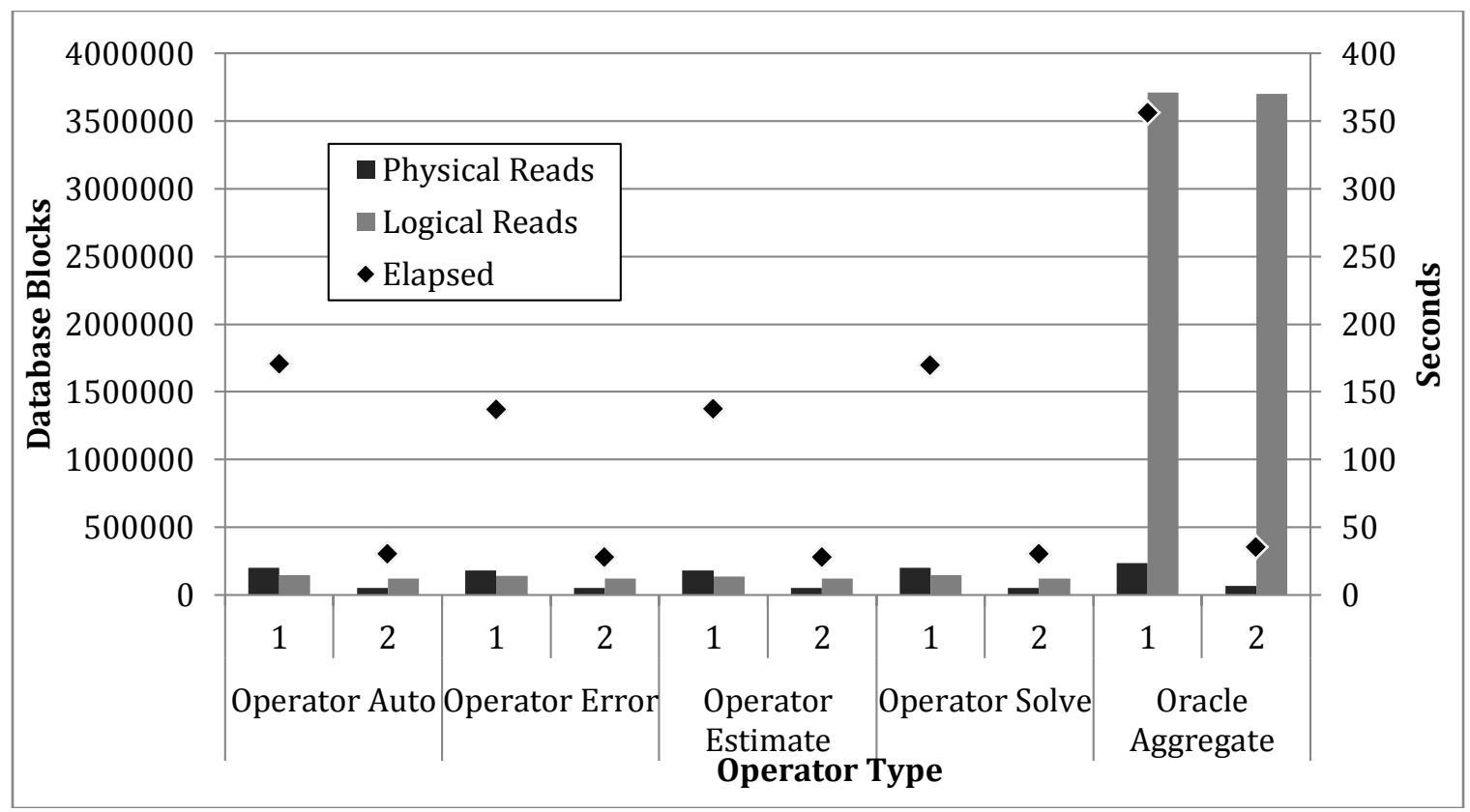

Figure 6-11. Performance data for the standard distance calculation on a 6 million point clustered dataset using different evaluation methods.

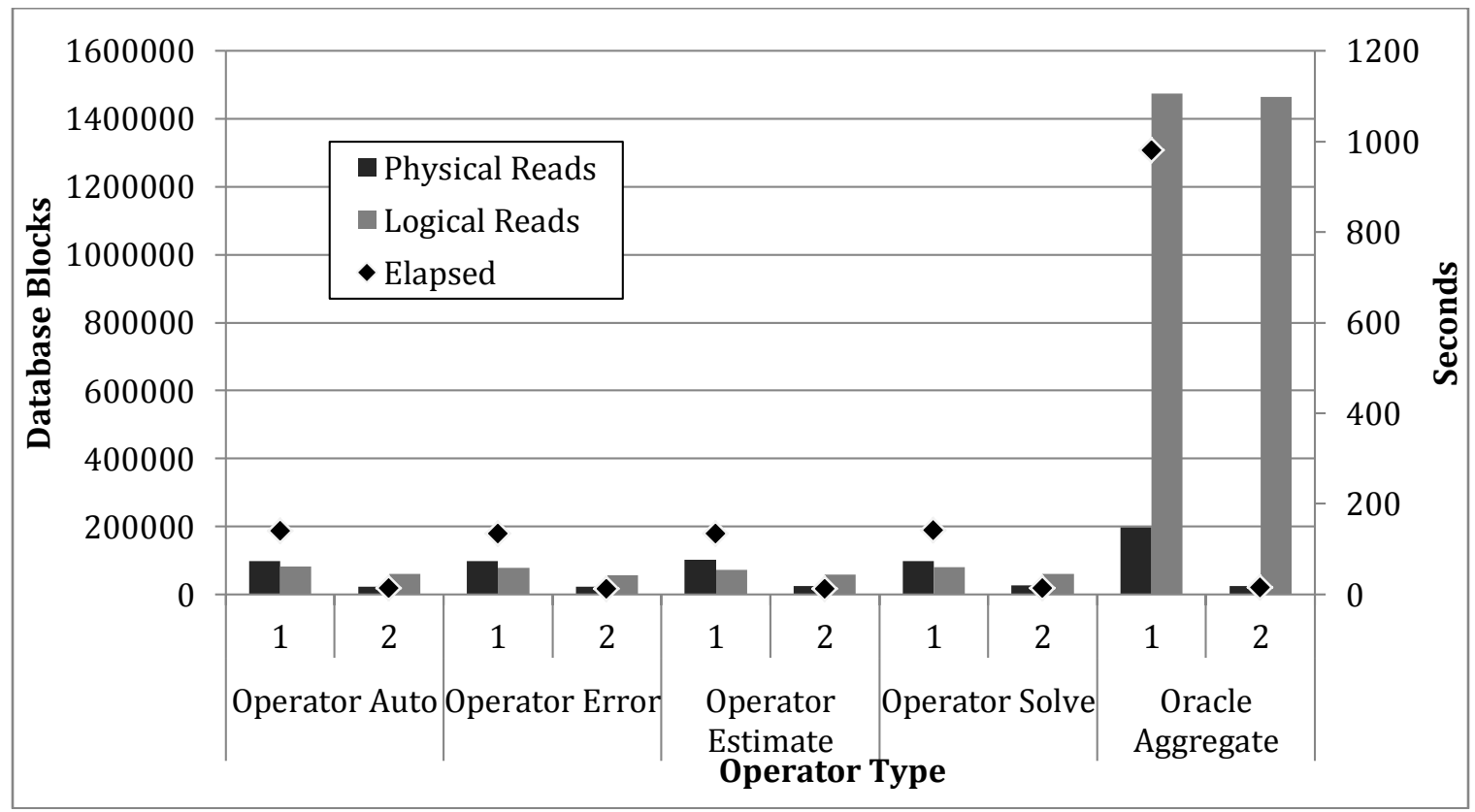

Figure 6-12. Performance data for the standard distance calculation on a 6 million point randomly distributed dataset using different evaluation methods. 
The estimates produced fall within the estimate error values produced. The amount of variation between the estimated and expected values is below 0.2 units for all except those cases using the USA query shape. This is caused by the increased number of boundary cells that this shape has. Unlike the other two operators that divide the boundary cells between two different directions (left/right or above/below the estimate), the standard distance operator uses cells closer and further to the mean center than the estimated value. Additionally, standard distance is actually a function of the mean center. Accounting for the variation in the estimate for the mean center and the variation in the standard distance estimate proved to be problematic.

The first attempts to display the estimate results involved buffering the estimated mean center value with the upper, estimated, and lower standard distance values. This was then compared with the result of the actual mean center buffered with the actual standard distance value. This resulted in a set of four circles, three concentric, and one that intersected the estimated value and lower bound circles. As the upper and lower estimate circles should actually bound the actual result, this was investigated. There was no error in the estimation logic, but rather a failure to account for the error introduced by the mean center estimate. For the upper bound, an attempt to correct for this was performed by buffering the polygon that represents the extent of the mean center error. For the lower bound, each of the corners of the mean center error extent was buffered by the minimum standard distance value. These polygons were then intersected to generate a shape that was thought to represent the lower bound of the possible standard distance estimate. This lower bound represents the area where all points are at most the minimum estimated value away from any point in the mean center's estimated extent. This process, although yielding a result in which the estimate circles did not intersect the standard circle, was misleading as it did not represent the area that should contain two thirds and a normally distributed dataset. The final approach was to generate four different sets of error estimates, recalculating the standard distance for each of the maximum bounds of the mean center estimate. In each case, only the cells that would contribute to the current estimate were considered. This yields the results presented in Table 6-3.

These values demonstrate that there is greater variation among different error estimates when the shapes do not have a consistent boundary, and when the datasets are not evenly distributed. The USA shape and clustered distribution demonstrate the largest variation between error estimates, while queries using other shapes against random or uniform distributions having almost no variation. These results are shown graphically for queries against a uniform and clustered distribution, using a square or the USA query shape in Figures 6-13 through 6-16. Additional graphical representations are available in Appendix E. 
Table 6-3. Value, estimate, and error bounds for standard distance operations on 6million point datasets. Neg., Pos., Pos. X, Neg. x, Pos. Y, Neg. Y are the variations in the error estimate for the negative overall, positive overall, lower left, lower right, upper left, and upper right error values respectively.

\begin{tabular}{l|cccccccc}
\hline Query & Value & Estimate & Neg. & Pos. & Pos. X & Neg. X & Pos. Y & Neg. Y \\
\hline $\begin{array}{c}\text { Circle } \\
\quad \text { Cluster }\end{array}$ & 12.565 & 12.392 & 0 & 0.327 & 0.027 & 0.302 & 0.031 & 0.297 \\
$\quad$ Random & 15.810 & 15.66 & 0 & 0.283 & 0.143 & 0.141 & 0.144 & 0.141 \\
$\quad$ Uniform & 15.818 & 15.679 & 0 & 0.285 & 0.144 & 0.142 & 0.144 & 0.143 \\
Diamond & & & & & & & & \\
$\quad$ Cluster & 13.360 & 13.23 & 0 & 0.276 & 0.027 & 0.245 & 0.125 & 0.155 \\
$\quad$ Random & 16.164 & 15.994 & 0 & 0.360 & 0.180 & 0.180 & 0.181 & 0.180 \\
$\quad$ Uniform & 16.153 & 15.979 & 0 & 0.365 & 0.181 & 0.185 & 0.185 & 0.181 \\
Square & & & & & & & & \\
$\quad$ Cluster & 12.950 & 12.819 & 0 & 0.234 & 0.048 & 0.189 & 0.024 & 0.211 \\
$\quad$ Random & 16.139 & 15.947 & 0 & 0.259 & 0.130 & 0.130 & 0.130 & 0.130 \\
$\quad$ Uniform & 16.260 & 16.189 & 0 & 0.258 & 0.129 & 0.130 & 0.130 & 0.129 \\
USA & & & & & & & & \\
$\quad$ Cluster & 15.089 & 14.834 & 0.108 & 0.547 & 0.169 & 0.372 & 0.365 & 0.197 \\
$\quad$ Random & 14.165 & 13.783 & 0.061 & 0.789 & 0.552 & 0.234 & 0.533 & 0.283 \\
$\quad$ Uniform & 14.167 & 13.778 & 0.060 & 0.804 & 0.564 & 0.238 & 0.536 & 0.298 \\
\hline
\end{tabular}




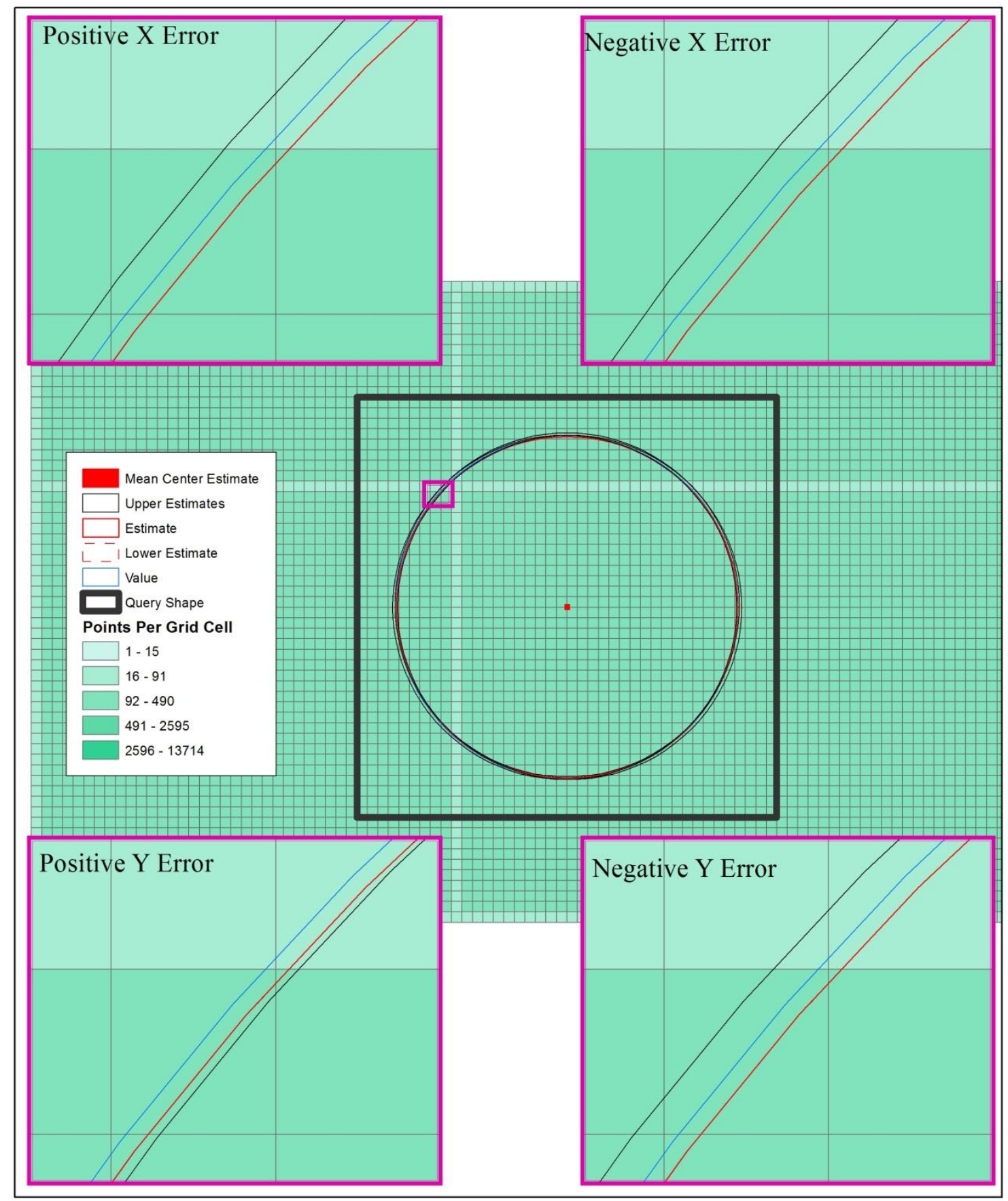

Figure 6-13. Error estimates of standard distance against a square query shape and a uniform distribution 

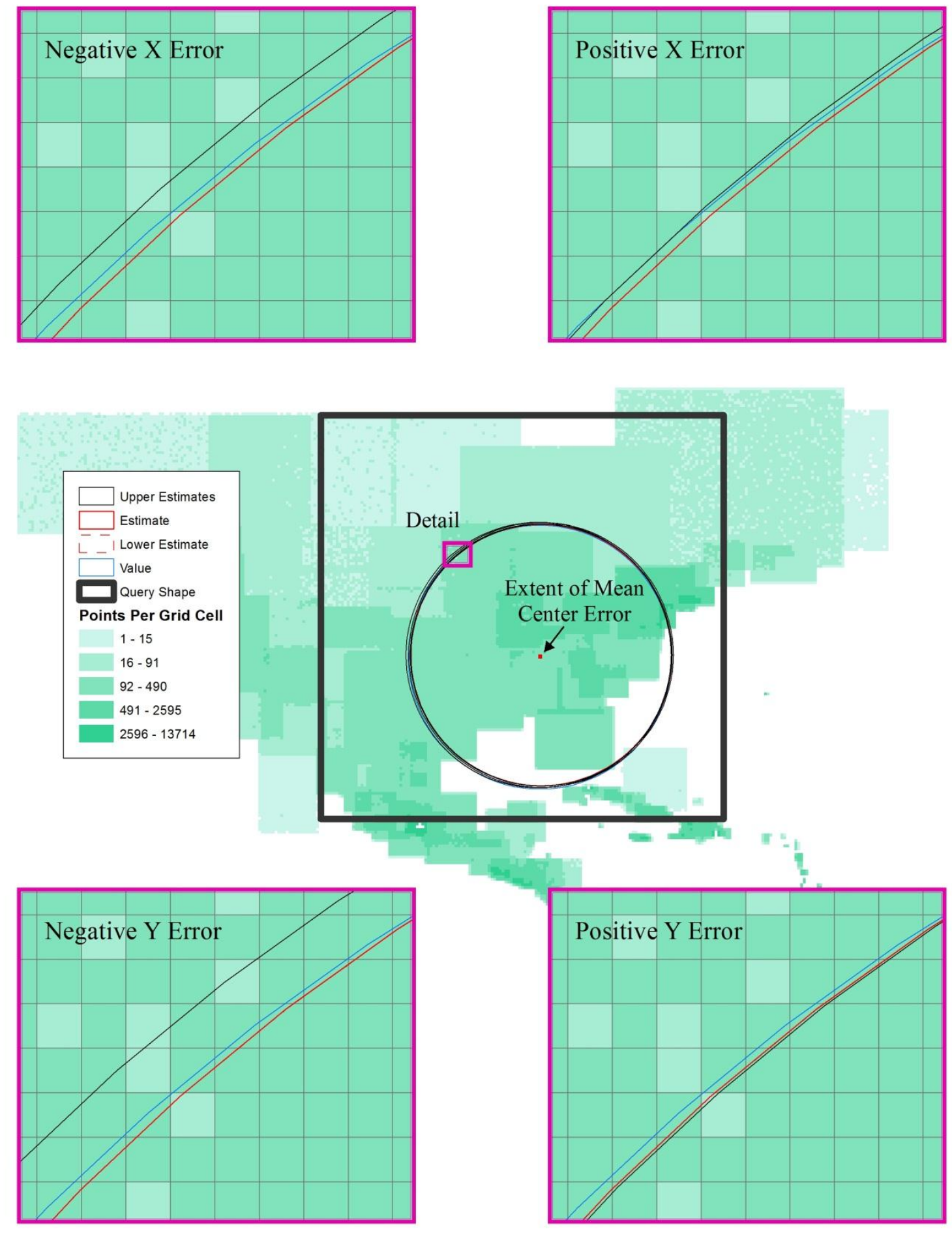

Figure 6-14. Error estimates of standard distance against a square query shape and a clustered distribution 


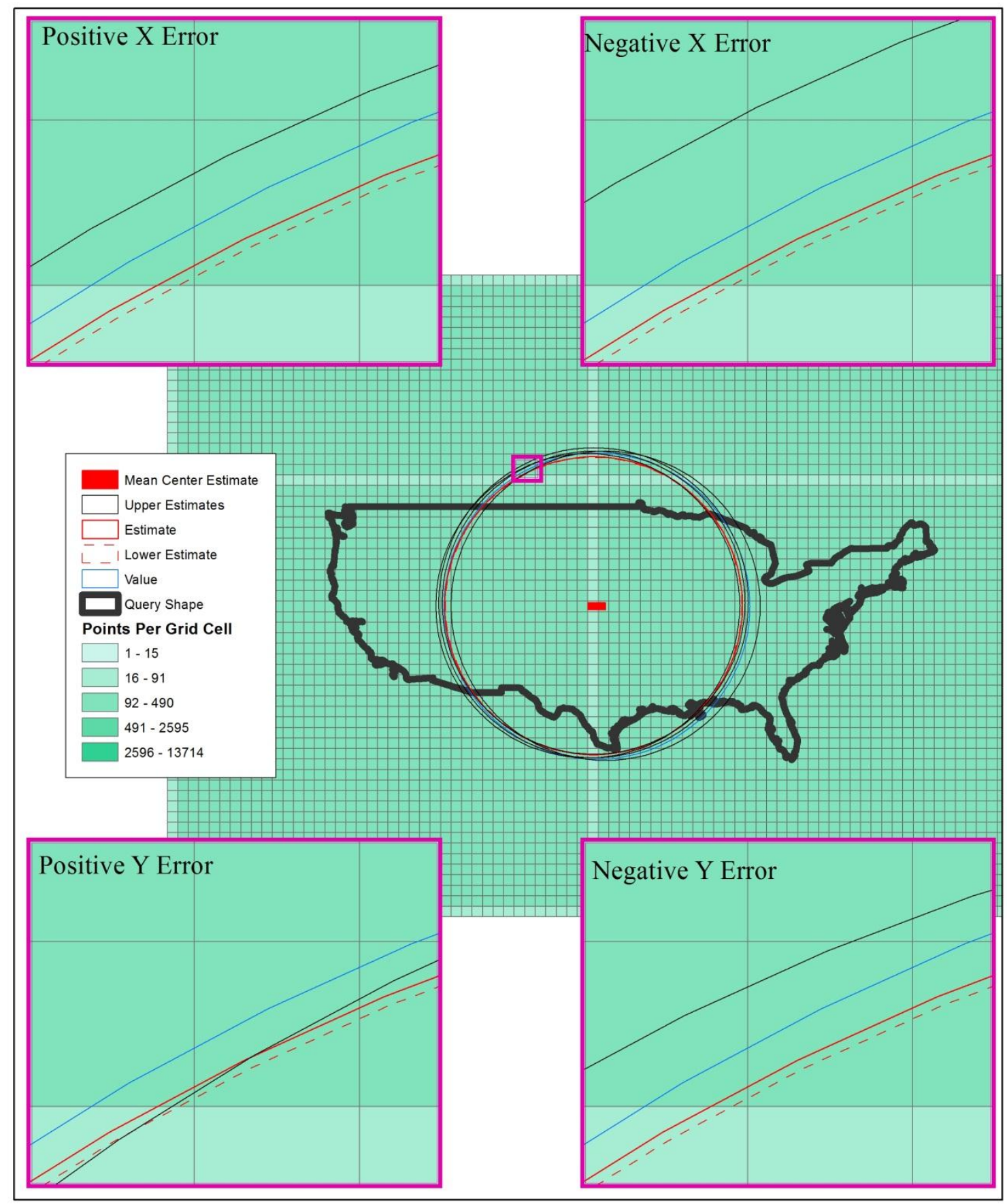

Figure 6-15. Error estimates of standard distance against the USA query shape and a uniform distribution 


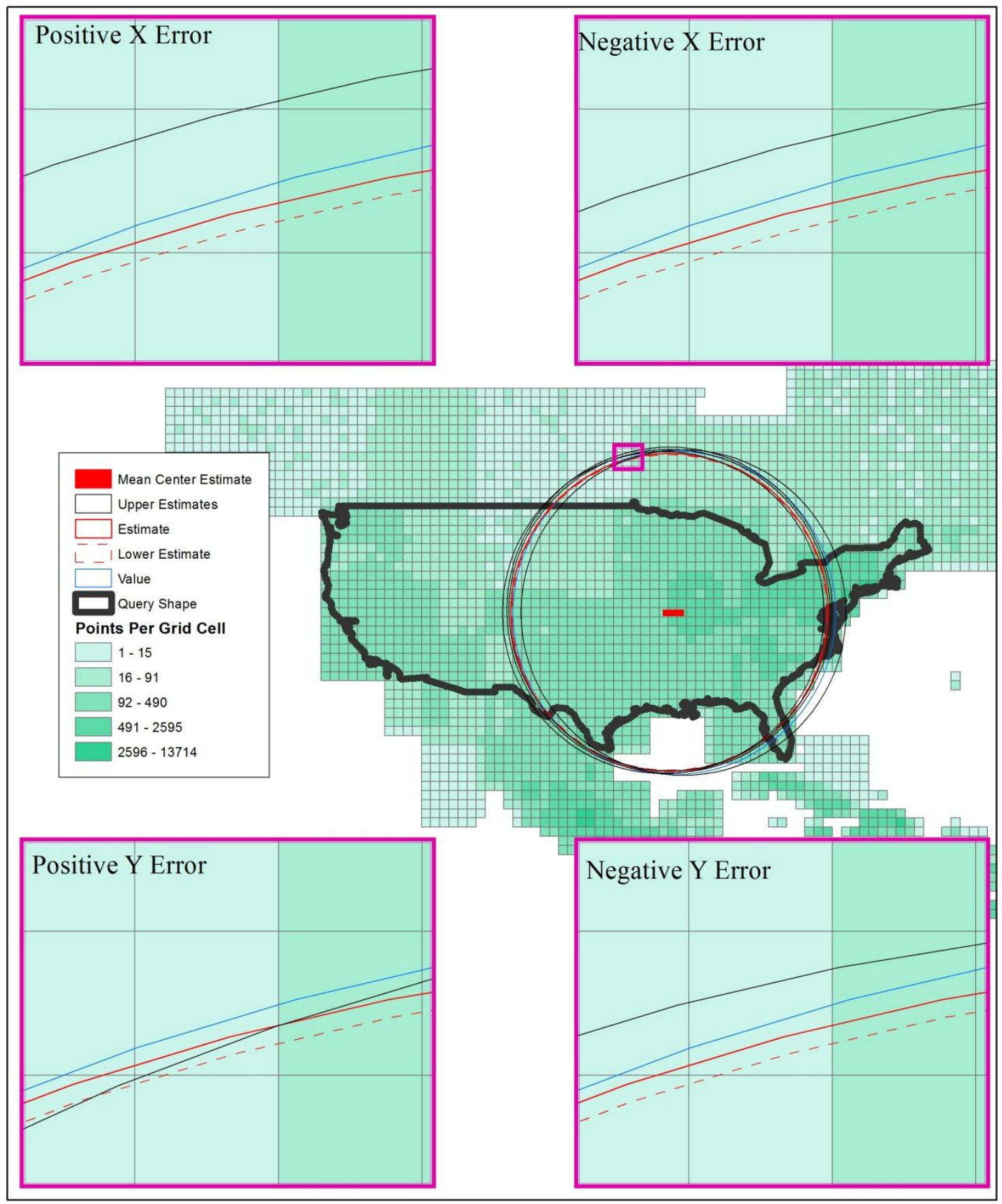

Figure 6-16. Error estimates of standard distance against the USA query shape and a clustered distribution 


\subsection{Conclusion}

This chapter shows that despite the limitations that Oracle has when implementing indexing through the Data Cartridge, it is possible to increase performance for specific operations by using indexing and purpose-build operators. This chapter has also demonstrated that the ideas conceived by this project are sound, producing accurate solutions, estimates, and estimation error limits. Finally, it demonstrates that extension of the SDE.ST_Geometry spatial index is possible with little modification to the underlying data type. This is a key point, as while the products that this project produced may never be used, the principles it demonstrates and the solutions that it provides can be used to implement a fully functional version of this architecture on a different database, or used to extend the SDE.ST_Geometry spatial index for other data intensive spatial metrics. 


\section{Chapter 7 - Conclusions and Future Work}

This projected attempted to increase the reputation of the SDE.ST_GEOMETRY spatial type in the Oracle user community, by demonstrating it is possible to build a purpose build domain index that allows users to increase their productivity. In that respect, the project has succeeded in producing a template that can be followed. This project failed to deliver a suitable metadata index structure due to the limitations of the Oracle Data Cartridge. Specifically, the inability of the domain index to identify when additional filters need to be applied to the result set. This limits the usefulness of the deliverables of this project as they have the possibility of returning incorrect result.

Future work could include investigation and porting of this system to other database management systems that may support index aware aggregate functions. Additional work to this implementation includes:

- Implementation of geodetic to three-dimensional coordinate conversion needed to calculate the mean center of a point population on the surface of the earth

- Numerical research into possible algorithms to calculate directional distributions of very large datasets without encountering floating-point errors

- Implementing additional operators that use the spatial index to increase the efficiency of spatial autocorrelation functions

The main premises that this project has operated under was that decreasing the amount of data needed to be read at the time of execution, would speed up the database system. In Chapter 6, this was shown to be true through the relationship between faster execution times verses logical and physical reads. This result proves that, in theory, optimizations could be introduced into database indexing structure that would increase aggregate operations across large volumes of point data. Technical limitations were encountered, but other databases may not share these limitations, and with additional research, fully functioning aggregates functions that resolve results directly from an index may be possible. 



\section{Works Cited}

Brimberg, J., Hansen, P., Mladenović, N., \& Taillard, E. D. (2000). Improvements and Comparison of Heuristics For Solving the Uncapacitated Multisource Weber Problem. Operations Research, 48(3), 444-460.

Coope, L. (1963). Location-Allocation Problems. Operations Research, 11(3), 331-343. ESRI. (2011a). Spatial Statistics toolbox licensing. Retrieved 2011, from ArcGIS Resource Center: http://help.arcgis.com/en/arcgisdesktop/10.0/help/index.html\#/Spatial_Statistics_t oolbox_licensing/005p00000048000000/

Esri. (2011b). The spatial grid index. Retrieved 2011, from ArcGIS Resource Center: http://help.arcgis.com/en/arcgisserver/10.0/help/arcgis_server_dotnet_help/index. html\#/The_spatial_grid_index/0093000015rq000000/

Fisher, N. I., Lewis, T., \& Embleton, B. (1987). Statistical Analysis of Spherical Data. Cambridge: The Press Syndicate of the University of Cambridge.

Furfey, P. H. (1927). A Note on Lefever's "Standard Deviational Ellipse". American Journal of Sociology, 33(1), 94-98.

Hammergren, T. C., \& Simon, A. R. (2009). Data Warehousing for Dummies. Hoboken, NJ: Wiley Publishing, Inc.

Huff, D. (1954). How to Lie with Statistics. New York: W.W. Norton \& Company, Inc. International Technology Group. (2011, 6). public.dhe.ibm.com. Retrieved 2 17, 2013, from IBM Support: ftp://public.dhe.ibm.com/software/data/swlibrary/infosphere/analyst-reports/ITG-ISAS-Exadata-Teradata.pdf

Klopp, R. (2012). Price/Performance of HANA, Exadata, Teradata, and Greenplum. Retrieved 02 17, 2013, from Database FOG Blog: http://robklopp.wordpress.com/2012/11/15/priceperformance-of-hana-exadatateradata-and-greenplum/

Kumler, M. P., \& Goodchild, M. F. (1992). The Population Center of Canada - Just North of Toronto?!? (D. G. Janelle, Ed.) Geographical snapshots of North America.

Lefever, D. W. (1926). Measuring Geographic Concentrations by Means of the Standard Deviational Ellipse. American Journal of Sociology, 32(1), 88-94.

Mohanty, A. (2012). www.slideshare.net. Retrieved 2 17, 2013, from Slide Share: http://www.slideshare.net/asismohanty/netezza-vs-teradata-vs-exadata

Oracle. (2010a). Oracle Database Concepts, $11 \mathrm{~g}$ Release 2 (11.2). Redwood City, California: Oracle USA, Inc.

Oracle. (2010b). SQL Language Reference. Redwood City, CA: Oracle.

O'Sullivan, D., \& Unwin, D. J. (2010). Geographic Information Analysis (2nd ed.). Hoboken: John Wiley \& Sons, Inc.

Rogerson, P. A. (2006). Statistical Methods for Geography: A Students Guide (2nd ed.). Thousand Oaks, California, USA: SAGE Publications Ltd.

Samet, H. (2006). Foundations of Multidimensional and Metric Data Structures. San Francisco: Morgan Kaufmann Publishers.

Wang, W., Yang, J., \& Muntz, R. (1997). STING : A Statistical Information Grid Approach to Spatial Data. VLDB '97 Proceedings of the 23rd International 
Conference on Very Large Data Bases (pp. 186-195). San Francisco: Morgan Kaufmann Publishers Inc.

Yuill, R. S. (1971). The Standard Deviational Ellipse: An Updated Tool for Spatial Description. Geografiska Annaler. Series B, Human Geography, 53(1), 28-39. 


\section{Appendix A. Files}

AGGR_MEAN_CENTER.sql

The AGGR_MEAN_CENTER.sql file creates the mean center aggregate function. This function is used for comparison purposes in the evaluation of the ST_MEAN_CENTER operator.

ClusterCreate.sql

This script creates four clustered datasets, containing each of $6,000,60,000,600,000$, and $6,000,000$ rows.

\section{MAKE.sql}

Use this file to install the ST_STAT_INDEX type, and all the associated operators. MAKE.sql is the file that creates and removes the relevant SQL packages from the system. This file performs the following actions:

1) Drops all the operators, packages and types associated with the statistical index

2) Recreates the index type

3) Recreates the operators, grants permissions on the operator to public

4) Recreates the aggregate operators

This script will only work if there are no indexes currently created. Otherwise, this script will fail.

RandomCreate.sql

This script creates four random datasets, containing each of $6,000,60,000,600,000$, and $6,000,000$ rows.

ST_DIR_DIST_TYPE.sql and ST_DIR_DIST.sql

These files define and create the package that calculates the directional distribution of a dataset.

Function CLEANUP returns NUMBER

- Cleans up any static or session based variables after the directional distribution has been calculated

Function DIR_DIST_GEOMETRY returns ST_GEOMETRY

- Returns a geometry that represents the result of the directional distribution calculation

\begin{tabular}{l|ll}
\hline Argument Name & Argument Type & Argument Direction \\
\hline SHAPE & ST_GEOMETRY & IN \\
FILTER & ST_GEOMETRY & IN \\
INDEXCTX & ODCIINDEXCTX & IN \\
SCANCTX & ST_STAT_INDEXTYPE_IM & IN/OUT \\
SCANFLG & NUMBER & IN \\
\hline
\end{tabular}


Function DIR_DIST_GEOMETRY returns ST_GEOMETRY

- Returns a geometry that represents the result of the directional distribution calculation, with the solve method specified

\begin{tabular}{l|ll}
\hline Argument Name & Argument Type & Argument Direction \\
\hline SHAPE & ST_GEOMETRY & IN \\
FILTER & ST_GEOMETRY & IN \\
METHOD & NUMBER & IN \\
INDEXCTX & ODCIINDEXCTX & IN \\
SCANCTX & ST_STAT_INDEXTYPE_IM & IN/OUT \\
SCANFLG & NUMBER & IN \\
\hline
\end{tabular}

Function DIR_DIST_MAJOR_FUNCTION returns NUMBER

- Returns a number that represents the length of the directional distribution calculation's major axis

\begin{tabular}{l|ll}
\hline Argument Name & Argument Type & Argument Direction \\
\hline SHAPE & ST_GEOMETRY & IN \\
FILTER & ST_GEOMETRY & IN \\
INDEXCTX & ODCIINDEXCTX & IN \\
SCANCTX & ST_STAT_INDEXTYPE_IM & IN/OUT \\
SCANFLG & NUMBER & IN \\
\hline
\end{tabular}

Function DIR_DIST_MAJOR_FUNCTION returns NUMBER

- Returns a geometry that represents the result of the directional distribution calculation, with the solve method specified

\begin{tabular}{l|ll}
\hline Argument Name & Argument Type & Argument Direction \\
\hline SHAPE & ST_GEOMETRY & IN \\
FILTER & ST_GEOMETRY & IN \\
METHOD & NUMBER & IN \\
INDEXCTX & ODCIINDEXCTX & IN \\
SCANCTX & ST_STAT_INDEXTYPE_IM & IN/OUT \\
SCANFLG & NUMBER & IN \\
\hline
\end{tabular}

Function DIR_DIST_MINOR_FUNCTION returns NUMBER

- Returns a geometry that represents the length of the directional distribution calculation's minor axis

\begin{tabular}{l|ll}
\hline Argument Name & Argument Type & Argument Direction \\
\hline SHAPE & ST_GEOMETRY & IN \\
FILTER & ST_GEOMETRY & IN \\
INDEXCTX & ODCINDEXCTX & IN \\
SCANCTX & ST_STAT_INDEXTYPE_IM & IN/OUT \\
SCANFLG & NUMBER & IN \\
\hline
\end{tabular}

Function DIR_DIST_MINOR_FUNCTION returns NUMBER 
- Returns a geometry that represents the result of the directional distribution calculation, with the solve method specified

\begin{tabular}{l|ll}
\hline Argument Name & Argument Type & Argument Direction \\
\hline SHAPE & ST_GEOMETRY & IN \\
FILTER & ST_GEOMETRY & IN \\
METHOD & NUMBER & IN \\
INDEXCTX & ODCIINDEXCTX & IN \\
SCANCTX & ST_STAT_INDEXTYPE_IM & IN/OUT \\
SCANFLG & NUMBER & IN \\
\hline
\end{tabular}

Function DIR_DIST_THETA_FUNCTION returns NUMBER

- Returns a geometry that represents the result of the directional distribution calculation's theta angle

\begin{tabular}{l|ll}
\hline Argument Name & Argument Type & Argument Direction \\
\hline SHAPE & ST_GEOMETRY & IN \\
FILTER & ST_GEOMETRY & IN \\
INDEXCTX & ODCIINDEXCTX & IN \\
SCANCTX & ST_STAT_INDEXTYPE_IM & IN/OUT \\
SCANFLG & NUMBER & IN \\
\hline
\end{tabular}

Function DIR_DIST_THETA_FUNCTION returns NUMBER

- Returns a geometry that represents the result of the directional distribution calculation, with the solve method specified

\begin{tabular}{l|ll}
\hline Argument Name & Argument Type & Argument Direction \\
\hline SHAPE & ST_GEOMETRY & IN \\
FILTER & ST_GEOMETRY & IN \\
METHOD & NUMBER & IN \\
INDEXCTX & ODCIINDEXCTX & IN \\
SCANCTX & ST_STAT_INDEXTYPE_IM & IN/OUT \\
SCANFLG & NUMBER & IN \\
\hline
\end{tabular}

Function GET_OBJECT returns NUMBER

- The core function for calculating directional distribution. On the first execution, this function will calculate the distribution, and set the value in the INDEXCTX parameter. Subsequent executions will return the previously calculated value.

\begin{tabular}{l|ll}
\hline Argument Name & Argument Type & Argument Direction \\
\hline FILTER & ST_GEOMETRY & IN \\
INDEXCTX & ODCIINDEXCTX & IN \\
SCANCTX & ST_STAT_INDEXTYPE_IM & IN/OUT \\
SCANFLG & NUMBER & IN \\
NEEDESTIMATE & NUMBER & IN \\
\hline
\end{tabular}

ST_MEAN_CENTER_TYPE.sql and ST_MEAN_CENTER.sql

These packages define and create the functionality that supports mean center calculations. 
ST_MEDIAN_CENTER_TYPE.sql and ST_MEDIAN_CENTER.sql

These packages define and create the functionality that supports median center calculations.

ST_STAT_INDEX_TYPE.sql and ST_STAT_INDEX.sql

The behavior of the statistical index is defined within this package. This is the index context object that is provided to the auxiliary operators, it contains fields for storing the different operator results. It also contains the Oracle defined methods that must be implemented in order to support domain index operations.

\section{ST_STAT_INDEX_OPERATORS.sql}

This files implements functions that are called if one of the ST_STAT_INDEX filter operators are called on a spatial column that does not have a ST_STAT_INDEX built on it.

\section{ST_STAT_UTIL_TYPE.sql and ST_STAT_UTIL.sql}

Oracle has limitations on the types of objects that can be stored in object types (insatiable type). Custom types and PL/SQL tables are not allowed to be members of an object type. For this reason, many of the data structures that are used by the auxiliary operators are stored in this package. There are also extra costs associated with accessing non-local package properties, so much of the heavy processing and aggregation values are also done in this package.

ST_STD_DIST_TYPE.sql and ST_STD_DIST.sql

These packages define and create the functionality that supports standard distance calculations.

\section{UniformCreate.sql}

This script creates four uniform datasets, containing each of approximately $6,000,60,000$, 600,000 , and 6,000,000 rows. 


\section{Appendix B. Operators}

\section{Mean Center Auxiliary Operators}

Each operator calculates the mean center of the indexed population through the methods outlined in Section 4.4.1.

Ancillary operator to:

ST_STAT_CONTAINS(SDE.ST_GEOMETRY,SSE.ST_GEOMETRY), ST_STAT_CONTAINS(SDE.ST_GEOMETRY,SDE.ST_GEOMETRY, VARCHAR2)

Example:

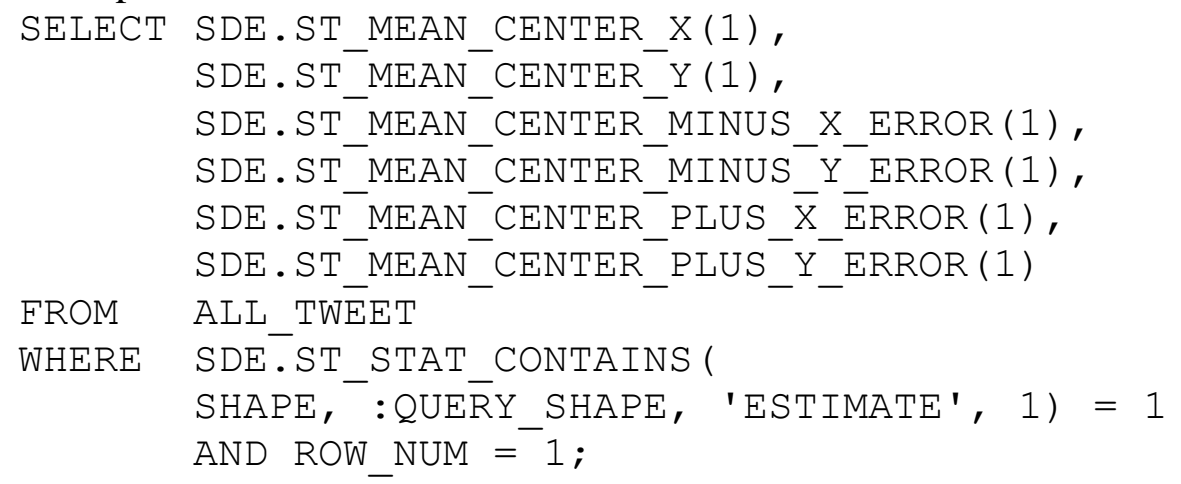

MEAN_CENTER

Returns: SDE.ST_GEOMETRY point at the calculated mean center

Defined by: MEAN_CENTER.MEAN_CENTER_FUNCTION;

ST_MEAN_CENTER_X

Returns: NUMBER equal to the $\mathrm{X}$ value of the calculated mean center

Defined by: MEAN_CENTER.MEAN_CENTER_FUNCTION_X

\section{ST_MEAN_CENTER_Y}

Returns: NUMBER equal to the Y value of the calculated mean center

Defined by: MEAN_CENTER.MEAN_CENTER_FUNCTION_Y

\section{ST_MEAN_CENTER_PLUS_X_ERROR}

Returns: NUMBER equal to the positive X error of the calculated mean center Defined by: MEAN_CENTER.ERROR_PLUS_X

ST_MEAN_CENTER_PLUS_Y_ERROR

Returns: NUMBER equal to the positive Y error of the calculated mean center Defined by: MEAN_CENTER.ERROR_PLUS_Y 
Returns: NUMBER equal to the negative $X$ error of the calculated mean center Defined by: MEAN_CENTER.ERROR_MINUS_X;

ST_MEAN_CENTER_MINUS_Y_ERROR

Returns: NUMBER equal to the negative Y error of the calculated mean center Defined by: MEAN_CENTER.ERROR_MINUS_Y

\section{Median Center Auxiliary Operators}

Each operator calculates the median center of the indexed population through the methods outlined in Section 4.4.2.

Ancillary operator to:

ST_STAT_CONTAINS(SDE.ST_GEOMETRY, SDE.ST_GEOMETRY), ST_STAT_CONTAINS(SDE.ST_GEOMETRY,SDE.ST_GEOMETRY, VARCHAR2)

Example:

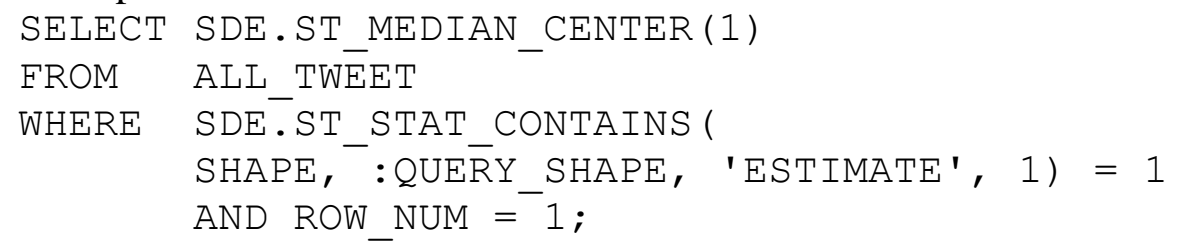

MEDIAN_CENTER

Returns: SDE.ST_GEOMETRY point at the calculated median center

Defined by: MEDIAN_CENTER.MEDIAN_CENTER_FUNCTION

\section{ST_MEDIAN_CENTER_X}

Returns: NUMBER equal to the $X$ value of the calculated median center Defined by: MEDIAN_CENTER.MEDIAN_CENTER_FUNCTION_X

\section{ST_MEDIAN_CENTER_Y}

Returns: NUMBER equal to the $Y$ value of the calculated median center Defined by: MEDIAN_CENTER.MEDIAN_CENTER_FUNCTION_Y

\section{ST_MEDIAN_CENTER_PLUS_X_ERROR}

Returns: NUMBER equal to the positive X error of the calculated median center Defined by: MEDIAN_CENTER.ERROR_PLUS_X

\section{ST_MEDIAN_CENTER_PLUS_Y_ERROR}

Returns: NUMBER equal to the positive Y error of the calculated median center Defined by: MEDIAN_CENTER.ERROR_PLUS_Y

\section{ST_MEDIAN_CENTER_MINUS_X_ERROR}

Returns: NUMBER equal to the negative $X$ error of the calculated median center 
Defined by: MEDIAN_CENTER.ERROR_MINUS_X

\section{ST_MEDIAN_CENTER_MINUS_Y_ERROR}

Returns: NUMBER equal to the negative $Y$ error of the calculated median center Defined by: MEDIAN_CENTER.ERROR_MINUS_Y

\section{Standard Distance Auxiliary Operators}

Each operator calculates the standard distance of the indexed population through the methods outlined in Section 4.4.3.

Ancillary operator to:

ST_STAT_CONTAINS(SDE.ST_GEOMETRY,SDE.ST_GEOMETRY),

ST_STAT_CONTAINS(SDE.ST_GEOMETRY, SDE.ST_GEOMETRY, VARCHAR2)

Example:

SELECT SDE.ST_STANDARD_DISTANCE (1)

FROM ALL TWEET

WHERE SDE.ST_STAT_CONTAINS(

SHAPE, :QUERY_SHAPE, 'SOLVE', 1) $=1$

AND ROW_NUM $=1$;

\section{ST_STANDARD_DISTANCE}

Returns: NUMBER equal to the calculated standard distance

Defined by: STD_DIST.STD_DIST_FUNCTION

\section{ST_STD_DIST_GEOMETRY}

Returns: ST_GEOMETRY equal to the calculated standard circle

Defined by: STD_DIST.STD_DIST_GEOMETRY

\section{ST_STD_DIST_PLUS_ERROR}

Returns: NUMBER equal to the error of the calculated standard distance at the estimated mean center

Defined by: STD_DIST.ERROR_PLUS

\section{ST_STD_DIST_PLUS_ERROR_UL}

Returns: NUMBER equal to the error of the calculated standard distance at the estimated upper left limit of the mean center Defined by: STD_DIST.ERROR_PLUS_UL

\section{ST_STD_DIST_PLUS_ERROR_UR}

Returns: NUMBER equal to the error of the calculated standard distance at the estimated upper right limit of the mean center Defined by: STD_DIST.ERROR_PLUS_UR 
ST_STD_DIST_PLUS_ERROR_LL

Returns: NUMBER equal to the error of the calculated standard distance at the estimated lower left limit of the mean center

Defined by: STD_DIST.ERROR_PLUS_LL

\section{ST_STD_DIST_PLUS_ERROR_LR}

Returns: NUMBER equal to the error of the calculated standard distance at the estimated lower right limit of the mean center

Defined by: STD_DIST.ERROR_PLUS_LR

\section{ST_STD_DIST_MINUS_ERROR}

Returns: NUMBER equal to the negative error of the calculated standard distance at the estimated mean center

Defined by: STD_DIST.ERROR_MINUS

\section{Directional Distribution Auxiliary Operators}

Each operator calculates the directional distribution of the indexed population through the methods outlined in Section 4.4.4. These operators will fail when run on large populations.

Ancillary operator to:

ST_STAT_CONTAINS(SDE.ST_GEOMETRY, SDE.ST_GEOMETRY), ST_STAT_CONTAINS(SDE.ST_GEOMETRY, SDE.ST_GEOMETRY, VARCHAR2)

Example:

SELECT SDE.ST_DIR_DIST_GEOMETRY (1)

FROM ALL TWEET

WHERE SDE.ST_STAT_CONTAINS (

SHAPE, :QUERY_SHAPE, 'SOLVE', 1) $=1$

AND ROW_NUM $=-1$;

\section{ST_DIR_DISTRIBUTION_MAJOR}

Returns: NUMBER equal to the calculated major axis of the directional distribution Defined by: DIR_DIST.DIR_DIST_MAJOR_FUNCTION

\section{ST_DIR_DISTRIBUTION_MINOR}

Returns: NUMBER equal to the calculated minor axis of the directional distribution Defined by: DIR_DIST.DIR_DIST_MINOR_FUNCTION

\section{ST_DIR_DISTRIBUTION_THETA}

Returns: NUMBER equal to the calculated angle of the major axis of the directional distribution

Defined by: DIR_DIST.DIR_DIST_THETA_FUNCTION 


\section{ST_DIR_DIST_GEOMETRY}

Returns: NUMBER equal to the calculated major axis of the directional distribution Defined by: DIR_DIST.DIR_DIST_GEOMETRY 


\section{Appendix C. Performance Metrics}

The Oracle performance metrics are defined in the Oracle documentation, specifically the Oracle 11g Database Reference, Appendix E - Statistics Description.

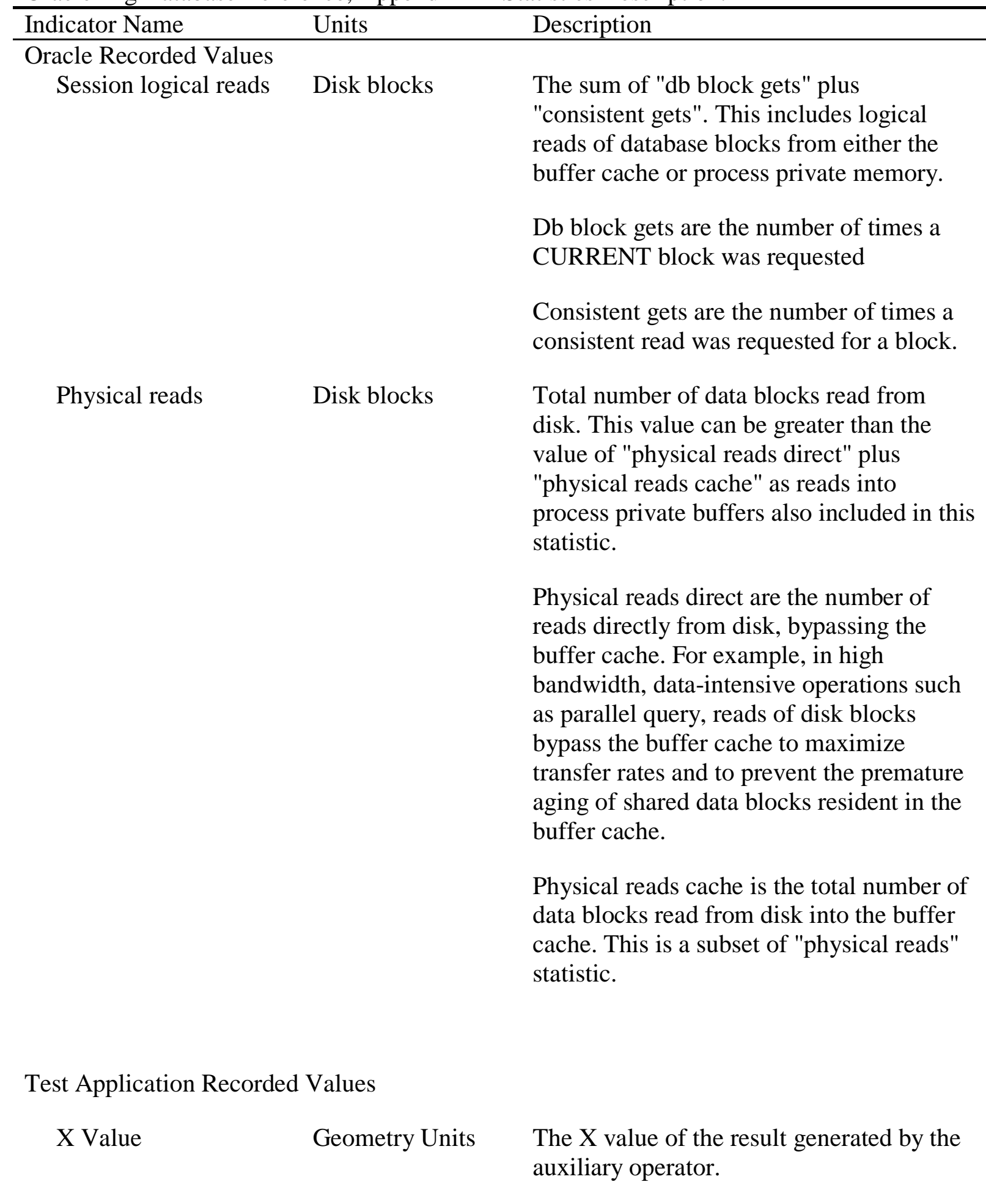




\begin{tabular}{|c|c|c|}
\hline Y Value & Geometry Units & $\begin{array}{l}\text { The Y value of the result generated by the } \\
\text { auxiliary operator. }\end{array}$ \\
\hline $\mathrm{X}$ Variation & Geometry Units & $\begin{array}{l}\text { The difference between the } X \text { value and the } \\
\text { expected } X \text { value }\end{array}$ \\
\hline Y Variation & Geometry Units & $\begin{array}{l}\text { The difference between the } \mathrm{Y} \text { value and the } \\
\text { expected } \mathrm{Y} \text { value }\end{array}$ \\
\hline Net stopwatch & Seconds & $\begin{array}{l}\text { The time elapsed in the test application. } \\
\text { This is calculated by using time stamps in } \\
\text { the test application. This accounts for time } \\
\text { that the tread waited in a blocked state. }\end{array}$ \\
\hline SQL Error & Boolean & $\begin{array}{l}1 \text { if there was an error in the test } \\
\text { application or SQL statement; otherwise } 0 \text {. }\end{array}$ \\
\hline Pos X Expected Error & Geometry Units & $\begin{array}{l}\text { The amount of error in the positive } \mathrm{X} \\
\text { direction that was generated by the } \\
\text { auxiliary operator estimate. }\end{array}$ \\
\hline Pos Y Expected Error & Geometry Units & $\begin{array}{l}\text { The amount of error in the positive } Y \\
\text { direction that was generated by the } \\
\text { auxiliary operator estimate. }\end{array}$ \\
\hline Neg X Expected Error & Geometry Units & $\begin{array}{l}\text { The amount of error in the negative } X \\
\text { direction that was generated by the } \\
\text { auxiliary operator estimate. }\end{array}$ \\
\hline Neg Y Expected Error & Geometry Units & $\begin{array}{l}\text { The amount of error in the negative } Y \\
\text { direction that was generated by the } \\
\text { auxiliary operator estimate. }\end{array}$ \\
\hline
\end{tabular}




\section{Appendix D. Test Data}

The test data that was used to performance test this project was created using three scripts. The scripts, UniformCreate.sql, RandomCreate.sql, and UniformCreate.sql generate four tables each. These tables are listed in the table below, with metadata values listed for each table. These metadata values are:

- $\%$ Free - The amount of space that the DBMS will leave empty in each block.

- Row Count - The number of rows in the table

- Row Chained - The number of rows that are stored in more than one block.

- Row length - The average length of the row, in bytes.

- Block Count - The number of database blocks

- Block Empty - The number of empty blocks.

- Block Free - The number of blocks that are on the table's free list.

\begin{tabular}{|c|c|c|c|c|c|c|c|}
\hline \multirow[t]{2}{*}{ Name } & \multirow[t]{2}{*}{$\%$ Free } & \multicolumn{3}{|c|}{ Row } & \multicolumn{3}{|c|}{ Block } \\
\hline & & Count & Chained & Length & Count & Empty & Free \\
\hline RANDOMDIST_6K & 10 & 6000 & 0 & 182 & 244 & 0 & 0 \\
\hline RANDOMDIST_60K & 10 & 60000 & 0 & 183 & 1378 & 0 & 0 \\
\hline RANDOMDIST_600K & 10 & 600000 & 0 & 183 & 14177 & 0 & 0 \\
\hline RANDOMDIST_6000K & 10 & 6000000 & 0 & 184 & 138644 & 0 & 0 \\
\hline UNIFORMDIST_6K & 10 & 6000 & 0 & 138 & 103 & 0 & 0 \\
\hline UNIFORMDIST_60K & 10 & 60230 & 0 & 183 & 1378 & 0 & 0 \\
\hline UNIFORMDIST_600K & 10 & 600000 & 0 & 145 & 11117 & 0 & 0 \\
\hline UNIFORMDIST_6000K & 10 & 6003374 & 0 & 184 & 138644 & 0 & 0 \\
\hline CLUSTERDIST_'̄ & 10 & 6018 & 0 & 182 & 244 & 0 & 0 \\
\hline CLUSTERDIST_60K & 10 & 60191 & 0 & 183 & 1378 & 0 & 0 \\
\hline CLUSTERDIST_600K & 10 & 601921 & 0 & 183 & 14177 & 0 & 0 \\
\hline CLUSTERDIST_6000K & 10 & 6019198 & 0 & 184 & 138644 & 0 & 0 \\
\hline
\end{tabular}




\section{Appendix E. Additional Figures}

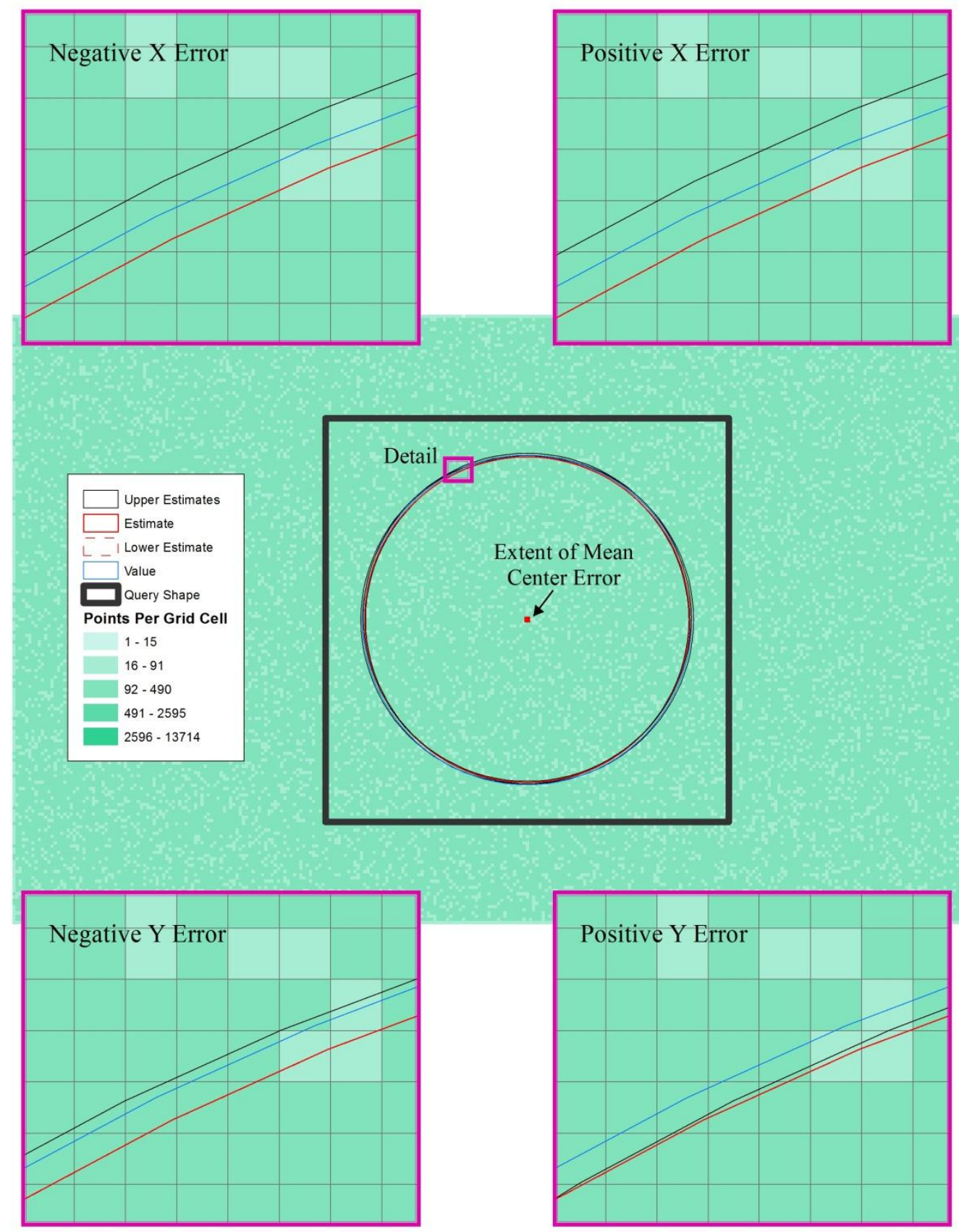

Figure E-1. Error estimates of standard distance against the Square query shape and a random distribution 

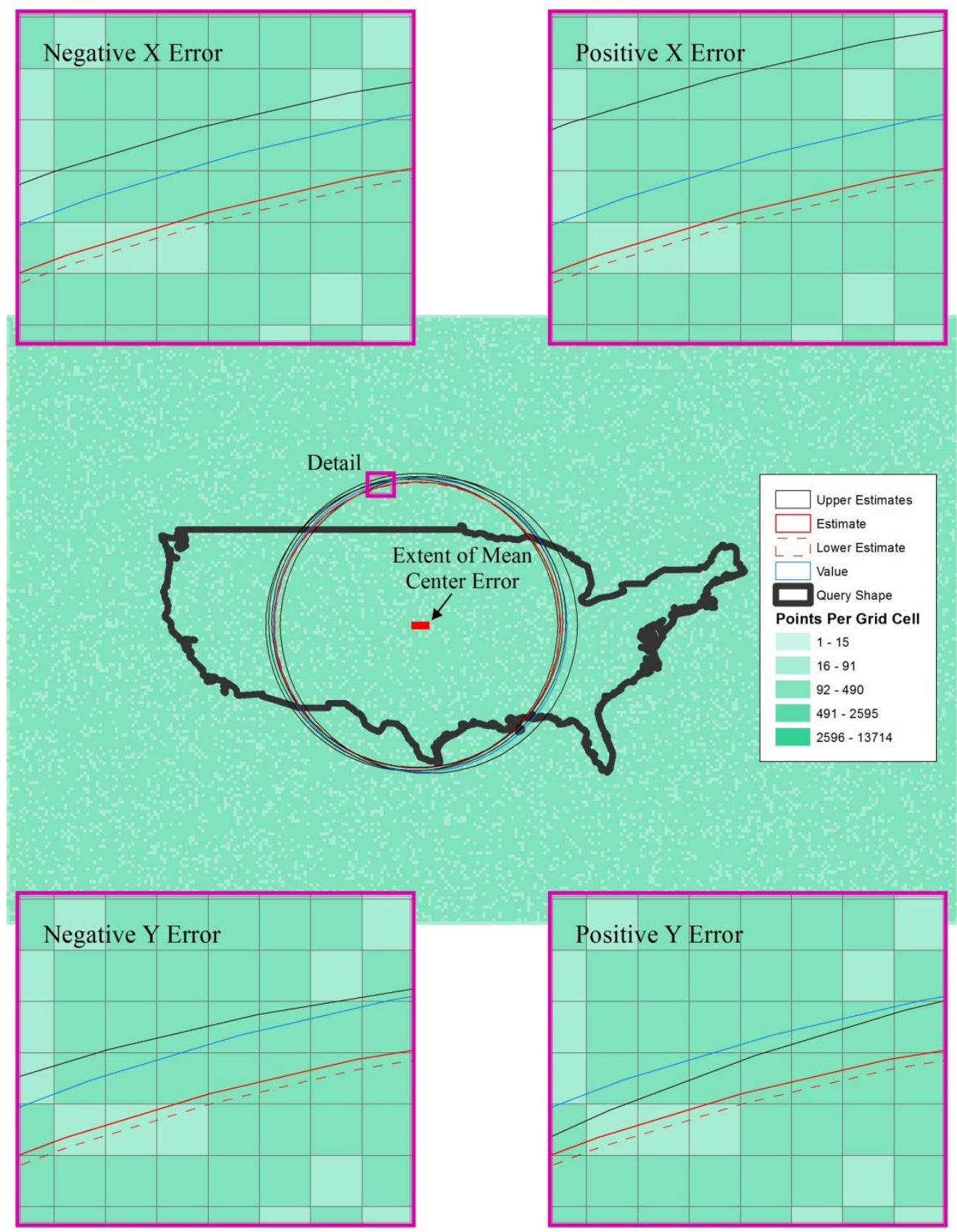

Figure E-2. Error estimates of standard distance against the USA query shape and a random distribution 


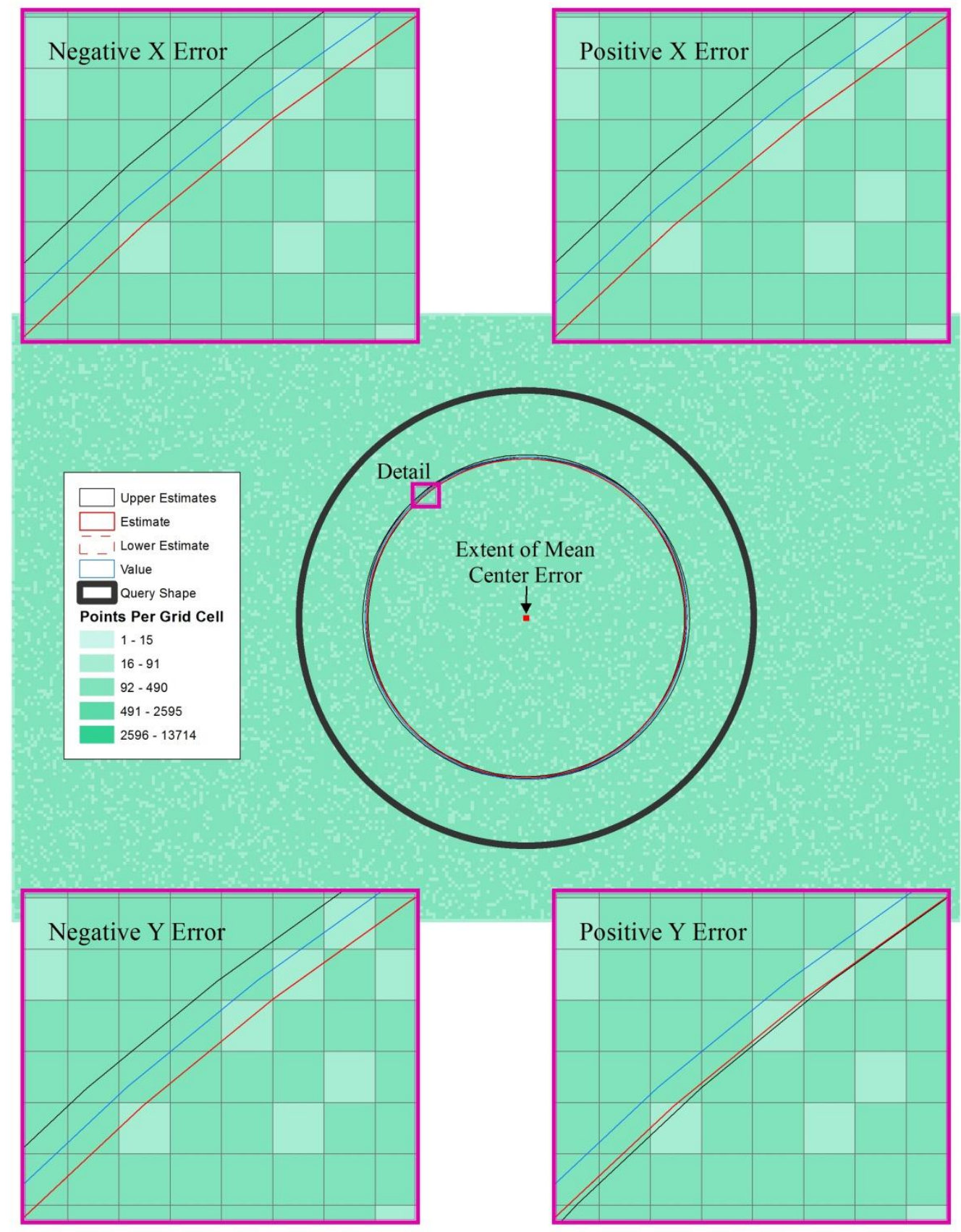

Figure E-3. Error estimates of standard distance against the Circle query shape and a random distribution 


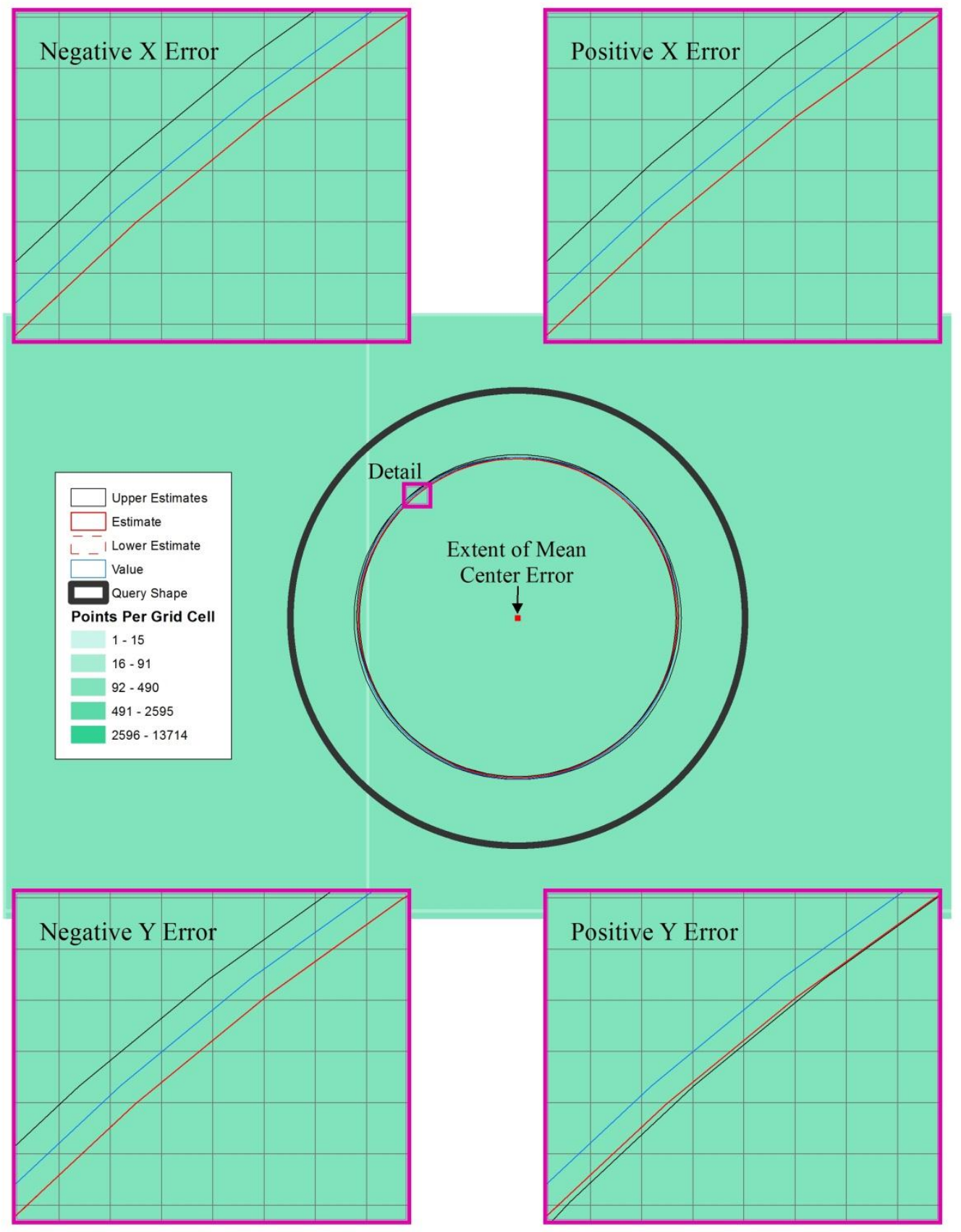

Figure E-4. Error estimates of standard distance against the Circle query shape and a uniform distribution 

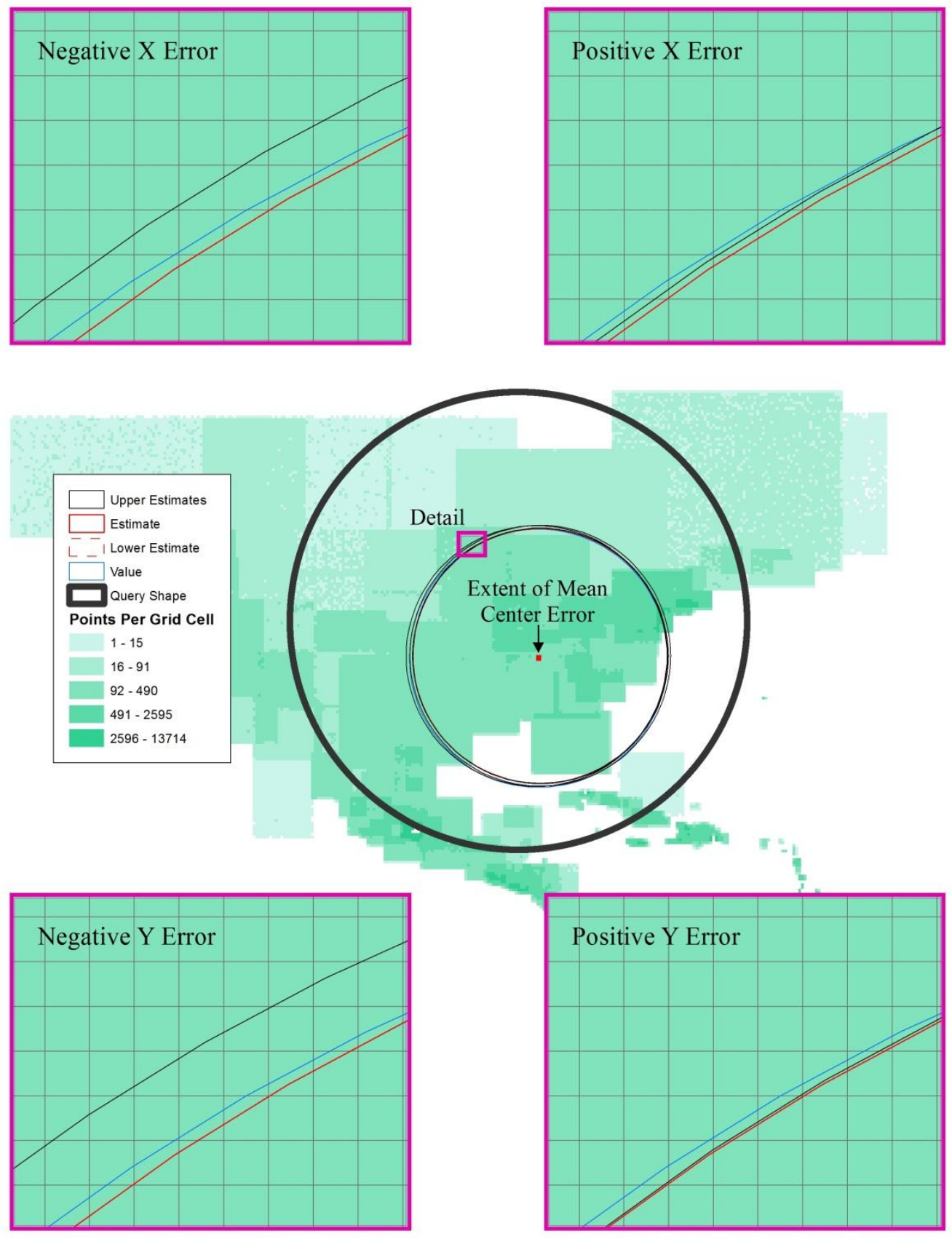

Figure E-5. Error estimates of standard distance against the Circle query shape and a clustered distribution 


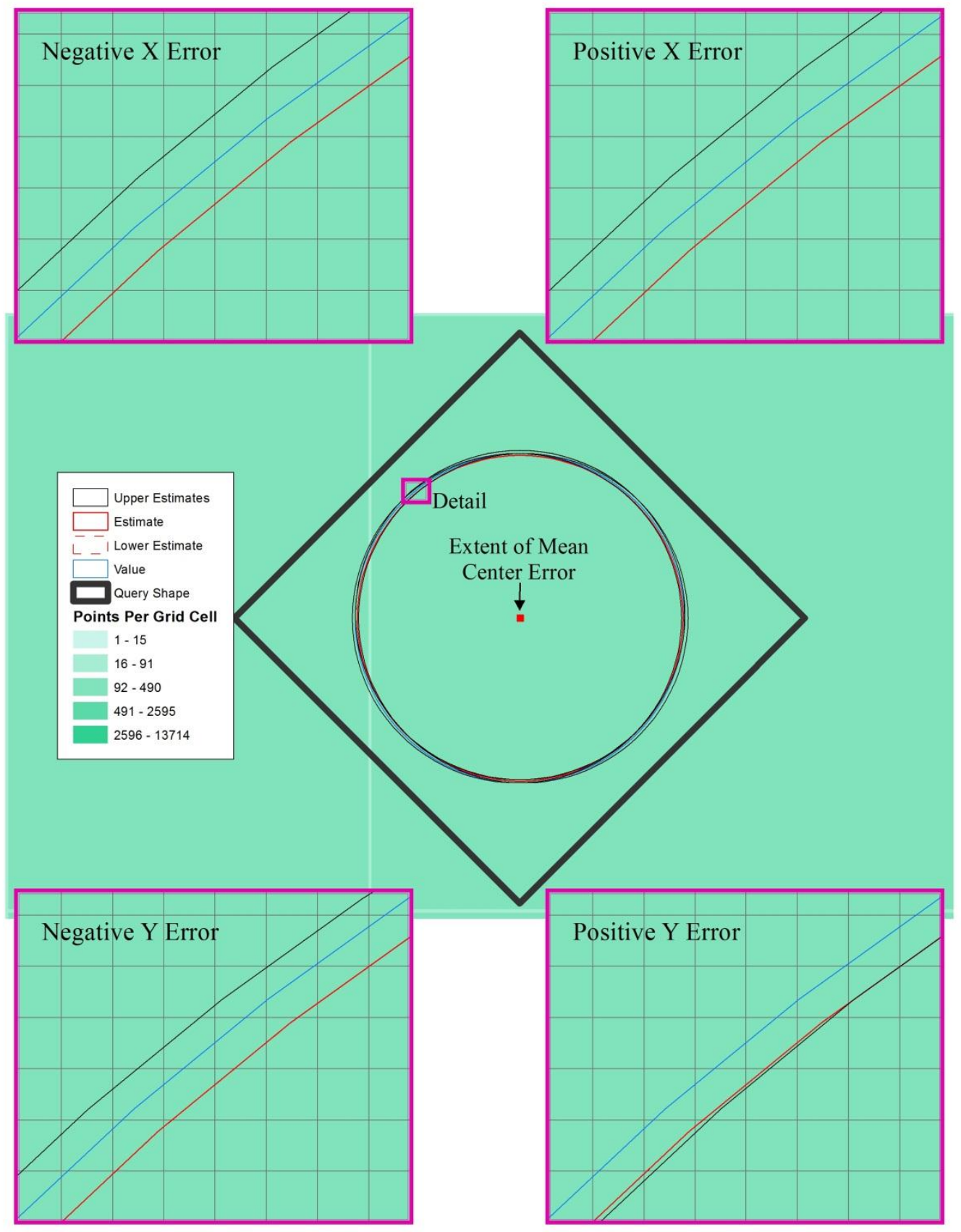

Figure E-6. Error estimates of standard distance against the Diamond query shape and a random distribution 


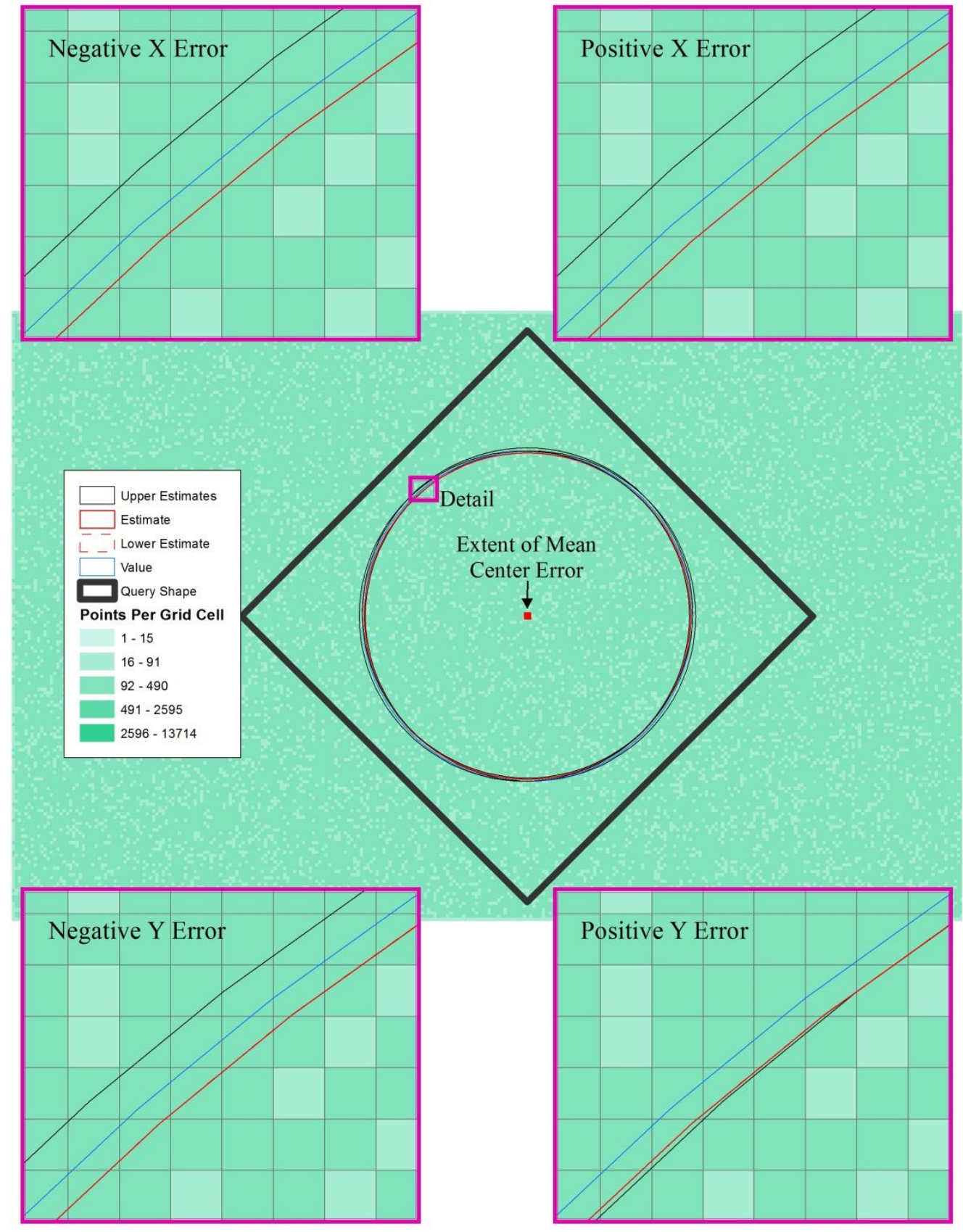

Figure E-7. Error estimates of standard distance against the Diamond query shape and a uniform distribution 


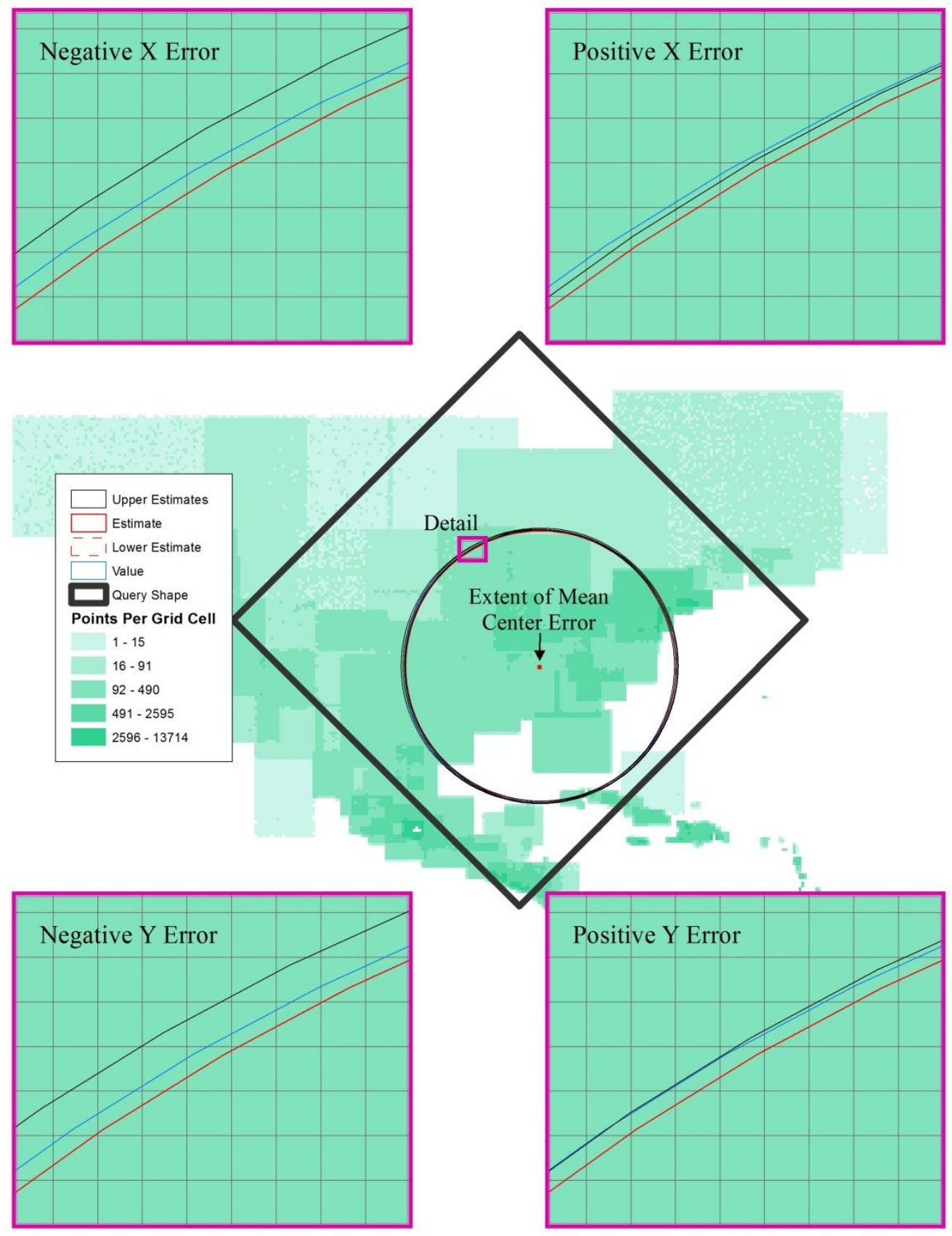

Figure E-8. Error estimates of standard distance against the Diamond query shape and a clustered distribution 


\section{Appendix F. User Permissions}

The following are the permissions that were granted to each user before the PL/SQL packages were installed.

grant CREATE SESSION TO SDE;

grant CREATE TABLE TO SDE;

grant CREATE TRIGGER TO SDE;

grant CREATE VIEW TO SDE;

grant CREATE PROCEDURE TO SDE;

grant CREATE SEQUENCE TO SDE;

grant EXECUTE ON DBMS_CRYPTO TO SDE;

grant CREATE INDEXTYPE TO SDE;

grant CREATE LIBRARY TO SDE;

grant CREATE OPERATOR TO SDE;

grant CREATE PUBLIC SYNONYM TO SDE;

grant CREATE TYPE TO SDE;

grant DROP PUBLIC SYNONYM TO SDE;

grant ALTER ANY INDEX TO SDE;

grant CREATE ANY INDEX TO SDE;

grant CREATE ANY TRIGGER TO SDE;

grant CREATE ANY VIEW TO SDE;

grant DROP ANY INDEX TO SDE;

grant DROP ANY VIEW TO SDE;

grant SELECT ANY TABLE TO SDE;

grant UPDATE ANY TABLE TO SDE;

grant DELETE ANY TABLE TO SDE;

grant INSERT ANY TABLE TO SDE;

grant ADMINISTER DATABASE TRIGGER TO SDE;

grant UNLIMITED TABLESPACE to SDE;

Create Data Owner:

grant CREATE SESSION TO map;

grant CREATE TABLE TO map;

grant CREATE TRIGGER TO map;

grant CREATE VIEW TO map;

grant CREATE PROCEDURE TO map;

grant CREATE SEQUENCE TO map;

grant UNLIMITED TABLESPACE TO map; 


\section{Appendix G. Equation Manipulation}

Rearrangement of Equation 2.8 to Equation 2.9.

$$
\begin{aligned}
& s_{d}=\sqrt{\frac{\sum_{i=1}^{n}\left(d_{i c}\right)^{2}}{n}} \\
& s_{d}=\sqrt{\frac{\sum_{i=1}^{n}\left(\sqrt{\left(x_{i}-\bar{x}\right)^{2}+\left(y_{i}-\bar{y}\right)^{2}}\right)^{2}}{n}} \\
& s_{d}=\sqrt{\frac{\sum_{i=1}^{n}\left(x^{2}-2 x \bar{x}+\bar{x}^{2}+y^{2}-2 y \bar{y}+\bar{y}^{2}\right)}{n}} \\
& s_{d}=\sqrt{\frac{\sum_{i=1}^{n}\left(x^{2}+y^{2}\right)+\sum_{i=1}^{n}\left(\bar{x}^{2}-2 x \bar{x}\right)+\sum_{i=1}^{n}\left(\bar{y}^{2}-2 y \bar{y}\right)}{n}} \\
& s_{d}=\sqrt{\frac{\sum_{i=1}^{n} x^{2}+\sum_{i=1}^{n} y^{2}}{n}+\frac{n \bar{x}^{2}-2 \bar{x} \sum_{i=1}^{n} x}{n}+\frac{n \bar{y}^{2}-2 \bar{y} \sum_{i=1}^{n} y}{n}} \\
& s_{d}=\sqrt{\frac{\sum_{i=1}^{n} x^{2}+\sum_{i=1}^{n} y^{2}+\sum_{i=1}^{n} y^{2}}{n}+\frac{n \bar{x}^{2}}{n}-2 \bar{x} \frac{\sum_{i=1}^{n} x}{n}+\frac{n \bar{y}^{2}}{n}-2 \bar{y} \frac{\sum_{i=1}^{n} y}{n}} \\
& s_{d}=\sqrt{\frac{\sum_{i=1}^{n} x^{2}+\sum_{i=1}^{n} y^{2}}{n}-\bar{x}^{2}-\bar{y}^{2}-2 \bar{y}^{2}}
\end{aligned}
$$

Rearrangement of Equation 2.10 and 2.11 for single pass calculation

$$
\begin{aligned}
& s_{d x}=\sqrt{\frac{\sum_{i=1}^{n}\left(x_{i}-\bar{x}\right)^{2}}{n}, \quad s_{d y}=\sqrt{\frac{\sum_{i=1}^{n}\left(y_{i}-\bar{y}\right)^{2}}{n}}} \\
& s_{d x}=\sqrt{\frac{\sum_{i=1}^{n}\left(x^{2}-2 x \bar{x}+\bar{x}^{2}\right)}{n}}, \quad s_{d y}=\sqrt{\frac{\sum_{i=1}^{n}\left(y^{2}-2 y \bar{y}+\bar{y}^{2}\right)}{n}} \\
& s_{d x}=\sqrt{\frac{\sum_{i=1}^{n}\left(x^{2}\right)-2 \bar{x} \sum_{i=1}^{n}(x)}{n}+\bar{x}^{2}}, \quad s_{d y}=\sqrt{\frac{\sum_{i=1}^{n}\left(y^{2}\right)-2 \bar{x} \sum_{i=1}^{n}(y)}{n}+\bar{y}^{2}} \\
& s_{d x}=\sqrt{\frac{\sum_{i=1}^{n}\left(x^{2}\right)}{n}-2 \bar{x}^{2}+\bar{x}^{2}}, \quad s_{d y}=\sqrt{\frac{\sum_{i=1}^{n}\left(y^{2}\right)}{n}-2 \bar{y}^{2}+\bar{y}^{2}} \\
& s_{d x}=\sqrt{\frac{\sum_{i=1}^{n}\left(x^{2}\right)}{n}-\bar{x}^{2}}, \quad s_{d y}=\sqrt{\frac{\sum_{i=1}^{n}\left(y^{2}\right)}{n}-\bar{y}^{2}}
\end{aligned}
$$

Rearrangement of Equation 2.12 and 2.13 for single pass calculation 


$$
\begin{aligned}
& s_{d x}=\sqrt{\frac{\sum_{i=1}^{n}\left(\left(x_{i}-\bar{x}\right) \cos \theta-\left(y_{i}-\bar{y}\right) \sin \theta\right)^{2}}{n}}, \quad s_{d y}=\sqrt{\frac{\sum_{i=1}^{n}\left(\left(x_{i}-\bar{x}\right) \sin \theta-\left(y_{i}-\bar{y}\right) \cos \theta\right)^{2}}{n}} \\
& s_{d x}=\sqrt{\frac{\sum_{i=1}^{n}\left(\left(x_{i}-\bar{x}\right) \cos \theta-\left(y_{i}-\bar{y}\right) \sin \theta\right)^{2}}{n}} \\
& s_{d x}=\sqrt{\frac{\sum_{i=1}^{n}\left(\left(\left(x_{i}-\bar{x}\right) \cos \theta\right)^{2}-2\left(x_{i}-\bar{x}\right) \cos \theta\left(y_{i}-\bar{y}\right) \sin \theta+\left(\left(y_{i}-\bar{y}\right) \sin \theta\right)^{2}\right)}{n}}, \\
& s_{d x}=\sqrt{\frac{\cos ^{2} \theta \sum_{i=1}^{n}\left(x_{i}-\bar{x}\right)^{2}-2 \cos \theta \sin \theta \sum_{i=1}^{n}\left(\left(x_{i}-\bar{x}\right)\left(y_{i}-\bar{y}\right)\right)+\sin ^{2} \theta \sum_{i=1}^{n}\left(y_{i}-\bar{y}\right)^{2}}{n}} \\
& s_{d x}=\sqrt{\frac{\cos ^{2} \theta \sum_{i=1}^{n}\left(x^{2}-2 x \bar{x}+\bar{x}^{2}\right)-2 \cos \theta \sin \theta \sum_{i=1}^{n}(x y-\bar{x} y-x \bar{y}+\bar{x} \bar{y})+\sin ^{2} \theta \sum_{i=1}^{n}\left(y^{2}-2 y \bar{y}+\bar{y}^{2}\right)}{n}} \\
& s_{d x}=\sqrt{\frac{\cos ^{2} \theta \sum_{i=1}^{n}\left(x^{2}\right)}{n}-\frac{2 \bar{x} \cos ^{2} \theta \sum_{i=1}^{n}(x)}{n}+\frac{\cos ^{2} \theta \sum_{i=1}^{n}\left(\bar{x}^{2}\right)}{n}} \\
& -\frac{2 \cos \theta \sin \theta \sum_{i=1}^{n}(x y-\bar{x} y-x \bar{y}+\bar{x} \bar{y})+\sin ^{2} \theta \sum_{i=1}^{n}\left(y^{2}-2 y \bar{y}+\bar{y}^{2}\right)}{n} \\
& s_{d x}=\sqrt{\frac{\cos ^{2} \theta \sum_{i=1}^{n}\left(x^{2}\right)}{n}-2 \bar{x}^{2} \cos ^{2} \theta+\bar{x}^{2} \cos ^{2} \theta}-\frac{2 \cos \theta \sin \theta \sum_{i=1}^{n}(x y-\bar{x} y-x \bar{y}+\bar{x} \bar{y})+\sin ^{2} \theta \sum_{i=1}^{n}\left(y^{2}-2 y \bar{y}+\bar{y}^{2}\right)}{n} \\
& s_{d x}=\sqrt{\frac{\cos ^{2} \theta \sum_{i=1}^{n}\left(x^{2}\right)}{n}-\bar{x}^{2} \cos ^{2} \theta-\frac{2 \cos \theta \sin \theta \sum_{i=1}^{n}(x y-\bar{x} y-x \bar{y}+\bar{x} \bar{y})}{n}+\frac{\sin ^{2} \theta \sum_{i=1}^{n}\left(y^{2}\right)}{n}}-\frac{2 \bar{y} \sin ^{2} \theta \sum_{i=1}^{n}(y)}{n} \\
& +\frac{\sin ^{2} \theta \sum_{i=1}^{n}\left(\bar{y}^{2}\right)}{n} \\
& s_{d x}=\sqrt{\frac{\cos ^{2} \theta \sum_{i=1}^{n}\left(x^{2}\right)}{n}-\bar{x}^{2} \cos ^{2} \theta-\frac{2 \cos \theta \sin \theta \sum_{i=1}^{n}(x y-\bar{x} y-x \bar{y}+\bar{x} \bar{y})}{n}+\frac{\sin ^{2} \theta \sum_{i=1}^{n}\left(y^{2}\right)}{n}}-\bar{y}^{2} \sin ^{2} \theta \\
& s_{d x}=\sqrt{\frac{\cos ^{2} \theta \sum_{i=1}^{n}\left(x^{2}\right)}{n}-\bar{x}^{2} \cos ^{2} \theta}-\frac{2 \cos \theta \sin \theta\left(\sum_{i=1}^{n}(x y)-\sum_{i=1}^{n}(\bar{x} y)-\sum_{i=1}^{n}(x \bar{y})+\sum_{i=1}^{n}(\bar{x} \bar{y})\right)}{n}+\frac{\sin ^{2} \theta \sum_{i=1}^{n}\left(y^{2}\right)}{n} \\
& s_{d x}=\sqrt{\frac{\cos ^{2} \theta \sum_{i=1}^{n}\left(x^{2}\right)}{n}-\bar{x}^{2} \cos ^{2} \theta-\frac{2 \cos \theta \sin \theta \sum_{i=1}^{n}(x y)}{\mathrm{n}}+\frac{2 \cos \theta \sin \theta \bar{x} \sum_{i=1}^{n}(y)}{\mathrm{n}}}+\frac{2 \cos \theta \sin \theta \bar{y} \sum_{i=1}^{n}(x)}{\mathrm{n}} \\
& -\frac{n \bar{x} \bar{y} 2 \cos \theta \sin \theta}{\mathrm{n}}+\frac{\sin ^{2} \theta \sum_{i=1}^{n}\left(y^{2}\right)}{n}-\bar{y}^{2} \sin ^{2} \theta \\
& s_{d x}=\sqrt{\frac{\cos ^{2} \theta \sum_{i=1}^{n}\left(x^{2}\right)}{n}-\bar{x}^{2} \cos ^{2} \theta-\frac{2 \cos \theta \sin \theta \sum_{i=1}^{n}(x y)}{\mathrm{n}}+2 \cos \theta \sin \theta \bar{x} \bar{y}+2 \cos \theta \sin \theta \bar{y} \bar{x}}-2 \cos \theta \sin \theta \bar{x} \bar{y} \\
& +\frac{\sin ^{2} \theta \sum_{i=1}^{n}\left(y^{2}\right)}{n}-\bar{y}^{2} \sin ^{2} \theta \\
& s_{d x}=\sqrt{\frac{\cos ^{2} \theta \sum_{i=1}^{n}\left(x^{2}\right)}{n}-\bar{x}^{2} \cos ^{2} \theta-\frac{2 \cos \theta \sin \theta \sum_{i=1}^{n}(x y)}{\mathrm{n}}+2 \cos \theta \sin \theta \bar{x} \bar{y}+\frac{\sin ^{2} \theta \sum_{i=1}^{n}\left(y^{2}\right)}{n}}-\bar{y}^{2} \sin ^{2} \theta
\end{aligned}
$$

\section{Similarly:}




$$
\begin{aligned}
& s_{d y}=\sqrt{\frac{\sum_{i=1}^{n}\left(\left(x_{i}-\bar{x}\right) \sin \theta-\left(y_{i}-\bar{y}\right) \cos \theta\right)^{2}}{n}} \\
& s_{d y}=\sqrt{\frac{\sin ^{2} \theta \sum_{i=1}^{n}\left(x^{2}\right)}{n}-\bar{x}^{2} \sin ^{2} \theta-\frac{2 \cos \theta \sin \theta \sum_{i=1}^{n}(x y)}{\mathrm{n}}-2 \cos \theta \sin \theta \bar{x} \bar{y}+\frac{\cos ^{2} \theta \sum_{i=1}^{n}\left(y^{2}\right)}{n}}-\bar{y}^{2} \cos ^{2} \theta
\end{aligned}
$$

\section{Rearrangement of Equation 2.15 for single pass calculation}

$A=\sum_{i=1}^{n}(x-\bar{x})^{2}-\sum_{i=1}^{n}(y-\bar{y})^{2}$

$A=\sum_{i=1}^{n}\left(x^{2}\right)-2 \bar{x} \sum_{i=1}^{n}(x)+n \bar{x}^{2}-\sum_{i=1}^{n}\left(y^{2}\right)+2 \bar{y} \sum_{i=1}^{n}(y)-n \bar{y}^{2}$

$$
\begin{aligned}
& \text { Rearrangement of Equation } 2.16 \text { for single pass calculation } \\
& B=\sqrt{(A)^{2}+4 \sum_{i=1}^{n}((x-\bar{x})(y-\bar{y}))^{2}} \\
& B=\sqrt{(A)^{2}+4\left(\sum_{i=1}^{n}\left(\left(x^{2}-2 x \bar{x}+\bar{x}^{2}\right)\left(y^{2}-2 y \bar{y}+\bar{y}^{2}\right)\right)\right)} \\
& B=\sqrt{(A)^{2}+4\left(\sum_{i=1}^{n}\left(x^{2} y^{2}-2 x^{2} y \bar{y}+x^{2} \bar{y}^{2}-2 x \bar{x} y^{2}+22 x \bar{x} y \bar{y}-2 x \bar{x} \bar{y}^{2}+\bar{x}^{2} y^{2}-2 \bar{x}^{2} y \bar{y}+\bar{x}^{2} \bar{y}^{2}\right)\right)} \\
& B=\sqrt{(A)^{2}+4\left(\sum_{i=1}^{n} x^{2} y^{2}-2 \bar{y} \sum_{i=1}^{n} x^{2} y+\bar{y}^{2} \sum_{i=1}^{n} x^{2}-2 \bar{x} \sum_{i=1}^{n} x y^{2}+4 \bar{x} \bar{y} \sum_{i=1}^{n} x y-2 \bar{x} \bar{y}^{2} \sum_{i=1}^{n} x+\bar{x}^{2} \sum_{i=1}^{n} y^{2}-2 \bar{x}^{2} \bar{y} \sum_{i=1}^{n} y+n \bar{x}^{2} \bar{y}^{2}\right)}
\end{aligned}
$$

Rearrangement of Equation 2.17 for single pass calculation

$C=2 \sum_{i=1}^{n}((x-\bar{x})(y-\bar{y}))$

$C=2\left(\sum_{i=1}^{n}(x y-\bar{x} y-x \bar{y}+\bar{x} \bar{y})\right)$

$C=2\left(\sum_{i=1}^{n}(x y)-\sum_{i=1}^{n}(\bar{x} y)-\sum_{i=1}^{n}(x \bar{y})+\sum_{i=1}^{n}(\bar{x} \bar{y})\right)$

$C=2\left(\sum_{i=1}^{n}(x y)-\bar{x} \sum_{i=1}^{n}(y)-\bar{y} \sum_{i=1}^{n}(x)+n \bar{x} \bar{y}\right)$ 